\title{
A Compendium of
}

NHTSA's Pedestrian and Bicyclist Traffic Safety Research Projects 1969-2007

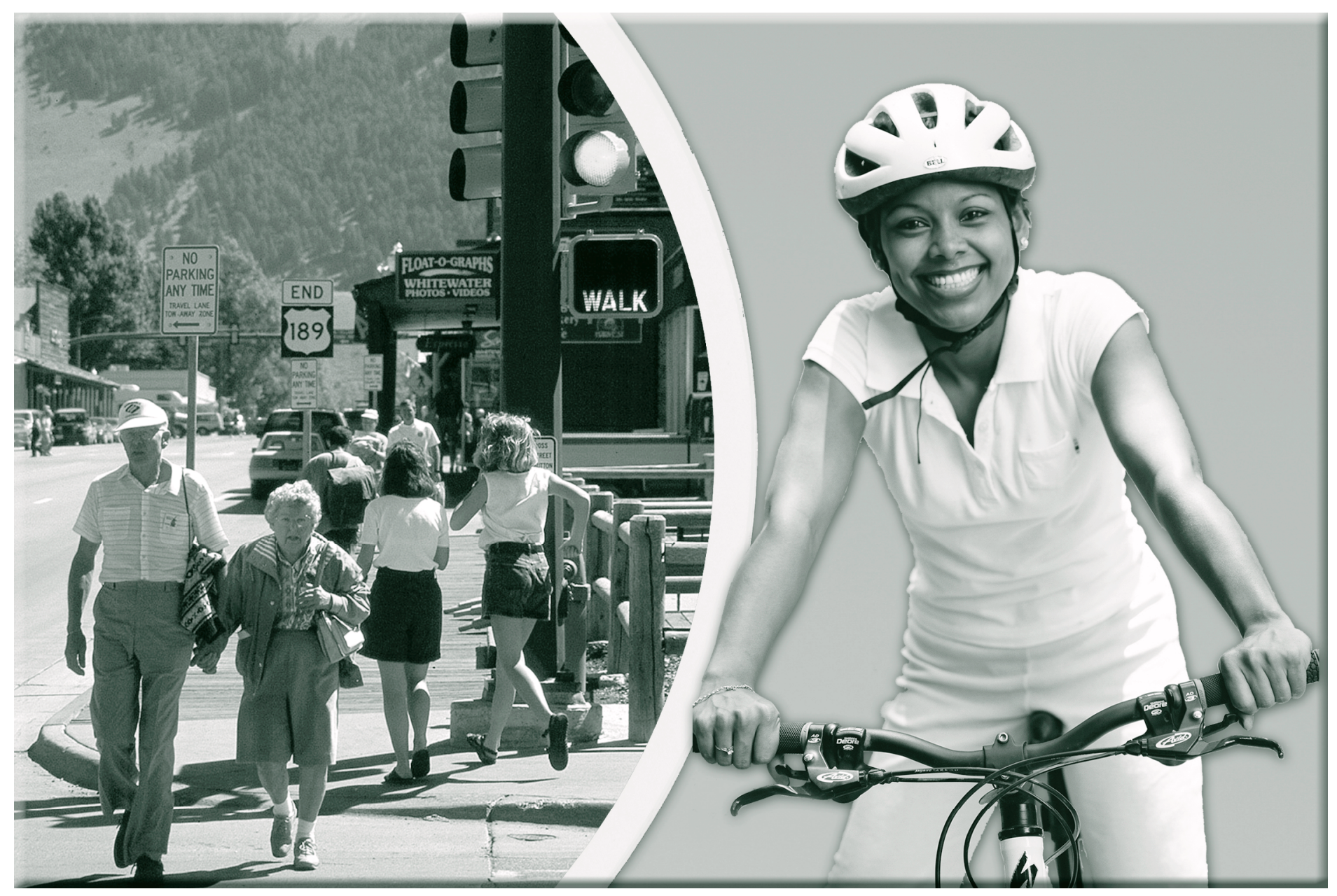


This publication is distributed by the U.S. Department of Transportation, National Highway Traffic Safety Administration, in the interest of information exchange. The opinions, findings, and conclusions expressed in this publication are those of the authors and not necessarily those of the Department of Transportation or the National Highway Traffic Safety Administration. The United States Government assumes no liability for its contents or use thereof. If trade or manufacturers' names or products are mentioned, it is because they are considered essential to the object of the publication and should not be construed as an endorsement. The United States Government does not endorse products or manufacturers.

Cover photograph of pedestrians by Dan Burden, www.pedbikeimages.org 
Technical Report Documentation Page

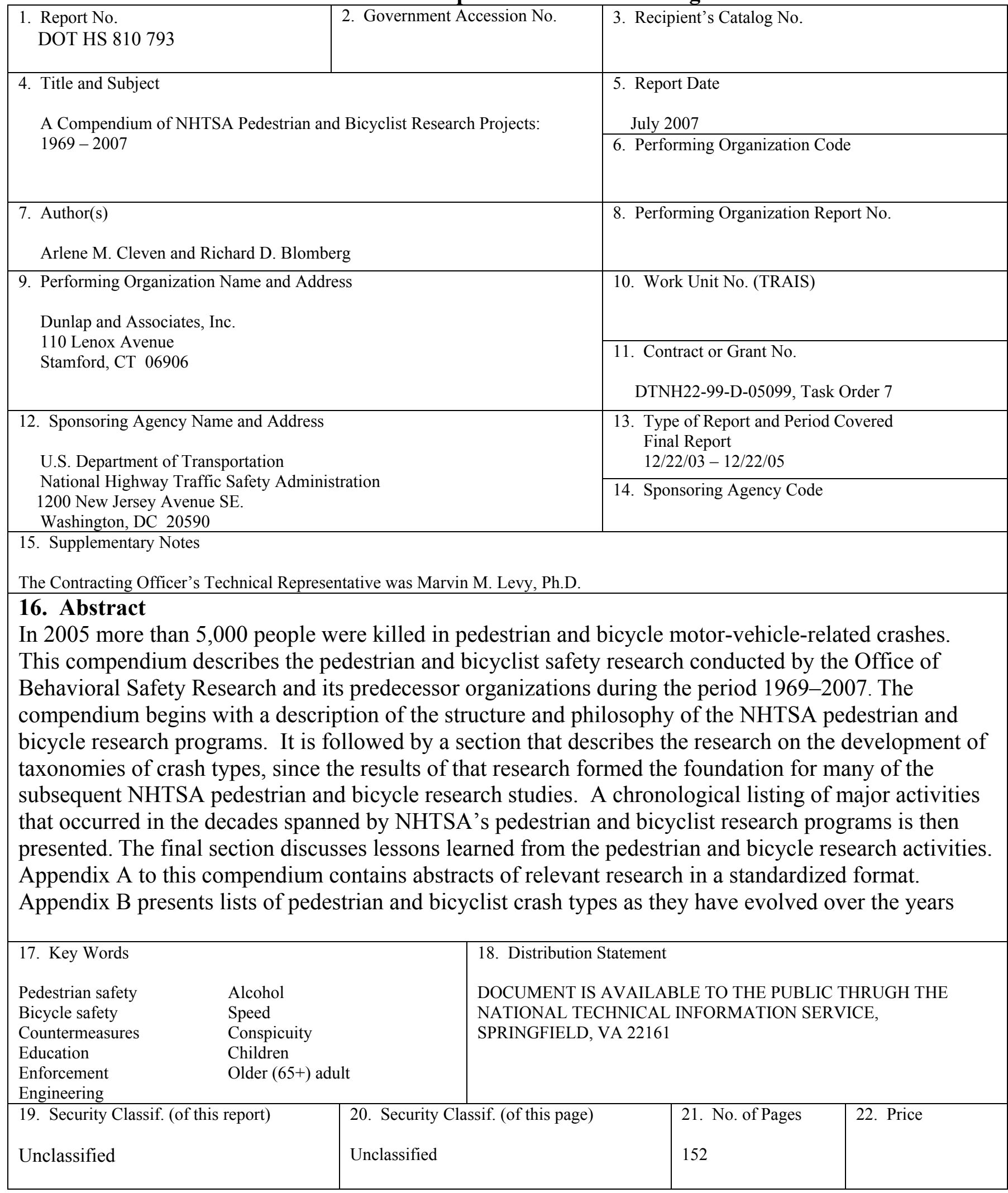

Form DOT F 1700.7 (8-72) 


\section{ACKNOWLEDGMENT}

This report represents a revision, update, and consolidation of two draft documents originally prepared for the National Highway Traffic Safety Administration by Dr. Alfred J. Farina of Safety Consulting Services. 


\section{TABLE OF CONTENTS}

INTRODUCTION …………

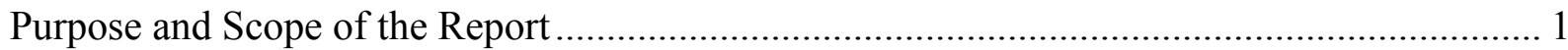

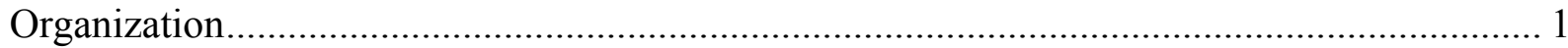

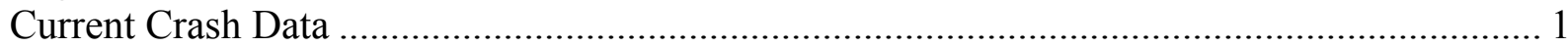

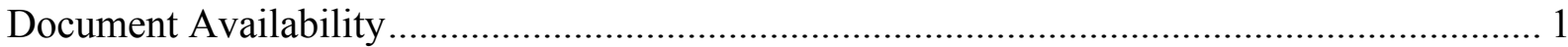

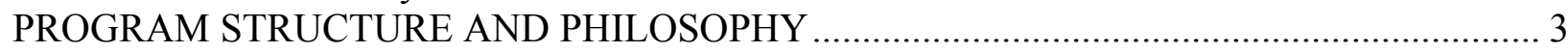

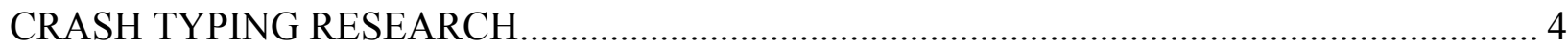

A Behavioral Approach to Pedestrian Safety ………........................................................ 4

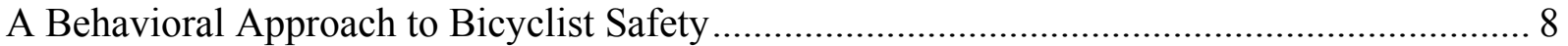

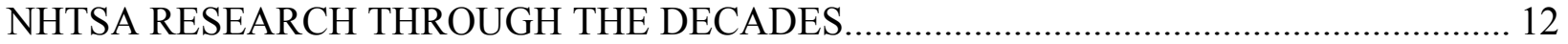

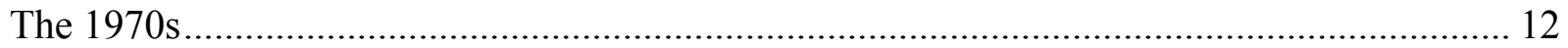

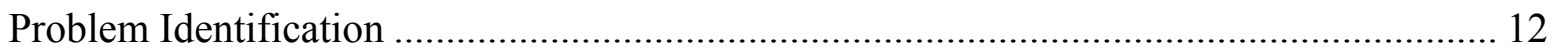

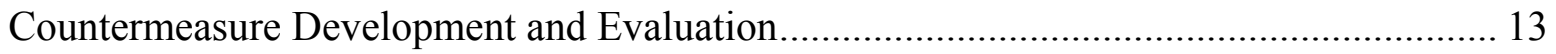

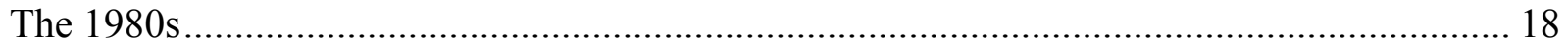

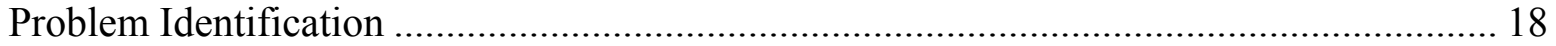

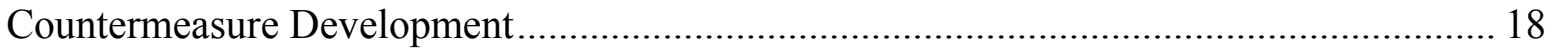

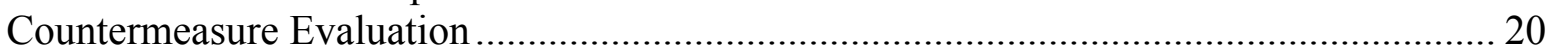

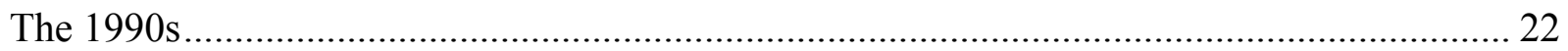

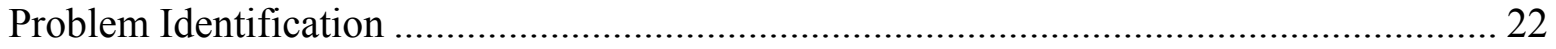

Countermeasure Development.................................................................................... 23

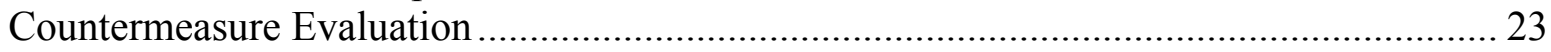

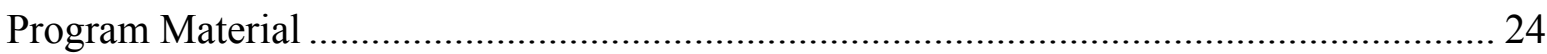

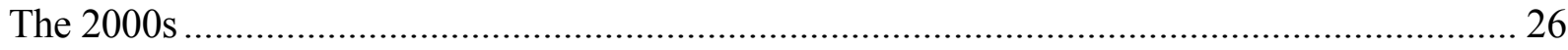

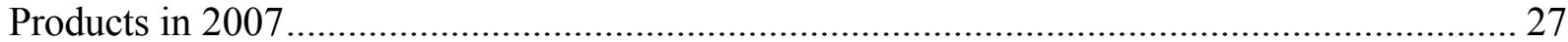

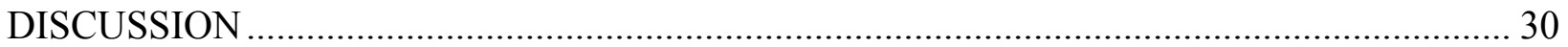

Problem Identification ................................................................................................... 30

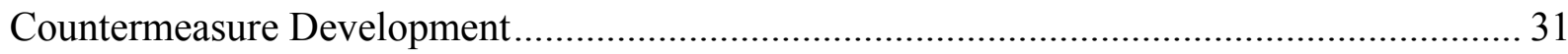

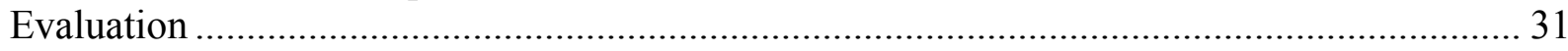

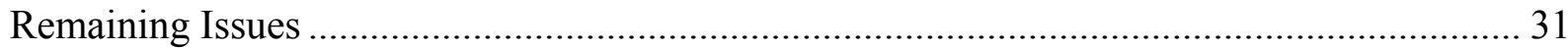

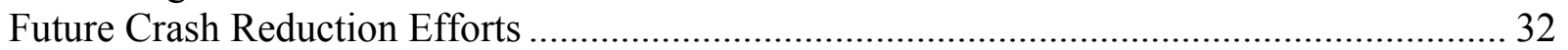

APPENDIX A - SUMMARIES OF NHTSA PEDESTRIAN AND BICYCLIST SAFETY

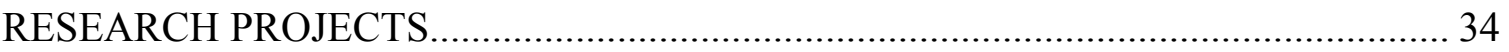

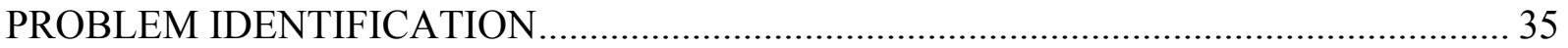

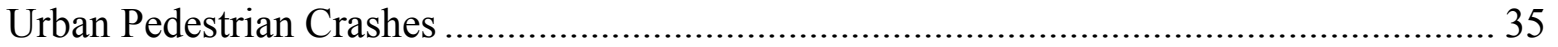

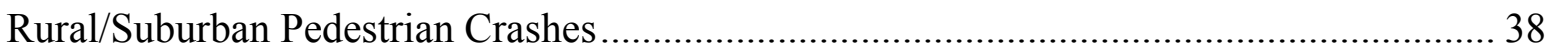

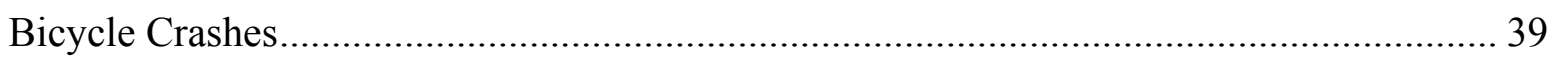

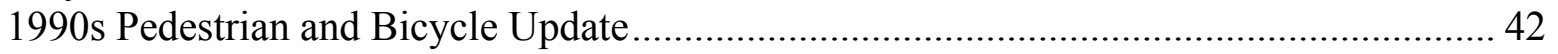

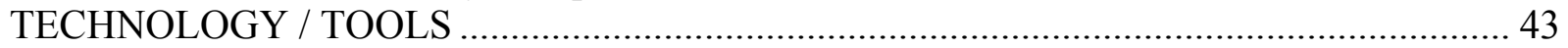

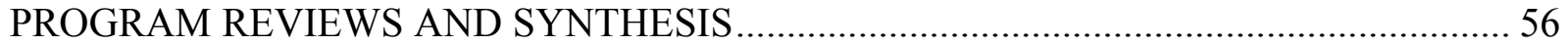

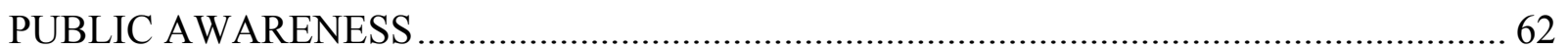

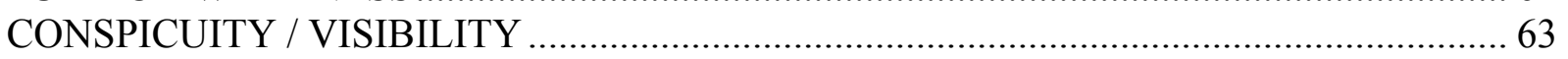

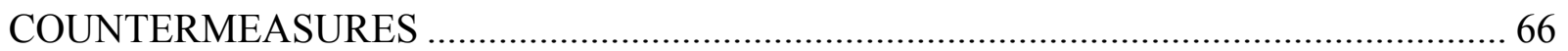

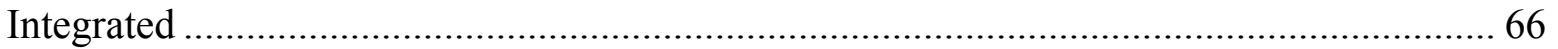

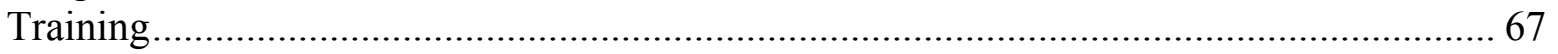

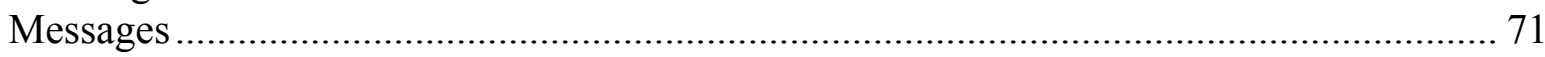

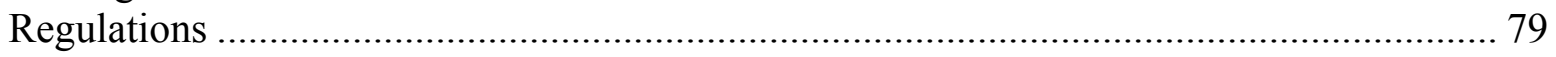




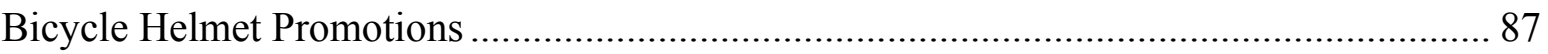

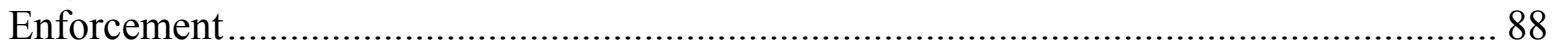

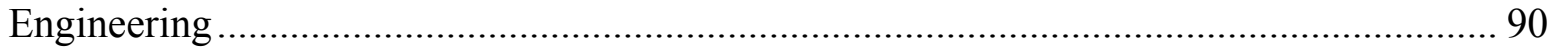

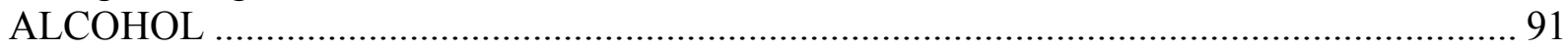

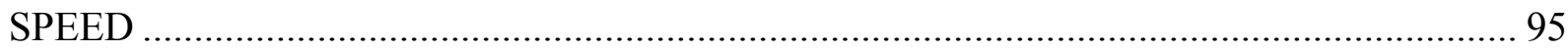

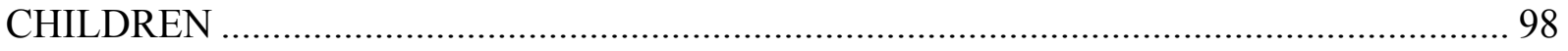

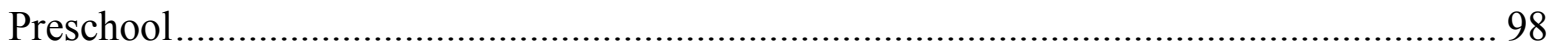

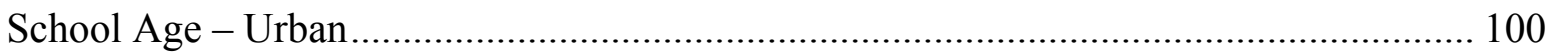

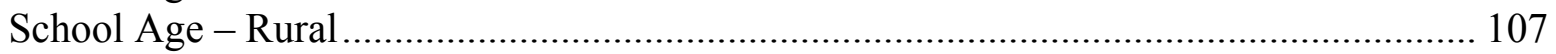

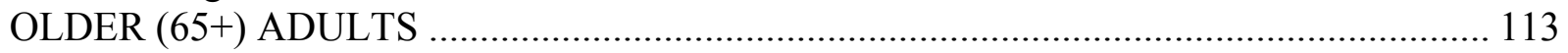

APPENDIX B - PEDESTRIAN AND BICYCLIST CRASH TYPES ..................................... 115

Snyder and Knoblauch (1971) Pedestrian Crash Types ...................................................... 115

NHTSA (1983) Pedestrian Crash Types......................................................................... 117

Harkey et al. (2000) PBCAT Pedestrian Crash Types.......................................................... 120

Cross and Fisher (1977) Bicyclist Crash Types................................................................. 124

Harkey et al. (2000) PBCAT Bicyclist Crash Types ........................................................ 126

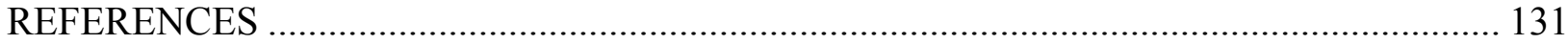

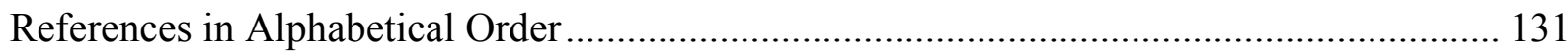

References by Ascending Date of Publication.................................................................. 138

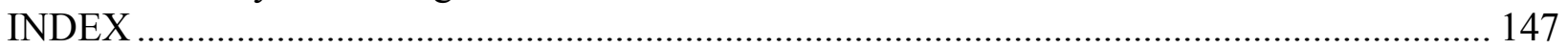

\section{List of Figures}

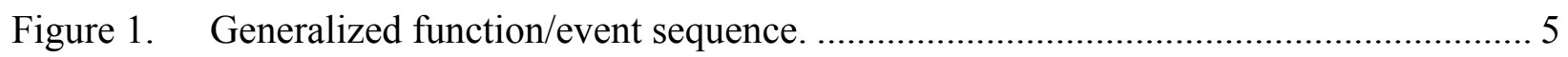

Figure 2. Conceptual model of the crash generation process .................................................... 9 


\section{INTRODUCTION}

\section{Purpose and Scope of the Report}

The purpose of this report is to provide an overview of the National Highway Traffic Safety Administration's pedestrian and bicycle safety research programs. In addition to describing the process employed and the lessons learned, it also provides a chronological description of the various programs and synopses of relevant projects.

This document covers the pedestrian and bicyclist safety research produced by the Office of Behavioral Safety Research and its predecessor organizations from the inception of the program in 1969 through the early 21st century. Some work done by the Federal Highway Administration is also included because of its relevance to the NHTSA program.

\section{Organization}

This compendium begins with a description of the structure and philosophy of the NHTSA pedestrian and bicycle research programs. It is followed by a section that describes the research on the development of taxonomies of crash types ${ }^{1}$ since the results of that research formed the foundation for most of the subsequent NHTSA pedestrian and bicycle research studies. A chronological listing of major activities that occurred in the decades spanned by NHTSA's pedestrian and bicyclist research programs is then presented. The final section discusses lessons learned for future pedestrian and bicycle research. Appendix A to this compendium contains abstracts of relevant research in a standardized format. Appendix B presents lists of pedestrian and bicyclist crash types as they have evolved over the years. A reference section lists documents identified in the compendium. Finally, an index of authors and principal subject matter is provided to assist the user in identifying research of interest.

\section{Current Crash Data}

NHTSA's National Center for Statistics and Analysis publishes annual summaries of nationwide pedestrian and bicyclist crash data. The latest of these Traffic Safety Facts publications can be found on the NHTSA Web site ${ }^{2}$ by following the "Traffic Safety" tab and clicking on the topic of interest - pedestrians or bicyclists. Data presented here was current at the time of writing.

\section{Document Availability}

Many of the reports described in this document can be purchased in paper or other formats from the National Technical Information Service (NTIS) at the following address:

\footnotetext{
${ }^{1}$ Crash types are pedestrian or bicycle collisions with motor vehicles with specific driver and/or pedestrian behavioral causes and environmental circumstances. For example, a Residential Driveway Rideout bicycle/motor vehicle crash involves a bicyclist who appears suddenly in the roadway from a residential driveway without searching for motor vehicles and is not seen until too late by the striking motor vehicle driver.

${ }^{2}$ www.nhtsa.dot.gov
} 
National Technical Information Service

5285 Port Royal Road

Springfield, VA 22161

703-605-6000

800-553-NTIS

For documents available through NTIS, the NTIS order numbers (which start with the letters PB) are included in the references section of this report. Most documents available from NTIS are reproduced from their electronic archive and are not original stock.

Some of the recent reports described in the document may be available for download on NHTSA's Web site (http://www.nhtsa.dot.gov).

Contact may also be made directly with NHTSA's Office of Behavioral Safety Research at the following address:

Pedestrian/Bicyclist Research Program Manager

Code NTI-131

1200 New Jersey Avenue, SE.

Washington, DC 20590

202-366-4892 


\section{PROGRAM STRUCTURE AND PHILOSOPHY}

Pedestrian and bicycle crashes have likely been studied for as long as there has been an interest in traffic safety. Prior to the 1970s, however, the general focus was on counting events and admonishing people, both young and old, to "be safe." Little effort was directed at a systematic analysis and understanding of crash causes and the means to prevent them. The countermeasure efforts that did exist tended to be focused on the entire population of crashes or major demographic subsets of them such as those involving school-aged children.

In the late 1960s, a research decision by NHTSA fundamentally altered the way in which pedestrian and bicycle crashes were analyzed and addressed by countermeasures. The decision involved trying to disaggregate all pedestrian crashes into specific crash types with similar behavioral causes. The resulting study by Snyder and Knoblauch (1971) has been the cornerstone of NHTSA's pedestrian research program ever since. Between the publication of their report and the present, numerous studies have been conducted using crash types as a focus. Many efforts were directed at the development of specific interventions for the identified types. Other studies dealt with uncovering a better understanding of the crash types themselves including any differences as a function of locale (urban, suburban, rural) or any changes over time. Emulating the work conducted in the pedestrian safety area, NHTSA then repeated the crash type identification process for bicycle/motor vehicle crashes in a landmark study by Cross and Fisher (1977).

The generation of crash taxonomies was a productive first step for the NHTSA pedestrian and bicycle research program. In addition, the decision to base the taxonomies on the behaviors of the crash participants - pedestrians, bicyclists and drivers - proved critical in making the resulting crash types particularly useful as a basis for countermeasures. Simply, by focusing on the defined types, countermeasures were in essence dealing directly with the underlying behavioral errors that cause them. This is true regardless of whether the countermeasure itself consisted of engineering, education or enforcement components or a combination thereof.

Once crash types were defined, the NHTSA research program turned to the development and evaluation of countermeasures based on public information and education, training and enforcement, areas for which NHTSA is responsible. FHWA focused on engineering approaches to crash prevention. Together, these initial forays into prevention efforts produced both useful products and a better understanding of the basic behaviors needed to avoid crashes. Perhaps the most important and fundamental of the principles identified was the preeminent importance of the search function for crash avoidance. The benefit of a left-right-left search pattern for everyone in the traffic environment was identified as a critical basic behavior.

Because of the overarching importance of the Snyder and Knoblauch (1971) and Cross and Fisher (1977) studies to understanding the NHTSA program and, in fact, the entire study of pedestrian and bicycle crashes, detailed descriptions of these crash typing research efforts are presented in the next section. 


\section{CRASH TYPING RESEARCH}

Prior to the landmark crash type identification studies of Snyder and Knoblauch (1971) and Cross and Fisher (1977), pedestrian and bicycle crash taxonomies were largely based on the demographics of the event: the victim's personal information (age, gender, etc.), crash location, time of day, day of week, weather conditions, road geometry, injuries incurred, and other data typically contained on a police accident report. While informative and useful, this type of classification system is not very effective in revealing why crashes occur and how to prevent them.

In order to focus countermeasure efforts, a different type of classification system was required - one that is focused on the behavior of the participants of the crash. Why did the pedestrian step in front of the car? Why did the driver hit the bicyclist while making a turn? Addressing the why and how of the pedestrian or bicyclist crash required collecting information on the behavior of all involved and relating that information to the demographic and environmental data. To fill this gap, it was necessary to examine pedestrian and bicyclist crashes from a behavioral point of view.

In 1969, the newly formed NHTSA sponsored what has since become a seminal study in pedestrian safety. The study - conducted by Snyder and Knoblauch (1971) - concentrated more closely than ever before on behavioral factors - the how and why of pedestrian crashes. Its focus was to understand crash causation and to create a knowledge base that could be used to develop behavioral solutions for preventing the specific types of crashes identified. The rationale was that the detailed information provided by the behaviorally-based crash types would lead to more effective and efficient interventions regardless of the intervention mode.

This study by Cross and Fisher (1977) used the same basic crash causation model pioneered by Snyder and Knoblauch (1971) and added some additional refinements in data collection and analysis. Both of these studies are described in detail below.

\section{A Behavioral Approach to Pedestrian Safety}

Snyder and Knoblauch (1971) developed a behavioral model of the crash situation as a guide for their research (see Figure 1). This function/event sequence was also used by Cross and Fisher (1977) for bicyclists and motor vehicle drivers. The model consists of the key behaviors or functions in the sequence leading to a crash. The premise is that the successful completion of the sequence by either party results in crash avoidance. Both parties must fail to complete the sequence successfully in order for a crash to occur. The critical functions in the sequence are: 
DRIVER AND VEHICLE

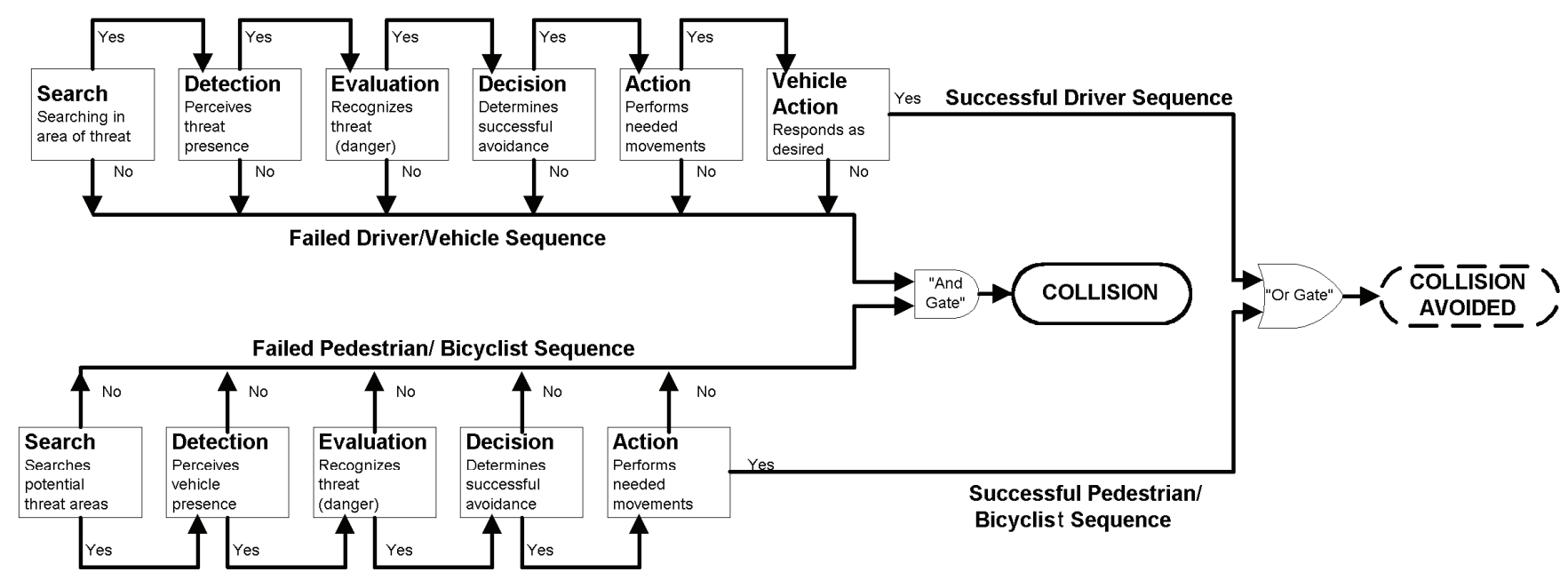

PEDESTRIAN/ BICYCLIST

Adapted from Snyder and Knoblauch (1971)

Figure 1. Generalized function/event sequence.

Search: $\quad$ Both driver and pedestrian/bicyclist scan their environment for potential hazards.

Detection: $\quad$ Each sees the other.

Evaluation: $\quad$ Each recognizes the threat of a collision and the need for action to avoid it.

Decision: $\quad$ Each determines what action to take to avoid a collision.

Action: $\quad$ Either pedestrian/bicyclist or driver or both successfully perform(s) the appropriate action.

Vehicle response: A factor for a motor vehicle or bicycle driver is the response of the vehicle to the action taken.

The sequence begins when the driver and pedestrian/bicyclist commence on a collision course making a crash/incident likely unless an avoidance maneuver is taken. If either party to a potential crash performs the sequence successfully, that crash is avoided. Thus, by definition, both parties must suffer a failure of their portion of the function/event sequence for a crash to occur. This failure, however, is not the same as culpability since circumstances surrounding the performance of the function/event sequence that are not under the control of one or both of the parties may make it impossible to complete the sequence successfully. For example, a pedestrian may fail to see a car at night because the driver forgot to turn on the headlights or a pedestrian 
may be effectively camouflaged by wearing dark clothing at night or clothing that blends into the background during the day.

It is also important to note in Figure 1 that this function/event sequence is a dependent sequential process. When a participant fails to complete a function successfully (a "No" exit from any of the functions in Figure 1), all subsequent functions must also fail. For example, if a motorist searches but fails to detect a pedestrian, the motorist cannot properly evaluate the situation, make an appropriate decision nor take the proper action. In this example, avoidance of a crash will therefore depend solely on the successful performance of the sequence by the pedestrian. In the event the pedestrian also does not detect the potential striking vehicle, a collision will occur (unless external intervention occurs - such as by a parent intervening in the crossing behavior of a child.). Since "Search" is the first function in the sequence, it becomes controlling and most important in the prevention of a crash. The failure to search at all or the performance of an ineffective search is implicated in the vast majority of pedestrian crashes, especially those involving young children.

This model was elaborated upon to include environmental considerations. From it, the researchers generated the questions to be asked about the crash. They investigated approximately 2,000 pedestrian crashes in 13 large cities across the country. About 500 items (demographic, environmental, and behavioral) were obtained for each crash through interviews with the victims, witnesses, drivers, and the police, and through visits made to the crash site.

The behavioral model also guided how the data were analyzed. There were found to be predisposing factors - things that set the stage for the crash or made the crash more likely to occur. These predisposing factors included characteristics or environmental conditions such as parked cars (they screen the pedestrian and the driver from seeing each other), and human factors such as alcohol (it degrades many of the pedestrian's processes). A single event can have one or more of these factors operating to predispose or "tilt" the situation toward a crash.

Then there were the human errors or failures in the function/event sequence. These were seen as precipitating events to highlight their more immediate relationship to the crash. They could be errors of omission, or poor performance, or delays in execution/timing. They were more directly related to the crash itself, often triggering it. Poor search behavior, or errors made in selecting a "course" (where to enter the street) are high-frequency examples.

The approximately 2,000 crash cases were examined in terms of predisposing factors, precipitating events, and target groups (victims such as young children or the elderly). It was here that Snyder and Knoblauch (1971) made a significant decision. They believed:

...that similarities and differences among the population of pedestrian accidents are such that a finite number of different types can be identified, each of which will be amenable to different (but perhaps overlapping) countermeasures. In other words, in order to identify countermeasures, pedestrian accidents should be broken down into different types primarily on the basis of cause. Each such grouping is designated as a 'causal type.' (1971, Vol. I, page 3-7) 
The Snyder and Knoblauch (1971) study yielded about 30 different crash types, but many were low-frequency events. Five of the crash types accounted for more than half the total number of crashes in the sample. These crash types included:

- Dart-out (first half) - where the (typically young) pedestrian appears suddenly midblock, often from between parked cars, presents a limited exposure time to the driver and is struck less than half way across the roadway.

- Dart-out (second half) - similar to the Dart-out (first half) except the pedestrian is struck after crossing half or more of the roadway.

- Intersection dash - where the pedestrian presents a short time exposure to the driver at an intersection either because the pedestrian runs across the intersection, is blocked from view or is not expected to be crossing by the driver, and is struck.

- Multiple threat - where a vehicle stops for a crossing pedestrian and, in so doing, blocks the pedestrian from the view of the driver in a second car that is overtaking the first car (includes intersection and midblock situations).

- Vehicle turn/merge - where the pedestrian is usually not seen by the driver who is concentrating on turning into or merging with traffic. Both the driver and the pedestrian workloads are likely increased by the complexity of the crossing situation at an intersection. For example, the pedestrian has to deal with turning vehicles and vehicles driving straight through the intersection and possibly traffic signals. The driver must find gaps in traffic in the first and second half of the roadway before merging.

Other crash types identified by Snyder and Knoblauch (1971) with significant frequencies that became the focus of initial countermeasure efforts included:

- Commercial-bus-related - where a pedestrian crosses in front of a stopped bus, is screened by the bus from the view of an overtaking driver, and is struck stepping out into the overtaking driver's lane.

- Vendor/ice cream truck - where a young child is struck by a passing vehicle while going to or from a vending vehicle that screens the child from an overtaking driver's view.

- Backing vehicle - where the pedestrian is struck by a vehicle that is backing up in the street, out of a driveway, or in a parking lot.

In the years that followed the Snyder and Knoblauch (1971) study, other investigators added several more crash types to the list of "major types," thus further raising the percent of crashes covered by a relatively small set. Thereafter, some crash types were merged because they could be treated by the same countermeasures. A case in point is the "collapsing" of the dart-out first and second half types into a single type because the countermeasures for both types were the same. Nevertheless, the basic premises established by the Snyder and Knoblauch (1971) study and their benefit for research and crash investigation have proved enduring. 


\section{A Behavioral Approach to Bicyclist Safety}

In 1974, the mounting toll of bicycle/motor-vehicle fatalities led NHTSA to initiate research on the causes of these crashes and ways of preventing them. Fatalities in that year reached nearly 1,000 bicyclists, and that number did not include those bicyclists who were injured or killed in single-vehicle crashes, i.e., those not involving a motor vehicle. Bicycling was increasing in popularity, there being several years in which large numbers of bicycles were sold.

In conducting the study, the task facing the researchers (Cross and Fisher, 1977) involved examining crashes involving bicycles and motor vehicles - and classify these disparate events into mutually exclusive categories for which specific countermeasures can be tailored.

Their first step was to conceive of a general model to describe the crash situation. In doing so, they were significantly influenced by the work of Snyder and Knoblauch (1971) in the pedestrian area and, to a lesser extent, by the work of Baker (1961), Baker and Ross (1961), Fell (1974), McGlade and Laws (1962), and Perchonok (1975). The models served to structure or organize the flow of information, events, outcomes, processes, errors, etc., that characterize the crash. The investigators began the task by defining individual components and hypothesizing how they interrelate to lead to crashes. The Cross and Fisher conceptualization has many parts and is best understood by reference to Volume I of their final report (Cross \& Fisher, 1977). Borrowing heavily from that document here, a brief overview begins with defining the cause of a crash in terms of the full range of factors that could contribute to it. As quoted from Cross and Fisher (1977), these included:

- Operator factors - Operator factors include operator conditions that were subnormal or atypical at the time of the accident and that contributed directly or indirectly to the accident. Operator factors also include specific behavioral acts performed by the operator that are considered subnormal or atypical and that had a contributory effect.

- Vehicle factors - Vehicle factors include vehicle failures and vehicle design features that contributed directly or indirectly to the crash.

- Environmental factors - Environmental factors include weather conditions, lighting conditions, roadway conditions, traffic conditions, and any other environmental object or condition that contributed to the accident. (Cross \& Fisher, 1977, vol I, page 30)

In this context, "subnormal" means that the operator performed the assigned task but at a lower level of performance than would be expected for the normal range of operators. "Atypical" indicates that the operator performed a task that was unusual or inappropriate for similar types of operators.

The three classes of factors became the foundation of a conceptual model of the crash generation process shown in Figure 2. Additional features of that model-function failures, critical actions, and terminal events (crash) — bring it to completion. 


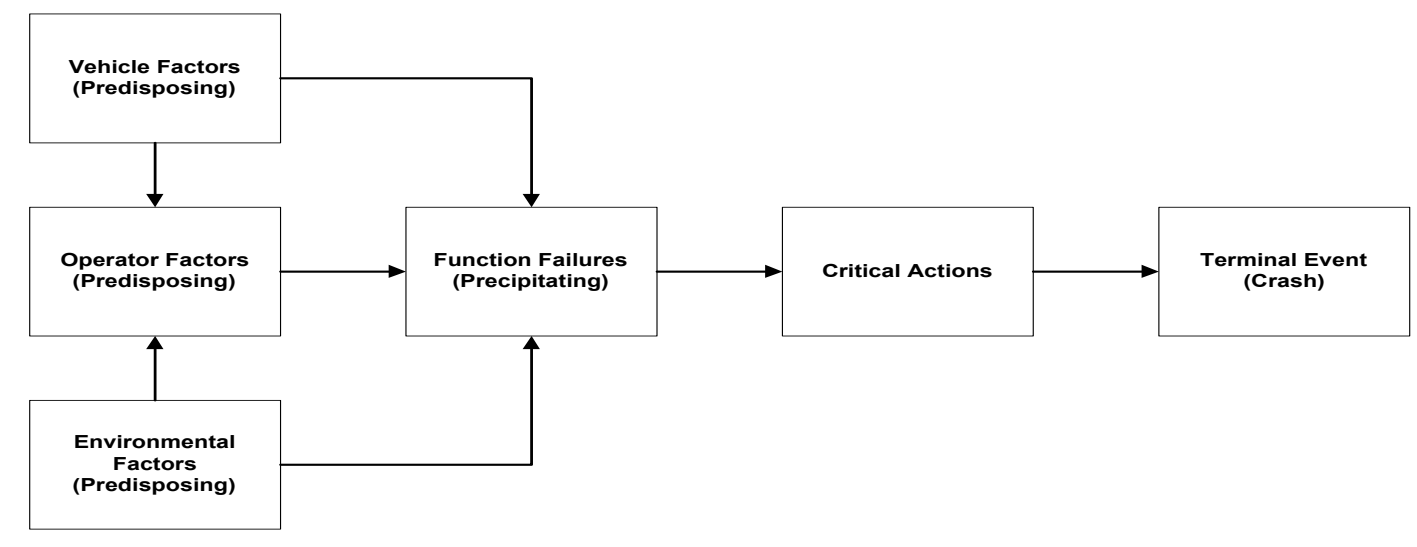

Figure 2. Conceptual model of the crash generation process

(Adapted from Cross and Fisher, 1977, vol I, page 32)

Components of the model are described below:

- Critical actions - Critical actions refer to the vehicles' (motor vehicle and bicycle) actions and movement patterns that led directly to the crash. They constitute the ultimate target for crash countermeasures, and the only criterion for the success of a countermeasure is whether it produces the desired change in the critical actions of at least one of the vehicles. Critical actions cannot meaningfully be described out of context. Many are commonplace actions and motion patterns (turning left, turning right) that are performed every day. A meaningful definition of the critical actions must include a description of the relevant attributes of the roadway and the traffic environment in which the critical actions occurred.

- Function failures - Events that are causally related to the critical actions are characterized as operational failures of the traffic system. That is, one or more elements of the traffic system failed to perform as expected. Operators can fail, vehicles can fail, and certain elements of the environment can fail.

- Terminal event - The terminal event is a crash involving a bicycle and any type of vehicle. Information about the terminal event alone provides no insight about crash causation, but it is needed to assess the consequences of the crash and the potential value of "at-crash" countermeasures.

As with the pedestrian model, the term "predisposing" in Figure 2 is applied to factors that set the stage or lay the groundwork for the crash but do not trigger it. Precipitating, on the other hand, refers to the human errors or function failures more directly related to the crash itself, often triggering it. Existing evidence available during the 1970s indicated that most vehicular crashes are precipitated by an operator (bicyclist/driver) failure. Since it was expected that a very large portion of the function failures for bicycle/motor vehicle crashes would be behavioral, it was considered important that a conceptual framework be developed that would prove useful in identifying and defining the behavioral acts that constitute function failures. 
The behavioral sequence model, developed by Snyder and Knoblauch (1971) for the study of pedestrian crashes, proved to have equal utility for examining bicycle/motor vehicle crashes. Cross and Fisher (1977), however, took a finer grain approach to the safety sequence model by defining three different functional phases: the preparatory phase, the anticipatory phase, and the reactive phase as follows:

- The preparatory phase commences when the operator makes a decision to execute a trip and terminates at the point at which the operator begins the task of selecting a course through the crash area.

- The anticipatory phase commences at the point where the operator begins to perform the tasks required to select a course through the crash area. This phase terminates at the point where the other vehicle (the vehicle with which the operator subsequently collides) first could have been observed if the operator had been looking in the proper direction.

- The reactive phase commences at the point where the other vehicle first becomes observable, and terminates at the collision point.

They were able to identify five "anchor points" within the phases that enabled the investigators to assess the timeliness with which the functions of the behavioral sequence were performed. These anchor points were:

- Collision point - The point at which the vehicles collided; or, if the vehicles did not collide, the point of the first harmful event.

- Point of first evasive action - The point at which the operator first initiated action in an attempt to avoid a collision; or, if a collision was imminent, to reduce the force of impact.

- Point of first alarm - The point at which the operator first recognized that the vehicle was on a collision course with another vehicle, and that a collision would occur if evasive action was not taken by one or both operators.

- Point of first detection - The point at which the presence of the other vehicle was first perceived.

- Point of first observable - The point at which the presence of the other vehicle could first have been detected if the operator had been scanning in the proper direction and had been alert.

The objective of the procedure was to classify a sample of crash cases into mutually exclusive problem types. Cross and Fisher (1977) used a hierarchical system composed of problem classes, types, and subtypes. They explained that:

Problem classes reflect commonality at the most general level. Problem types represent variations of accidents within the same class, and subtypes represent variations of accidents within the same type. Washington, DC: National Highway Traffic Safety Administration. (Cross \& Fisher, 1977, vol I, page 171) 
As Cross and Fisher (1977) noted, the three-level system was developed so that the problem types generally provide the most useful definition of a problem for which specific countermeasures can be tailored. In some cases, however, an entire class or a specific subtype may best serve as a problem definition for the identification of some types of countermeasures.

The Cross and Fisher (1977) study identified seven problem classes and 36 mutually exclusive problem types. Most problem types had two or more subtypes. The term "problem type" as used here is directly comparable to the term "crash type" used in the discussion of pedestrian crashes. As was the case in the pedestrian area, a large percentage of the total bicyclist crashes is accounted for by a small set of problem types. The full set of crash types is included in Appendix B. The seven major problem types were:

- Bicyclist ride-out from a residential driveway - where the bicyclist appears suddenly from a residential driveway, often in front of a parked car and presents a limited exposure time to the driver.

- Bicyclist ride-out from a controlled intersection - where the bicyclist enters an intersection suddenly and presents a limited exposure time to the driver.

- Motorist turn/drive-out in front of bicyclist midblock - where the motor vehicle turns into traffic midblock directly into the path of an oncoming bicyclist.

- Motorist turn/drive-out in front of bicyclist from controlled intersection - where the motor vehicle turns at a controlled intersection directly into the path of an oncoming bicyclist.

- Motorist overtaking/failure to detect bicyclist - where the motorist "rides up the back" of a bicyclist going in the same direction that the motorist failed to detect.

- Bicyclist unexpected left turn/swerve in front of traffic - where a bicyclist who is detected by the motorist makes a sudden turn or swerve into the path of the motorvehicle.

- Motorist unexpected left turn in front of cyclist approaching from straight ahead - where a motorist turns left at a junction and fails to detect a bicyclist proceeding in the opposite direction.

As with pedestrian crash types, bicyclist crash types have been refined over the years, but no fundamental shifts in problem types has been uncovered. The types have formed the foundation for the entire NHTSA research program which has evolved logically and productively in the decades following the original taxonomy studies. 


\section{NHTSA RESEARCH THROUGH THE DECADES}

This section presents an overview of the activities occurring in the decades spanned by NHTSA's pedestrian and bicyclist research programs. The crash type reports described previously for pedestrian (Snyder \& Knoblauch, 1971) and bicyclist crashes (Cross \& Fisher, 1977) were both published in the 1970s and formed the basis for launching numerous countermeasure development efforts as well as research on specific interest areas, such as conspicuity, within pedestrian and bicycle safety.

\section{The 1970s}

The information provided by the crash types (along with the backup supporting data) proved invaluable for three kinds of research:

- Problem identification - field research identifying crash types, target victims, frequencies.

- Countermeasure development - preventive solutions (countermeasures) were developed and tested for their ability to change crash-producing behaviors associated with specific crash types.

- Countermeasure evaluation - promising countermeasures were tested for their ability to reduce specific crash types.

\section{Problem Identification}

Three major problem identification studies were conducted in the 1970s and provided the basic framework for virtually all of the remaining research projects. The three studies dealt with the urban and rural pedestrian crash areas, and the alcohol-impaired pedestrian.

Urban: The Snyder and Knoblauch (1971) study was focused totally on urban crashes. Their sampling was conducted in 13 of the Nation's largest cities such as New York, Chicago and Baltimore. In addition to the published report, Snyder and Knoblauch (1971) provided NHTSA with a computer database and thorough code book. Each subsequent project done in the urban pedestrian area therefore had access not only to the Snyder and Knoblauch (1971) report but also to the collected data for guidance and information on the crash types being addressed.

Rural/Suburban: Even though Snyder and Knoblauch (1971) focused only on urban pedestrian crashes, it was known from State crash data files that pedestrian crashes were also occurring in rural and suburban areas. Therefore, the crash-typing methodology was extended by Knoblauch (1977) to cover events in rural and suburban areas. Data on a stratified random sample of over 1,500 rural and suburban crashes from six States were collected during interviews and on-site observations. The major urban types were found to exist in the rural/suburban area also along with several newly-identified types that were specific to the rural/suburban areas. In all, 23 pedestrian crash types were identified, with the 6 most frequently encountered types accounting for over $60 \%$ of the sample. 
Alcohol: The final problem identification project of the decade focused on alcohol use by the pedestrian - a critical predisposing factor noted by Snyder and Knoblauch (1971). Although suspected of having a major influence on pedestrian crashes, alcohol use by pedestrians had not been examined in great detail. A review of the literature by Zylman, Blomberg, and Preusser (1973) confirmed this lack of knowledge. Finally, a landmark study by Blomberg, Preusser, Hale, and Ulmer (1979) determined that alcohol was heavily involved and a major causal factor in adult (age 14 and older) pedestrian fatalities and injuries. In addition to finding a high relative crash risk due to the ingestion of alcohol, this study found that the blood alcohol concentrations (BACs) of drinking pedestrians involved in crashes were extremely high; approximately $50 \%$ of those who had been drinking had BACs of .20 grams per deciliter or higher. It is also of interest that the pedestrian relative risk curve developed by this study was essentially the same shape as the risk curve for drivers, but the increases in risk occurred at higher BAC levels for pedestrians. The assumption here is that walking is a simpler task than driving and, therefore, more resistant to the deleterious effects of alcohol.

\section{Countermeasure Development and Evaluation}

Based on the function/event sequence model developed by Snyder and Knoblauch (1971), a crash countermeasure can work in one or more of the following ways:

- By eliminating the human failure/error (e.g., by teaching a child how to search before crossing);

- By adding a new behavioral requirement for the other person in the sequence to compensate for the errors of the person who fails, thereby avoiding simultaneous failures and a crash (e.g., requiring all drivers to stop before passing an ice cream truck so that a child darting out will be seen and not struck);

- By changing the physical environment to make the error less likely to occur (e.g., in the commercial bus stop crash type, the bus stop is moved from the near side of the intersection to the far side, thus reducing the need for pedestrians to cross in front of the bus where they are blocked from an oncoming driver's view).

NHTSA and the FHWA share responsibilities for pedestrian and bicyclist safety. NHTSA's program responsibilities led it to focus on three kinds of countermeasures: training programs in which there is a direct transfer of information to the intended audience, public information and education (PI\&E) such as public service TV announcements, and model traffic safety regulations (laws, ordinances and rules). The regulatory countermeasures related to NHTSA's traffic enforcement responsibility. Countermeasures affecting the street and highway environments (signals, signs, markings, etc.) were pursued by FHWA. Both agencies cooperated extensively by sharing the cost and management of both pedestrian and bicyclist projects. However, the initial projects were largely single-mode efforts, e.g., just PI\&E, because they were serving the dual purpose of exploring the viability of the modality as well as developing useful interventions.

NHTSA's countermeasure development research focused on eliminating or nullifying the effects of the identified errors leading to crashes. This was accomplished by following a threestep cycle: 
1. Conceive of a potential behavioral solution and build it into one of three countermeasure forms - training, PI\&E, model regulations.

2. Conduct a limited test of the countermeasure to see if it changed the specific crashproducing behavior(s). If the behavior change results were positive, then,

3. Conduct a more complex crash reduction test with entire cities often used as "test beds."

Although this three-step process could not be used every time with all countermeasures, it was the model of choice and succeeded in identifying, developing, and evaluating several effective countermeasures that reduced particular crash types by $20 \%$ to $77 \%$. Initial targets included those crash types with the largest incidence such as the dart-out among children. They also covered crash types that seemed to have unique and straightforward solutions such as the ice cream vendor and multiple threat types. Some countermeasures were evaluated by crash reduction results, while others were judged effective because they induced desired behavioral changes with no detrimental side effects. If a countermeasure was shown to be effective, extensive support material was produced to promote its use.

Traffic Engineering: One of the first studies done in response to the findings of the Snyder and Knoblauch (1971) work was a joint NHTSA and FHWA project to examine the effectiveness of some of the traffic engineering countermeasures proposed in that work. Berger (1975) derived nine engineering countermeasures from the Snyder and Knoblauch (1971) study and tested them in one or more of eight large cities across the United States. Since the crash types were behaviorally based, Berger was able to select relevant behaviors for observation and measurement as measures of effectiveness. Included were: preventive markings ("CAUTION" painted on pavement); median barriers (chain link fence on median); crosswalk setbacks (crosswalk moved 20 feet toward midblock); diagonal parking (30- or 45-degree diagonal parking); meter post barriers (barriers extending some 3 to 9 feet in each direction from a meter); stop line relocation (stop line moved back from crosswalk); vendor warning lights (flashing signal with silhouette of child placed on top of ice cream trucks); and bus stop relocation (bus stop moved to far side of intersection). A series of studies evaluated the behavioral effects associated with the installation of a countermeasure by pairing each experimental site with a control site in a pre-post design. The study measured 30 behaviors at each site and found that 17 of them were significantly modified by the experimental countermeasures. A second task of this contract established a pedestrian crash data collection system in six cities. By combining the police crash report form and a supplementary information form, Knoblauch (1975) was able to establish a database consisting of the pedestrian crash types. Finally, a third task of the (Berger, 1975) study reported on the results of a survey of 48 ongoing education programs being conducted in eight U.S. urban centers.

Behavior Measurement: Considering pedestrian crashes based on the developed crash typology led to a need to structure the wide range of possible pedestrian and driver behaviors to focus in on those most related to the assessment of behavioral change. Rose, Levine, and Eisner (1976) took on this methodological exercise. They examined a complete range of behaviors and measurement systems involved in assessing countermeasure effectiveness for the crash types. A categorization of behavioral items was developed which included only search and locomotion behaviors, since these are the only observable events in the crossing situation. Using five parameters of pedestrian searching behavior and four parameters of pedestrian locomotion, the 
authors judged which of these parameters were likely to be significantly impacted given a set of test situations made up of 11 selected crash types and 24 potential countermeasures. Various measurement systems were evaluated. The end result guides the user through a series of steps enabling the user to identify the critical behaviors to measure for the purpose of evaluating the impact of a particular countermeasure on a particular type of crash and to determine the most cost-effective systems to be used for measuring those behaviors.

PI\&E and Training: As part of the focus on the dart-out crash type among young children, NHTSA launched the development and evaluation of both PI\&E and training countermeasures. Using the Snyder and Knoblauch (1971) data, two independent research groups detailed the characteristics of the dart-out type. They noted that the primary failure was that children were entering the roadway midblock without stopping to search for oncoming vehicles. Often they were crossing from between parked vehicles. In many cases, their entries into the roadway were not purposeful crossings, but the result of a continuation of play activities. It was apparent that the distinction between the safe and unsafe parts of the roadway was not being made by the crash-involved children. With this information at their disposal, the two research teams came up with the same advice: Stop at the curb and look left-right-and-left again before entering the roadway. Additional advice was provided to handle the cases of parked cars (stop at the edge of the car and look left-right-left) and the case of an interrupted sequence (start all over again and look left-right-left until no cars are coming on each look). This left-right-left search sequence became fundamental to both pedestrian and bicycle safety countermeasures for NHTSA and most other safety groups.

Two ways of imparting this basic advice to elementary school populations were tested. One approach (Blomberg \& Preusser, 1975) combined classroom presentations of filmed safety messages using an animated character "Willy Whistle" (a traffic policeman's whistle) with storyboards of proposed commercially-televised public service announcements to reach children at home. The child messages proved successful in changing dart-out behavior. In the same project, adult messages were also pretested and showed a behavioral change in the desired direction.

The other project (Dueker, 1975) took a more traditional training approach. It developed and field-tested a kindergarten through grade 3 street-crossing training program for use in the elementary schools. This project embodied the same behavioral advice as the "Willy Whistle" child safety messages, but used in-class and street-side practice, feedback from teachers, and a "hands on" approach. It too was able to change dart-out-related behaviors. Both projects proceeded on to larger scale evaluations in the 1980s.

Traffic Safety Regulations - Enforcement: NHTSA has responsibility for countermeasures dealing with traffic safety regulations and enforcement efforts. In the late 1960s, Singer (1969) reviewed the relationship between pedestrian enforcement and the incidence of pedestrian crashes. He concluded that most of the efforts were almost totally aimed at the driver with very little aimed at the pedestrian-driver interaction. Singer's attempts to measure the effect of an increase in the level of enforcement on pedestrian violation behavior in crossing intersections proved inconclusive, possibly due to the high level of pedestrian compliance throughout the experiment. Much later in the decade, another study examined the effect of enforcement on motorist compliance with parking bans having a potential pedestrian 
safety benefit. DeBartolo, Preusser, and Blomberg (1978) found that increased enforcement can lead to improved motorist compliance with laws related to pedestrian safety.

Model Traffic Safety Regulations - Regulatory Countermeasures: The use of traffic regulations as a countermeasure approach was guided by the logic that some of the safety advice created in response to the pedestrian crash studies could be embodied into model State laws, local ordinances, administrative regulations and related procedures. These regulations would be directed at reducing specific types of pedestrian crashes by prescribing or proscribing safe behaviors. In the first study in this area (Blomberg, Hale, \& Kearney, 1974), nine model traffic safety regulations were developed to improve pedestrian safety, with each being targeted at one or more specific pedestrian crash types. Public and official acceptance of the model regulations was assessed. The project yielded a set of regulations available for field testing in other projects. The nine regulations covered ice cream vending, road work sites, freeway vehicle stops, bus stop location, parking near intersections and crosswalks, vehicle overtaking, on-street parking in new or redeveloped residential areas, pedestrian crash information and countermeasures, and backing signals. Two of these model regulations quickly received further attention: backing signals, and ice cream vending.

Back-Up Crashes: The backing-signal regulation called for an automatic audible backing-signal device that would be effective in alerting pedestrians while not contributing to noise pollution. No such device, however, was available in 1974. NHTSA therefore launched a study to develop and test a prototype (Brown \& Sutherland, 1976). The crash figures at that time indicated that an estimated 260 pedestrians were killed and over 5,000 injured by backing vehicles annually. Older pedestrians comprised the primary population at risk. Hence, their hearing characteristics and reaction times were taken into consideration when designing the warning signal. A key feature of the prototype device was its ability to sense the ambient noise level and produce a signal just slightly louder. Evaluation of the prototype system was conducted in typical parking sites using pedestrian subjects of opportunity. Although injuries and deaths were not measured, the results showed a tenfold improvement in the number of pedestrians warned of the presence of a backing vehicle.

Ice Cream Vending: The Model Ice Cream Truck Ordinance (MICTO) is a good example of the success of a traffic regulation as a countermeasure. The problem as identified by Snyder and Knoblauch (1971) involves a young child being struck going to or from an ice cream truck vending in the roadway. The truck itself predisposes the crash because it acts as a big visual screen that prevents a driver from seeing the child pedestrian. The child, preoccupied by the prospect of ice cream, fails to search and pops out suddenly into the path of the striking vehicle. Key among the ordinance's features was the combined use of flashing lights on the vending vehicle and the activation of a STOP swing arm when stopped for vending. The ordinance required motorists to stop (then go if safe) before passing the vending ice cream truck. A field test of the ordinance in Detroit, Michigan, demonstrated a $77 \%$ reduction in the ice cream truck type of crashes (Hale, Blomberg, \& Preusser, 1978). The study also gathered information on the effectiveness of swing arms that proved useful in recommending this technology for universal use on school buses.

Safety Messages: There was some initial concern about the potential effectiveness of safety messages (PI\&E) in general when the pedestrian research program first began. Initial research suggested that messages could be effective if the messages themselves had two critical 
characteristics. First, the messages had to address specific behaviors and not be general exhortations to be safe. Second, the behaviors being promoted could not require a major lifestyle change for the recipient such as quitting smoking or losing weight. If messages successfully embodied these two characteristics, the theory was that a simple ("low cost") change in the recipient's behavior could have a large payoff in personal safety. In the first study to test this approach, Blomberg and Preusser (1975) developed safety messages based on a reanalysis of the Snyder and Knoblauch (1971) database. These were then pre-tested to determine if a behavioral change would result when the message content was received. Seven messages were pretested to measure behavioral change, and five yielded significant positive shifts in behavior. Two of the successful messages included: "Stop at the curb and look left - right - left" and "Look at the driver, not just the car." The researchers concluded that PI\&E (safety messages) can influence pedestrian behavior and provide a viable countermeasure approach to pedestrian crashes.

Bicycle Countermeasures: When the bicycle problem types became available, NHTSA decided to adopt a slightly different countermeasure development approach. Rather than following the approach taken in the pedestrian area of working on countermeasures for single crash types and only one countermeasure modality, e.g., safety messages assessed one at a time, it was decided to address all of the bicycle problem types in a single study. This single effort would also encompass training, PI\&E, and regulatory countermeasure development. The premise was that each crash type could be fully analyzed and dealt with as appropriate by each countermeasure modality.

The countermeasure development work was a collaborative effort, bringing together the principal investigators from the bicycle crash type identification work and some of the most successful pedestrian countermeasure development efforts. This long-term effort started in the 70s and produced a three-volume report (Blomberg, Leaf, Hale, Farrell, \& Cross, 1982), one in each of the countermeasure areas (training, PI\&E, and model regulatons). In total, the project developed in prototype form: three programs suitable for use as training material; a set of $10 \mathrm{TV}$ spots in storyboard form, four radio scripts, and a camera-ready reproducible of a safety poster; and eight model State laws or municipal ordinances.

In an interim report, this same project examined bicyclists' inclination and ability to search behind them before turning left (Casey, Cross, Leaf, \& Blomberg, 1980). This was prompted by the relatively high incidence problem type in which a bicyclist initiates a left turn without searching to the rear or signaling. It was found that failure-to-search rearward was fairly common in the bicyclist population, but that the ability to do so (without affecting one's steering control and stability) was within the performance range of most bicyclists. These two findings led to the promotion of universal rearward searches in the proposed bicycle safety education and training programs.

The intended next step of the NHTSA bicyclist research program called for full development and test of the most promising of the prototype countermeasures to see if they reduced target crashes. As indicated previously, NHTSA funding priorities did not permit this step to be taken. Instead, the various reports were circulated to outside groups concerned with improving the safety of bicyclists so they could consider the ideas and advice in their own efforts. 


\section{The 1980s}

The work of the 1970s flowed into the 1980s with a focus on more specialized problem identification projects as well as on most of the other research categories pursued in the previous decade. Projects ran both concurrently and sequentially, with 24 studies being done during the period.

\section{Problem Identification}

Right-Turn-On-Red (RTOR): The energy shortage led to an almost universal adoption of the Western or permissive RTOR law that permits motorists to turn right on a red signal after stopping unless prohibited by a sign. NHTSA and FHWA were asked to assess the effect of RTOR on pedestrian and bicyclist crashes. The resulting study (Preusser, Leaf, DeBartolo, \& Blomberg, 1981) collected data from New York, Ohio, and Wisconsin, and the cities of Los Angeles and New Orleans. Pedestrian and bicycle crashes involving a motorist making a right turn at a signalized intersection increased significantly at all study sites after the adoption of western RTOR. The frequency of RTOR pedestrian and bicyclist crashes was estimated and the characteristics of these types of crashes determined. The study identified some problems and prompted new research by FHWA into countermeasure solutions.

Child Pedestrian Supervision: Prior NHTSA research indicated that the lack of adult supervision was a major predisposing factor for many crashes involving pre-school and elementary school-age children. This situation was examined in-depth by Thackray and Dueker (1983) who identified and evaluated the crash risk associated with play activities performed in and near the street in San Francisco. Supervision strategy countermeasures were identified and implementation plans specified.

Conspicuity: Something that is conspicuous is obvious to the eye or mind and has the capability of being easy to see. Conspicuity is a concern that affects pedestrian and bicyclist crashes of many kinds both in the daytime and at night. Uncovered as a predisposing factor in the Snyder and Knoblauch study (1971) and confirmed by Cross and Fisher (1977), work on it began within the program with a review of the literature by Hale and Zeidler (1984). This was followed by a study (Blomberg, Hale, \& Preusser, 1984) that conducted tests to assess the effectiveness of various material and strategies for enhancing the nighttime visibility of these road users. Data was collected from subjects driving along a course who were requested to indicate when they detected an object and also when they recognized the object as a pedestrian or bicyclist. Classic advice such as "wear white at night" proved to be ineffective, and was replaced with other recommendations. Outlining the human form with retroreflective material so that the shape of a person was conveyed aided identification. Also, if someone must bicycle at night, an active light source supplemented by at least the standard retroreflectors should be used. A high-intensity bicycle lighting system would be useful for those who regularly ride at night. Flashing lights appeared to be a reasonable choice for both pedestrians and bicyclists.

\section{Countermeasure Development}

Training of Rural/Suburban Children: A major training countermeasure project called PedSafe was addressed at the pedestrian problems identified by Knoblauch (1977) in the rural/suburban area. A series of reports (Chiplock \& Dueker, 1981; Bittner, Chiplock, \& Dueker, 1981; and Chiplock, Dueker, \& Bittner, 1981) covered the development and test of a 
pedestrian safety training program to combat pedestrian crashes victimizing suburban and rural school children. The training covered kindergarten through twelfth-grade children and a multitude of crash types. User guides, teacher guides, and student booklets were prepared along with scripts for all of the audiovisuals used in the program. Of particular interest was the work done on a school bus safety program; it was later expanded upon (see Cleven \& Blomberg, 1994) to form a program distributed nationally by the National Safety Council under the name WalkRide - Walk: Getting to School Safely.

Training of Preschoolers: The young preschool child is over-represented in pedestrian crashes, and traffic crashes are a leading cause of death for the child under 6. A study was undertaken by NHTSA to develop a traffic safety program that could reduce the occurrence of pedestrian crashes for preschoolers (under 4 years old). Efforts began with a literature review (Phinney, Colker, \& Cosgrove, 1985), which was used to facilitate the development of a countermeasure program. After an analysis of thousands of preschooler crash reports, a set of safety education material was developed by Applied Management Sciences, Inc., in 1985, for preschool educators and parents. The Headstart program of the Health and Human Services Agency was the first to use this product. The National Association for the Education of Young Children, under a grant from NHTSA, revised the curriculum, and developed a companion program entitled Children Riding on Sidewalks Safely (CROSS). The Walking in Traffic Safely (WITS) program was made available through the National Association for the Education of Young Children. Although this product was used by different organizations, it was never formally tested.

Model Regulations - Rural/Suburban: Four prototype model regulations and four media packages were developed (Hale, Blomberg, \& Kearney,1980) dealing with rural/suburban pedestrian crash problems identified by Knoblauch (1977) and with freeway pedestrian crashes researched by Knoblauch, Moore, and Schmitz (1976).

Model Regulations - Dismounted Motorist, Road Workers: Two of the model regulations that were developed (Hale et al., 1980) received further attention (Ulmer, Leaf, \& Blomberg,1982). One regulation was concerned with the disabled-vehicle situation where a driver has to exit the vehicle to make a repair, flag down traffic or walk along the roadway to obtain assistance. This model regulation calls on motorists to position themselves and their vehicles as safely as possible and to employ conspicuity-enhancing devices and materials. When studied experimentally in the field, it was shown that deploying fusees or warning triangles in conjunction with four-way flashers significantly reduced the speed and shifted the placement of vehicles passing a simulated disabled vehicle during daytime and nighttime conditions. On the other hand, wearing fluorescent and retro- reflective materials did not appear to influence the course or speed of passing motorists. Work on a model regulation for road workers was halted when a detailed analysis of the crash reports indicated there was a variety of causes and that these crashes were made up of several sub-types. It was concluded that only a minority of road worker crashes would be affected by any single model regulation.

Model Regulations - School Bus Pedestrians: A prototype model regulation for school bus pedestrians was also examined (Ulmer et al., 1982). School bus driver experiences and school bus passing violations were analyzed to assess the potential effectiveness of this regulation. Safety features such as special school bus equipment (amber and red signal lights, STOP swing-arms and pedestrian crossing mirrors) and driver procedures to prevent children 
from being struck while crossing to or from a school bus by other vehicles or by the school bus itself were assessed. A revised model school bus regulation, incorporating these results, along with concepts for PI\&E to support compliance, was developed.

PI\&E - All Age Groups: A project by Preusser, Blomberg, Edwards, Farrell, \& Preusser (1985) focused on pedestrian crash types and situations not previously addressed through public education. These included "riding toys," "backing," "pedestrian not in road," "visual screens," "intersection dash," "darts and dashes," "school bus," "child supervision," "elderly," "mail box," and "disabled vehicle." Each type was analyzed to determine specific behavioral advice that could be adopted by pedestrians, parents or drivers. Prototype TV and radio scripts, pamphlets and posters were developed to carry this advice to identified target groups. These prototype media forms underwent focus group reaction testing. Three TV spots and a 15-minute in-class film were produced in finished form.

\section{Countermeasure Evaluation}

PedSafe Training Program: The PedSafe program, a pedestrian safety training program for rural and suburban children, received a feasibility test of its elementary, on-bus, and junior/senior high school components (Dueker \& Chiplock, 1981). The elementary and on-bus programs addressed four pedestrian situations - midblock crossing, intersection crossing, walking along the roadway, and pedestrian movement near the school bus. Statistically significant reductions in unsafe pedestrian behavior brought about by the elementary and on-bus programs were found, using a pre/post with comparison design. A similar design was used to evaluate pedestrian safety knowledge gains for the junior/senior high school program. Statistically significant gains resulted from all five units within this program.

Anti-Dart-Out Training: The in-class training program developed by Dueker (1975) to combat dart-out crashes among elementary school children underwent an evaluation when Dueker (1981) tested two alternative versions. One version, the film program, was evaluated in the Toledo Public Schools, while the second version, the film/simulator program, was evaluated in the New Orleans Public Schools. The film program proved to be superior, exhibiting a $20 \%$ reduction in crashes. The program material and staff training material are found, respectively, in Volumes 2 and 3 of Dueker and Berger (1981).

Model Regulations - Parking: Three model traffic safety regulations developed by Blomberg et al. (1974) were examined. Two of the model regulations sought to prevent crashes characterized by pedestrians darting into the street and being struck by motorists who are screened by parked vehicles and have inadequate time to react. The regulations prohibited onstreet parking in certain circumstances (midblock and near intersections) in order to remove the parked vehicles which screen pedestrians and motorists from seeing each other. The study made use of residential areas of Manhattan, New York, which had alternate-side parking. Analyses of 835 pedestrian crashes over a four-year period indicated no changes when the alternate-side parking regulation was in effect. The authors (Leaf \& Blomberg, 1981) felt that little could be concluded because of discrepancies between the New York study situation and the model regulations. In a related activity, support material was prepared for a third model regulation, one requiring motorists to stop prior to proceeding past another vehicle stopped before a crosswalk (in an attempt to prevent the "multiple threat" crash situation). 
PI\&E-Children: The safety messages aimed at preventing dart-out behavior among children (Blomberg \& Preusser, 1975) had reached the stage where they could be tested for their crash-reduction ability. The child anti-dart-out messages, which included a 6- to 7-minute classroom film, three 30 -second and three 60 -second TV spots, and a poster, all employed an original animated character named Willy Whistle as the spokesperson. The six TV spots covered each of the behavioral messages contained in the classroom film. A field test (Blomberg, Preusser, Hale, \& Leaf, 1983) was undertaken to determine if the messages were effective countermeasures for dart-out crashes among young children. The material was distributed to television stations and schools in Los Angeles, Columbus, Oio, and Milwaukee, Wisconsin. The messages received good exposure, and created increases in safe-street crossing knowledge and improvements in actual crossing behaviors in all three cities. The most important change was a statistically significant reduction of over $30 \%$ in dart-out crashes among children in all of the cities. In a separate study, an evaluation of a film entitled And Keep on Looking designed to reduce pedestrian crashes among 9- to 12-year-olds found an increase in safe street-crossing knowledge, some improvement in safe street-crossing behavior, and a crash reduction of more than 20\% (Preusser \& Lund, 1988).

PI\&E - Adults: The development and test of adult safety messages were also developed and tested concurrent with the child messages (Blomberg \& Preusser, 1975; Blomberg et al., 1983). Two adult crash types - the vehicle turn/merge (VTM) situation and the multiple threat (MT) situation - were addressed with two 60-second and two 30-second TV spots, and with 60and 30-second radio spots. The VTM message for drivers reminded them to take a last look for pedestrians before making turns at intersections. Pedestrians were cautioned to look at the driver not just the car when crossing near cars making turns. These VTM messages were produced in both English and Spanish and tested in Los Angeles and San Diego. For the MT messages, drivers were told to look for cars stopped in the traveled lanes, slow down, and ask themselves why the car was stopped (car could be hiding a pedestrian). The message for pedestrians was to stop at the edge of any car that stops to allow a crossing, and to look around it for any cars coming in the next lane. The MT messages were in English only, and were tested in Los Angeles. The results were not overly favorable, but the overall pattern of knowledge, behavior, and crash data do suggest that message effectiveness increases with increased exposure. The Spanish language VTM messages proved most effective, yielding a statistically significant crash reduction of $18 \%$. Heavy competition in the adult public service announcement market provided much less exposure for the adult safety messages than for the child messages discussed earlier. The overall conclusion was that the adult messages were still viable countermeasures, particularly when sufficient exposure could be generated.

Program Development: There were a number of studies done in the 80 s that are more properly classed as "program development" projects. They focused on objectives such as assessing the public's acceptability of proposed countermeasures (Vayda \& Crespi, 1981), conducting a state-of-the-art review of the pedestrian crash area (Fell \& Toth, 1981) and reporting to the Congress on the effectiveness and efficiencies in the pedestrian safety area (Ehrlich, Farina, Pavlinski, \& Tarrants, 1982).

Program Assessment Kit (PAK): One of the more encompassing projects of the 1980s was the development of a package of material - the Program Assessment Kit (PAK) - to be used in assessing the safety relevance of pedestrian and bicyclist safety education programs (Blatt \& Dueker, 1983). The rationale for developing the PAK was to provide State and local safety 
practitioners with a paper-and-pencil survey for assessing the safety relevance of pedestrian and bicyclist safety education programs. The safety relevance of a given program is the extent to which its content reflects the findings of NHTSA research in crash causation and countermeasure development. The PAK can also be used as an aid to the improvement of existing programs and the development of new ones.

\section{The 1990s}

Problem identification projects were still being undertaken in the 1990s, again on more circumscribed topics than in the 1970s, e.g., the distribution of the alcohol pedestrian problem among the diverse cultural groups in the United States; and determining the relationship between vehicle speed and pedestrian injury. A major integrated pedestrian countermeasures project was also started in Miami-Dade County, Florida.

\section{Problem Identification}

Crash Types: Since over 20 years had elapsed since crash types were first identified, it was decided to examine the prevailing types of the 1990s to see if there had been any significant changes (Hunter, Stutts, Pein, \& Cox, 1995). The pedestrian and bicycle crash types originally identified were still very much in evidence, although the relative percentages of some types had shifted slightly. No major new types were identified. This gave even more impetus to the use of the previously developed countermeasures and to the development of the Pedestrian and Bicycle Crash Analysis Tool (PBCAT) to assist crash typing.

Alcohol Diversity: Half of adult pedestrian fatalities involve people who had been drinking. Leaf and Preusser (1997) examined the extent of involvement of racial/ethnic groups in this problem. Racial data were obtained for all 1987-89 data from the Fatality Analysis Reporting System (FARS) and for 1 to 12 years of FARS data for seven States or State subsets. Analyses showed three specific groups with pedestrian-alcohol fatality risks as high as or higher than the population as a whole: Black adults 25 and older, Hispanic adult males, and Native American adults. Focus groups were conducted with all three groups on the use and abuse of alcohol within their cultures, and reactions were obtained to 28 specific countermeasure themes such as police enforcement, public education, engineering, laws, alcohol vendor and server practices, and enhanced conspicuity.

Speed and Pedestrian Injury: The relationship between vehicle travel speeds and resulting pedestrian injury was also examined (Leaf \& Preusser, 1999). The study was accomplished through a review of the literature and an analysis of existing data sets. Results indicated that higher vehicle speeds were strongly associated with serious pedestrian injuries and a possible increase in the occurrence of pedestrian crashes. It was estimated that $5 \%$ of pedestrians would die when struck by a vehicle traveling at $20 \mathrm{mph}$ or less. This compares with fatality rates of $40 \%, 80 \%$ and nearly $100 \%$ for striking speeds of 30,40 and $50 \mathrm{mph}$, respectively. The authors reported that reductions in vehicle travel speeds can be achieved through lowered speed limits, police enforcement of speed limits and associated public information. More long-lasting speed reductions can be achieved through traffic calming approaches. There was little information available from the literature on the speed reduction effectiveness of combining education and enforcement in traffic calmed and non-traffic-calmed environments. 


\section{Countermeasure Development}

Older Pedestrians PI\&E: Older adults are particularly vulnerable as pedestrians. Blomberg, Cleven, and Edwards (1993) focused on the pedestrian risks facing older adults (65+) and ways of safeguarding this increasing segment of the population. Prior research was reviewed and recent crash data sets were analyzed to identify the types of crashes involving older pedestrians. Pedestrian safety messages were then developed for each of four defined crash situations in which the elderly are involved — vehicle turn/merge, turning vehicles, backing vehicles and "other intersection" crash types not involving turning vehicles. In addition, it was confirmed that elderly crashes increase markedly in the winter months when the sun angle is lowest. This increase appears to be a problem of conspicuity. The risks and behavioral advice were documented in a white paper titled Walking Through the Years. Additional supporting material was developed to communicate this information to groups of older adults. Finally, organizations capable of reaching large numbers of older adults were identified and contacted for their cooperation in disseminating the safety information.

\section{Countermeasure Evaluation}

School Bus Safety Training: The actual school bus trip to school is among the safest forms of transportation, but there are substantial pedestrian risks associated with the total trip as the child walks to and from the bus stop, waits for the bus in a traffic environment, gets on and off the bus at home and at school, and sometimes crosses the street to and from the bus. It was determined that the school bus work done by Dueker and Chiplock (1981) in the rural/suburban area needed to be updated and expanded to include the urban sector. This task was successfully undertaken by Cleven and Blomberg (1994), who reviewed existing material, crash data, and State laws and regulations on school bus pedestrian safety. They developed a program incorporating 113 behaviors covering major categories, such as getting ready for school, walking to/from the bus stop, waiting at the bus stop, crossing to the bus, boarding the bus, riding the bus, exiting/crossing from the bus, and evacuating the bus. Included in the program was material for teachers, parents, and bus drivers. Separate teacher's guides were developed for each of the seven grade levels (kindergarten through grade 6). Three videos were developed specifically for the program (for students, parents, and bus drivers). The program also made use of two previously-produced NHTSA videos that have demonstrated their crash reduction ability in earlier studies. The program was tested in a New York State school district and was favorably received. The research product underwent a product-enhancement process that created an attractive commercial product totally contained within a miniature school bus. The program, called Walk-Ride-Walk: Getting to School Safely, was made available through the National Safety Council, which sold more than 4,000 copies to schools nationwide at a nominal cost of $\$ 55$ each.

Regulations - RTOR Revisited: At the request of the Congress, NHTSA conducted a study of the safety impact of permitting right- (RTOR) and left-turns-on-red-lights (LTOR). The earlier study of RTOR by Preusser et al. (1981) had indicated that the law put pedestrians and bicyclists at increased crash risk. The new study (Compton \& Milton, 1994) examined both RTOR and LTOR. At the time of the study, 43 jurisdictions provided for LTOR and 9 did not. LTOR is actually permitted only at intersections of a one-way street with another one-way street. Thus, there are relatively few intersections where a LTOR is permissible. The authors found so little data among FARS and State data system records concerning LTOR crashes that no analysis of its impact was possible. From their analyses of RTOR data, the authors concluded there is a 
relatively small number of deaths and injuries each year caused by RTOR crashes. When a RTOR crash did occur, a pedestrian or bicyclist was frequently involved and nearly always sustained injury. From the point of view of total crashes (vehicle-vehicle and vehiclepedestrian/bicyclist), Compton and Milton (1994) concluded that because the number of crashes due to RTOR is small, the impact on traffic safety, therefore, has also been small.

PI\&E/Engineering - Older Pedestrian Zones: This joint NHTSA/FHWA study developed procedures for creating pedestrian safety zones within communities around areas of high crash frequency for older pedestrians (Blomberg and Cleven, 1998). The zones were saturated with an appropriate mix of engineering and educational countermeasures. Zoning allowed for a more efficient use of countermeasure resources, e.g., $55 \%$ of the crash problem was treated by focusing on less than $5 \%$ of the city's land area. The study was conducted in two cities: Phoenix and Chicago, but a complete crash evaluation was conducted only in Phoenix. Data from Phoenix showed a statistically significant $46 \%$ reduction in in-zone crashes to $65+$ pedestrians over a period in which the city's population and overall pedestrian crashes increased. The study also resulted in a Zone Guide for Pedestrian Safety designed to assist program implementers in other cities in defining zones and applying the zone process to their pedestrian safety problems.

\section{Program Material}

A number of 1990 s projects related not to the development or test of specific countermeasures, but to issues related to the broader program, including the development of program material. These are described below.

Community Assistance: Walk Alert is a national pedestrian safety program developed for FHWA with material and financial input from NHTSA, and safety material contributions from over 100 service organizations and many community groups. The American Automobile Association and the National Safety Council were co-developers of the program along with the federal government. It is a comprehensive program addressing pedestrian safety at the community level by using the three "E's" of Education, Engineering, and Enforcement. Walk Alert was specifically designed for safety volunteers, concerned citizens, grass roots service organizations, and city and county governments.

Program Review: During the period from 1983 to 1989, little research was done within NHTSA in the pedestrian and bicyclist safety behavioral research area due to policy decisions at the agency level to devote resources to increasing use of safety belts and child restraints and to decreasing alcohol-impaired driving. In the early 1990s, a project was initiated to "fill in the gap of these NHTSA-dormant years" by reviewing key programs and countermeasure developments occurring outside NHTSA. The resulting report by Stutts, Hunter, Tracy, and Wilkinson (1992) provided a review of key countermeasure developments and program activities impacting on pedestrian and bicyclist safety over the past decade. It pulled together into one document information from a wide array of sources, including Federal, State and local governments, nongovernment agencies and organizations and, in many cases, "word of mouth" communications. By identifying what had transpired, the report also identified a rich network of organizations and individuals eager to be a part of a larger pedestrian and bicyclist safety agenda. A key recommendation was that NHTSA/FHWA work in partnership with other non-government organizations to develop "an infrastructure of implementers" at the State and local levels to whom they can convey their safety products. 
Crash Analysis Tool (PBCAT): The FHWA, in cooperation with NHTSA, funded the Highway Safety Research Center to develop a Pedestrian and Bicyclist Crash Analysis Tool (PBCAT). PBCAT is a software product aimed at assisting State and local pedestrian and bicycle coordinators, planners, and engineers. It does so through the development and analysis of a database containing details associated with crashes between motor vehicles and pedestrians or bicyclists. A major feature of PBCAT is its ability to allow the user to quickly determine the pedestrian or bicyclist crash type through a series of on-screen questions about the crash. Crash typing provides useful information on the sequence of events and precipitating actions leading to the crash, thus complementing the demographic information usually available. PBCAT is adaptable and easy to use. It enables practitioners to generate valuable information for promoting bicycle and pedestrian safety and designing safer facilities where bicyclists, pedestrians and motor vehicles interact. PBCAT was revised in June 2006 to add the following improvements: enhanced navigation in a more familiar user interface, more user options and greater customization, greatly enhanced countermeasure information, better reporting capabilities, reduced number of crash types, more location detail, and "advertised" product support.

Improving Awareness: A public unaware of a problem is unlikely to be receptive to available solutions. This is the nature of the situation facing advocates for pedestrian safety. A joint NHTSA/FHWA project was therefore initiated in 1992 to increase awareness of pedestrian safety problems on a nationwide level in order to establish a base of support for current and future safety programs aimed at reducing the problems. It was realized early on that neither agency had the resources to mount large public information campaigns that might address the problem. Networking with a host of other organizations was therefore a necessary step. The contractor, Highway Safety Research Center, in association with Dunlap and Associates, Inc., the Pedestrian Federation of America, and the Center for Applied Research, responded by creating a new national organization - the Partnership for a Walkable America. The partnership was a national coalition working to improve conditions for walking in America and to increase the number of Americans who walk regularly. The members were national government agencies and nonprofit organizations concerned about three main areas: health, safety and the environment. Members of the steering committee of the alliance were the Pedestrian and Bicycle Information Center, NHTSA, FHWA, the Centers for Disease Control and Prevention, the Institute of Transportation Engineers, and the Robert Wood Johnson Foundation. The Partnership sponsored the International Walk to School Day Program, provided walkability and bikeability checklists and presented annual pedestrian project awards. The activities of the Partnership for a Walkable America have now been taken over by the Pedestrian and Bicycle Information Center - a clearinghouse sponsored by FHWA with support from NHTSA.

Bicycle Safety Resource Guide: In 1994, the opportunity arose to revisit the findings and recommendations of the bicycle countermeasures development study by Blomberg et al. (1982). A joint NHTSA/FHWA project was conducted by Dunlap and Associates, Inc., under contract with the Highway Safety Research Center to analyze the then current bicycle safety problem and develop countermeasures to impact that problem. Contacts with numerous bicycle safety professionals revealed that many countermeasures had been developed and much work had been done on bicycle/motor vehicle crash types, but little had been done to simplify and put into operation the relationship between problems and remedial actions. It was decided that people responsible for bicycle safety at the State and local levels could benefit from the availability of a 
comprehensive resource guide that identified countermeasures that could be carried out by the different implementers, e.g., police, traffic engineers, who are often part of local safety efforts. A guide was therefore developed that identified 40 different bicycle problem areas and the countermeasures that could be used by 15 different implementer groups to help reduce each problem area. Where no countermeasures were located, ideas for new countermeasure development were included in the guide. Therefore, the bicycle safety professional could enter the guide with a problem area and identify existing or proposed countermeasures that could be used to help combat that problem by each of the 15 implementer groups. In all, 226 existing and 75 proposed countermeasures were described. The bicycle guide (also sponsored by FHWA) was produced and disseminated in CD-ROM format (Dunlap \& Associates, 1998).

\section{The 2000s}

Starting in the late 1990s and continuing into the 2000s, NHTSA sponsored a variety of bicycle and pedestrian studies, some of which are still ongoing. Among others, these have included guides on laws affecting pedestrian and bicycle safety, a pilot field study of speed and pedestrian safety, and updates of prior successful NHTSA-sponsored research.

Alcohol and Pedestrians: Alcohol has been found to be involved in 35\% to $45 \%$ of all adult pedestrian crashes. It is the single largest pedestrian crash problem. A study in Baltimore) developed and tested a set of countermeasures designed to reduce alcohol-involved pedestrian crashes (Blomberg \& Cleven, 2000). This study, known as Walk Smart Baltimore, thoroughly analyzed alcohol-involved pedestrian crashes in the city, and zones having high crash frequency were identified. With the cooperation of multiple city departments, a program was put in place to raise the awareness of relevant players (drivers, the alcohol-involved pedestrian, alcohol servers, the general public) about the problem, make engineering corrections at crash sites, train the enforcement community on what to look for and what to do, and improve the conspicuity of the drinkers at night. Since "had been drinking" was not routinely checked on police crash reports in the city, a surrogate measure based on victim age, time of day and day of week was developed to estimate the occurrence of a pedestrian alcohol crash. Time series analyses conducted citywide did not show a statistically significant reduction in alcohol related pedestrian crashes. However, substantial reductions for a surrogate group in total crashes, zone crashes, and crashes on roads on which special signs were erected suggested the study made some positive inroads into reducing the pedestrian alcohol problem in Baltimore. The surrogate group consisted of victims whose personal and crash variables were highly associated with victims that had been drinking.

Laws Related to Pedestrian and Bicycle Safety: A Resource Guide on Laws Related to Pedestrian and Bicycle Safety was developed (Dunlap \& Associates, 2000). The guide presents a selection of "key" vehicle and traffic law provisions drawn from three sources: the Uniform Vehicle Code prepared by the National Committee on Uniform Traffic Laws and Ordinances; other State laws and local ordinances that are perceived to have a positive or negative effect on pedestrian or bicycle safety; and model laws prepared by this project to meet specific safety needs. The safety relevance of each key provision was assessed in terms of its likely effects on the causes of bicyclist or pedestrian crashes with motor vehicles and the prevention or reduction of bicyclist or pedestrian injuries. Each key provision was cross-referenced to a description of how that concept is implemented in the 50 States. Produced in CD-ROM format and also 
available as a download from the NHTSA Web site, the guide provides an easily accessed database of current and proposed laws that may affect pedestrian and bicycle safety.

Reducing Speeds in Neighborhoods: Some communities have used traffic calming on selected streets in a neighborhood but left others untouched because of objections voiced by emergency services. This has created streets within a defined calmed neighborhood where motorists continue to exceed prudent speeds - or at least exceed the speeds on adjacent streets. The objective of this study (Blomberg \& Cleven, 2006) was therefore to attempt to achieve on these untreated streets the level of speed reduction achieved on the adjacent streets that have received traffic calming treatments. Three neighborhoods each in two cities, Phoenix and Peoria, Arizona, were selected for a program known as Heed the Speed. Countermeasures included PI\&E, enforcement, and various roadway treatments. The program was evaluated by a pre/post mailed survey, by police data forms completed at each stop and by multiple waves of speed measurements using on-road traffic counters. The survey showed a strong increase in knowledge of the program and the need to moderate speeds. Respondents also expressed a strong belief that speeds in their neighborhood had decreased since the Heed the Speed program was implemented. The police records showed that most violators were neighborhood residents. Speed measurements showed significant reductions in all six neighborhoods and on all test roads within the neighborhoods except one low volume street with pre-existing speed humps installed. PI\&E and enforcement were associated with significant decreases in speed both on calmed and streets that were not calmed.

Bicycle and Pedestrian Safety Resource Guide: NHTSA previously sponsored the development of resource guides of both bicycle and pedestrian safety resource material. The bicycle guide (also sponsored by FHWA) in CD-ROM format was well received and widely distributed. The pedestrian guide was prepared in CD-ROM format in 2002 but was not formally produced or distributed. With the ever-increasing production of safety material for both pedestrians and bicyclists, both CD-ROMs became out-of-date. The updated guide was released recently in both CD-ROM form and as a Web site download (Dunlap \& Associates, 2006).

\section{Products in 2007}

Comprehensive Programs: The Miami-Dade Project: Pedestrian crashes are a serious problem in U.S. cities. The risks in the Miami-Dade area were particularly challenging, not only because of the number of crashes - over 1,700 per year -- but also because the ethnic and age diversity in the region made intervention more problematic. The problem was recognized by local officials, and NHTSA saw this setting as an opportunity to apply and test an aggressive, data-intensive approach to pedestrian crash reduction based on extensive NHTSA and FHWA research projects. The effort was led by the University of North Carolina's Highway Safety Research Center and was supported by the Florida Department of Transportation and the MiamiDade County Metropolitan Planning Organization. Data was used initially to identify problems and their variations across the study area, particularly the differences in patterns across ethnic and age groups. On the basis of this more-detailed problem definition effort, a repertoire of interventions was defined, matched to crash types, locations, and pedestrian-driver demographics. The interventions include educational programs (e.g., brochures, public service announcements, classroom training); enforcement (especially targeted at nighttime alcoholrelated crashes); and infrastructure enhancements (safety medians, signals, cross walks). 
Preliminary findings suggest pedestrian crash reduction occurred in the targeted safety zones where countermeasures were targeted.

Development of an Automated System for Reducing Illegal Passing of School Buses. Research has shown that approximately $60 \%$ of fatalities that occur in school bus loading and unloading zones were the result of motorists who failed to stop when the vehicle's STOP swingarm was extended and the red warning lights were flashing. To address this problem, NHTSA sponsored research aimed at developing an automated system for detecting and recording the license plates and drivers of vehicles that illegally pass stopped school buses (Hanowski et al., awaiting release). The study was conducted in two phases: initial system research and system refinement. The initial development effort was co-conducted by the Virginia Tech Transportation Institute (VTTI) and WESTAT. Refinement of the system was accomplished by VTTI. The study effort involved a feasibility analysis, development and test of an initial prototype system and refinement of that system. Recommendations were made for future study and testing. 


\section{DISCUSSION}

The NHTSA pedestrian and bicycle research program is a coherent effort that has existed for almost 40 years. During that period, the outcomes of the various research efforts have provided feedback for program improvement as well as lessons learned in several key topical areas. These lessons and the implication of the program's findings for future research are discussed in this section.

\section{Problem Identification}

The NHTSA pedestrian and bicycle safety research program adopted several novel approaches early in its existence. These included identifying behaviorally-based crash types as a foundation for countermeasure development and initially focusing on individual countermeasure modalities to determine their utility as interventions against pedestrian and bicycle crashes with motor-vehicles.

Both of these approaches have proved to be of significant value to the genesis and perpetuation of an effective and well-received program. As discussed earlier, the choice of a behavioral underpinning for the taxonomy of crashes that developed into the various crash types described in Appendix B was likely crucial. The success of multiple countermeasure programs using the behaviorally-defined pedestrian and bicycle crash types confirms that motorists and nonmotorists alike have been able to relate well to countermeasures based on the errors they are actually making.

The focus on individual countermeasure modalities also proved to be a good approach both to facilitate evaluation and for understanding how the modalities work. In particular, the NHTSA-sponsored research of the 1970s established that each of the three basic countermeasure types - engineering, education (PI\&E and training), and enforcement - could be effective when directed to the identified crash types. More recent NHTSA and FHWA activities have combined modalities seeking interventions that are highly acceptable and effective. The current thinking that the most effective and efficient programs should encompass all countermeasure types (engineering, enforcement and education) plus encouragement and evaluation ("5 E's") is certainly supported by the experience of the NHTSA program over the years.

The original crash type identification studies identified alcohol use by the pedestrian or bicyclist as a significant predisposing factor. The relative risk study by Blomberg et al. (1979) for pedestrians clearly confirmed the causal role of alcohol.

Finally, the robustness of the crash types has been well established. Even though originally identified in the 1970s, all of the available evidence suggests that there have been no major additions or deletions from the lists (see Hunter et al., 1995). The evolution of types described in Appendix B represents a refinement and simplification of the typologies rather than the emergence or extinction of any underlying phenomena. This is not surprising given that the basic function/event sequence model developed by Snyder and Knoblauch (1971) is behaviorally based. The basic human functions of search, detection, evaluation, decision and action are fundamental and not subject to major changes. 


\section{Countermeasure Development}

The original studies by Snyder and Knoblauch (1971) and Cross and Fisher (1977) indicated that pedestrian and bicycle motor-vehicle-related crashes can be disaggregated into many crash and problem types. The sheer number of crash and problem types means that countermeasures must be tailored to a multitude of specific causal factors. Alcohol countermeasures (e.g., advice such as You drink \& drive. You lose) and occupant protection countermeasures (advice such as Click It or Ticket) are directed solely at vehicle operators and occupants. Pedestrian and bicycle countermeasures can effectively be directed at both drivers and pedestrians or bicyclists because, according to the function/event sequence model, either is capable of preventing a crash. Thus, over the years the pedestrian and bicycle research program has provided useful advice for:

- Young pedestrian road users;

- Older child pedestrians;

- Older adults;

- Spanish speaking young adults;

- Information on effective conspicuity enhancing materials and devices;

- Vehicle operators including drivers who exit their vehicles and need to walk along the roadway; and

- Pedestrians and bicyclists who drink.

Addressing this broad set of audiences gave the program a breadth that proved useful in dealing with the various crash types.

\section{Evaluation}

Evaluations have been conducted over the years to assess individual countermeasure effectiveness. These indicated that progress has been made with young and older pedestrian road users and, to a lesser extent, with alcohol impaired pedestrians. Less is known about the effects of combining multiple countermeasures into an ongoing program aimed at reducing crashes in a particular jurisdiction. Information soon to be available from a comprehensive program in Miami-Dade County may prove beneficial.

Methodologically, the evaluations within the pedestrian and bicycle research program were somewhat limited by the absence of readily collectable intermediate measures of program effectiveness. Many motor vehicle offenses such as speeding and red-light running are relatively easily measured at the behavioral level. Pedestrian and bicyclist behaviors and many of the motorist behaviors critical to crash avoidance (e.g., search) are difficult to measure in a valid and reliable manner. This is an inherent characteristic of pedestrian and bicyclist activities that has been dealt with primarily by relying on crash-based evaluations.

\section{Remaining Issues}

Although the pedestrian and bicycle research program has accomplished much and created an excellent model, issues remain for future examinations. Addressing these issues with future research efforts should provide even more understanding of the pedestrian and bicycle 
crash problems and help identify additional countermeasure approaches and individual interventions that can be effective.

Identification of Valid Measures of Behavior Change: The crash generation model defined by Snyder and Knoblauch (1971) and refined by Cross and Fisher (1977) as well as virtually all of the countermeasure efforts throughout the life of the NHTSA research program have shown the importance of proper search behavior for crash prevention. Thus, in addition to crashes and crash rates, intermediate measures of effectiveness based on the visual search behavior of drivers, bicyclists and pedestrians are clearly of interest particularly as part of evaluation research. The problem is that search measures are difficult to collect in a valid manner. Much of the search performed by adults employs eye movements rather than detectable head turns. Thus, systems such as in-car cameras or relatively imprecise personal reports of behavior change should be examined.

Need for Valid Measure(s) of Exposure: The need to consider crash rates as a measure in some future research emphasizes the need for further consideration of pedestrian and bicycle exposure measures. Several NHTSA and FHWA efforts to develop pedestrian and bicycle exposure measures have not led to universally accepted metrics analogous to vehicle miles driven for drivers. Future efforts would best focus on exposure measures that are specific to the issue being studied and the ability to collect valid and reliable exposure data. For example, in the evaluation of Safe Routes to School (SRTS) programs, it is possible to collect reasonable counts of trips as a function of transportation mode. While it is not known if trip counts provide the best measure of crash risk for pedestrians and bicyclists, there should be widespread agreement that they provide one exposure measure of interest. Moreover, if pedestrian and bicycle trips increase significantly and the rates of crashes per trip do not, it may be reasonable to conclude that both the encouragement and safety components of the studied program may have had some effects.

Alcohol Research: The study by Leaf and Preusser (1997) suggests that some headway has been made in the development of countermeasures and the remediation of the pedestrianalcohol problem. Much remains to be accomplished, however, and the underlying health issues implied by the high blood alcohol concentrations characteristic of the drinking pedestrian or bicyclist victim have proved resistant to both traditional highway safety and public health countermeasures.

\section{Future Crash Reduction Efforts}

The importance of the continuing use of crash types within the NHTSA research program has been established. It is essential, however, to place crash types and the typing process in its proper place in the overall effort to reduce crashes. Early in the NHTSA and FHWA research programs, an understanding of crash types and the determination of each jurisdiction's mix of types were considered essential parts of mounting an intervention. The rationale was that the understanding would spawn effective local countermeasures and countermeasure variations, and the determination of the distribution of types at the local level would avoid wasting limited resources on problems that were not locally applicable.

A clear lesson from the NHTSA program is that, while crash typing is a useful research and evaluation tool, it may require too much effort for too little benefit to be used as a routine 
operational technique in all communities. The stability of the crash types suggests that NHTSA need not devote significant portions of limited resources of its pedestrian and bicycle research program to further verification of the types. Since the PBCAT can be used as part of ongoing research efforts and field evaluations, the resulting crash type distributions, including those crashes that cannot be classified, should be sufficient to identify if there is a need to modify existing or add new types. In particular, examining the number and proportion of crashes that cannot be classified by PBCAT within future studies should be a simple and effective way to indicate the possible emergence of new types.

A corollary to the finding that inclusion of all five "E's" strengthens an intervention is the lesson that pedestrian and bicycle safety is significantly intertwined with quality of life and health issues. Current efforts to promote SRTS programs are a prime example. These programs, although clearly inclusive of safety, have encouragement as a major theme. Reductions in obesity, improvement in cardiovascular conditioning, and lower levels of vehicular-caused pollution are all desirable and sought after outcomes of SRTS programs. Future safety research emphasis therefore needs to be recalibrated to focus on avoiding crash increases as exposure in terms of more pedestrian and bicycle trips climbs as well as on reducing crash incidence.

Examining crash rates as well as raw crash numbers for vehicle occupant fatalities must be an important part of future evaluation research.

Future efforts are needed in the area of safe street crossing advice for adults walking in highly complex environments. In addition, supervision of children needs review. Such efforts will need to go beyond traditional advice given to young children and specific advice directed at older pedestrians. 


\section{APPENDIX A \\ SUMMARIES OF NHTSA PEDESTRIAN AND BICYCLIST SAFETY RESEARCH PROJECTS}

This section contains summaries of the pedestrian and bicyclist safety research reports sponsored by the Traffic Safety Programs' Office of Research and Technology and its predecessor organizations during the 1969-2007 period. The standard format gives the following information for each report/program, as appropriate:

- Title - authors, title, report number and date;

- Problem examined;

- Age group(s) addressed;

- Crash type(s) involved - original crash types (see appendix for crash descriptions)

- Abstract; and

- Related NHTSA research.

The reports are grouped under the headings listed below. Many of the studies could be assigned to more than one of the categories.

- PROBLEM IDENTIFICATION

Urban Pedestrian Crashes

Rural/Suburban Pedestrian Crashes

Bicycle Crashes

1990s Pedestrian and Bicycle Update

- NEW TECHNOLOGY/TOOLS

- PROGRAM REVIEWS AND SYNTHESIS

- PUBLIC AWARENESS

- CONSPICUITY/VISIBILITY

- COUNTERMEASURES

Integrated

Training

Messages

Regulations

Bicycle Helmet Promotions

Enforcement

Engineering

- ALCOHOL

- SPEED

- CHILDREN

Preschool

School Age - Urban

School age-Rural

- OLDER (65+) ADULTS 


\title{
PROBLEM IDENTIFICATION
}

\section{Urban Pedestrian Crashes}

Title: Snyder, M.B., \& Knoblauch, R.L. Pedestrian Safety: The Identification of Precipitating Factors and Possible Countermeasures. Final Report, Volumes I, II (Appendices), DOT HS 800 403 and DOT HS 800 404, January 1971. Washington, DC: National Highway Traffic Safety Administration.

Problem Examined: Why do pedestrian crashes occur and how can they be prevented?

Age Group(s) Addressed: All ages

Crash Type(s) Involved: All urban pedestrian crash types

\begin{abstract}
The study objective was to identify causes and countermeasures relevant to pedestrian crashes. Behavioral and descriptive data was collected by interviews and on-scene observations for over 2,000 pedestrian crashes in 13 major cities. Subsequent analyses emphasized individual case causation and crash type classification relevant to countermeasure implementation. Cases were divided into crash types on the basis of causal factors and target groups, to provide a basis for countermeasure identification. The five most frequent types accounted for over $50 \%$ of the sample cases. These crash types were: dart-out first-half, dart-out second-half, intersection dash, multiple threat, and vehicle turn/merge. Countermeasures relevant to each crash type are discussed. Volume II, Appendices, contains more detailed documentation of the findings.
\end{abstract}

Related NHTSA Research: Knoblauch, R.L. Causative Factors and Countermeasures for Rural and Suburban Pedestrian Accidents: Accident Data Collection and Analysis. Final Report, DOT HS 802 266, March 1977.Washington, DC: National Highway Traffic Safety Administration.

A landmark study that extended the crash typing methodology to the rural/suburban area.

Knoblauch, R.L., Moore, W., Jr., Schmitz, P.R., \& Sommers, B.J. Causative Factors and Countermeasures for Rural and Suburban Pedestrian Accidents: Accident Data Collection and Analysis - Appendices. Final Report, DOT HS 802 474, June 1977. Washington, DC: National Highway Traffic Safety Administration.

Contains the data collection forms and various data distributions. 
Title: Knoblauch, R.L. Urban Pedestrian Accident Countermeasures Experimental Evaluation: Volume II - Accident Studies. Final Report, DOT HS 801 347, February 1975. Washington, DC: National Highway Traffic Safety Administration.

Problem Examined: Once pedestrian crash types had been developed, there was a need for a database organized in terms of the types. This study was the beginning of a crash-type-specific database.

Age Group(s) Addressed: All ages

Crash Type(s) Involved: All pedestrian crash types

Abstract: A pedestrian crash data collection system was established in six major cities. The system involved using the regular police crash report form and a specifically designed supplementary data form. The information on the forms was combined, and the precipitating and predisposing factors, as well as the distribution of crash types in the crash database, were determined. Descriptive data on 2,044 pedestrian crashes from the six study cities is presented.

Related NHTSA Research: Snyder, M.B., \& Knoblauch, R.L. Pedestrian Safety: The Identification of Precipitating Factors and Possible Countermeasures. Final Report, Volumes I, II (Appendices), DOT HS 800403 and DOT HS 800 404, January 1971. Washington, DC: National Highway Traffic Safety Administration.

First study to identify pedestrian crash types. 
Title: Vayda A., \& Crespi, I. Public Acceptability of Highway Safety Countermeasures. Volume IV: Pedestrian Safety. Final Report. DOT HS 805 973, June 1981. Washington, DC: National Highway Traffic Safety Administration.

Problem Examined: What are the public's attitudes toward proposed highway safety countermeasures in the pedestrian safety area?

Age Group(s) Addressed: All ages

Crash Type(s) Involved: Dart-out, ice cream vendor, intersection dash

\begin{abstract}
This volume is part of a larger study providing information about public attitudes towards proposed highway safety countermeasures in three program areas: alcohol and drugs, unsafe driving behaviors, and pedestrian safety. Pedestrian safety countermeasures discussed in this volume include street safety classes for children, vendor regulations, and model parking laws. For the general public survey, acceptability issues are analyzed in terms of demographic characteristics (including age of children under 25), role of schools in safety training, perceived seriousness of the safety problem, and perceived effectiveness of the proposed countermeasure. Special interest perspectives include discussions of parental involvement in children's safety programs, implementation costs, significance of the safety problem, degree of inconvenience imposed on the public, and effectiveness in crash reduction.
\end{abstract}

The survey results suggested that drivers as well as nondrivers were highly receptive to plans for increasing the safety of pedestrians, especially children. Most of the drivers surveyed responded favorably to all of the pedestrian countermeasures. Safety training for children and prohibiting parking near crosswalks were especially acceptable, each of which received support from $88 \%$ of the drivers. Angle parking and the model vendor law ranked somewhat lower in acceptability than the other two pedestrian countermeasures, receiving support, respectively, from $81 \%$ and $72 \%$ of the drivers surveyed. 


\title{
PROBLEM IDENTIFICATION
}

\section{Rural/Suburban Pedestrian Crashes}

Title: Knoblauch, R.L. Causative Factors and Countermeasures for Rural and Suburban Pedestrian Accidents: Accident Data Collection and Analysis. Final Report, DOT HS 802 266, March 1977. Washington, DC: National Highway Traffic Safety Administration.

and

Title: Knoblauch, R.L., Moore, W., Jr., Schmitz, P.R., \& Sommers, B.J. Causative Factors and Countermeasures for Rural and Suburban Pedestrian Accidents: Accident Data Collection and Analysis - Appendices. Final Report, DOT HS 802 474, June 1977. Washington, DC: National Highway Traffic Safety Administration.

Problem Examined: In 1971 a landmark study identified pedestrian crash types for urban areas. This study describes a comparable effort for rural and suburban populations.

\section{Age Group(s) Addressed: All ages}

\section{Crash Type(s) Involved: All pedestrian crash types}

\begin{abstract}
The objectives of this study were to collect and analyze data on rural (non-urban) pedestrian crashes and to identify potential countermeasures. Data on a stratified random sample of over 1,500 rural and suburban crashes from six States was collected during interviews and onsite observations. These data included behavioral sequence items, site characteristics items and exposure data items directed at identifying the precipitating and predisposing causal factors in each crash. The data analysis emphasized the development of characteristic crash situations or "crash types" from groups of behaviorally-similar crashes. Although 23 crash types were identified, the 6 most frequently encountered types accounted for over $60 \%$ of the sample. These 6 included: walking along the roadway, dart-out first-half, dart-out second-half, midblock dash, intersection dash, and disabled vehicle related. Countermeasures intended to apply to each crash type are discussed.
\end{abstract}

Related NHTSA Research: Snyder, M.B., \& Knoblauch, R.L. Pedestrian Safety: The Identification of Precipitating Factors and Possible Countermeasures. Final Report, Volumes I, II (Appendices\}, DOT HS 800403 and DOT HS 800 404, January 1971. Washington, DC: National Highway Traffic Safety Administration. The first study to develop crash types in urban areas. 


\title{
PROBLEM IDENTIFICATION
}

\section{Bicycle Crashes}

Title: Cross, K.D., and Fisher, G. A Study of Bicycle/Motor-Vehicle Accidents: Identification of Problem Types and Countermeasure Approaches. 3 Volumes. Final Report, DOT HS 803 315, DOT HS 803316 (Appendices), DOT HS 803317 (Coding Index), September 1977.

Washington, DC: National Highway Traffic Safety Administration.

Problem Examined: A seminal study that undertook to identify bicycle/motor vehicle crash types and potential countermeasure approaches.

Age Group(s) Addressed: All age groups

Crash Type(s) Involved: All bicyclist crash types

\begin{abstract}
The purpose of this study was to determine the causes of bicycle/motor-vehicle crashes and to use data on crash causation to identify potential countermeasure approaches. Data were collected by interviews and on-site investigations for 753 nonfatal crashes and 166 fatal crashes. The sampling areas, each consisting of several contiguous counties, were located in California, Colorado, Florida, and Michigan. In addition to an analysis of descriptive data, crash cases were classified into "problem types" based upon traffic context, crash causes, and target groups. A total of 36 unique problem [crash] types were identified; the 10 most frequent problem types accounted for $67 \%$ of the fatal cases and $64 \%$ of the nonfatal cases. The results of the analyses of descriptive data are discussed and the distinguishing characteristics of each problem type are described. Potential countermeasure approaches are suggested for each problem type.
\end{abstract}

Volume II contains specimens of the various instruction manuals, questionnaires, and other datacollection instruments used in this study. It also contains a number of data tables which support the various graphs and summary tables presented in Volume I. Volume III is a coding index which describes the manner in which each data item was encoded. This volume of the report would be useful only to persons who have access to the raw data file and wish to use it to perform additional analyses.

Related NHTSA Research: Blomberg, R.D., Leaf, W.A., Hale, A., Farrell, M.L., \& Cross, K.D. Identification and Development of Countermeasures for Bicyclist/Motor-Vehicle Problem Types Volume I-Method and Training Program Descriptions, Final Report, DOT HS 806 326, August 1982. Washington, DC: National Highway Traffic Safety Administration.

Developed three prototype training countermeasures for selected bicyclist problem types.

Blomberg, R.D., Leaf, W.A., Hale, A., Farrell, M.L., \& Cross, K.D. Identification and

Development of Countermeasures for Bicyclist/Motor-Vehicle Problem Types Volume II-Public Information and Education Messages, Final Report, DOT HS 806 327, August 1982.

Washington, DC: National Highway Traffic Safety Administration.

Developed 15 prototype PI\&E messages for selected bicyclist problem types. 
Blomberg, R.D., Leaf, W.A., Hale, A., Farrell, M.L., \& Cross, K.D. Identification and Development of Countermeasures for Bicyclist/Motor-Vehicle Problem Types Volume III Model Regulations, Final Report, DOT HS 806 328, August 1982. Washington, DC: National Highway Traffic Safety Administration.

Developed eight prototype model traffic safety regulations for selected bicyclist problem types.

Blatt, J., \& Dueker, R.L. Assessment of the Safety-Relevance of Pedestrian and Bicyclist Programs. Volume I: Conduct and Results. Final Report. DOT HS 806 436, April 1983. Washington, DC: National Highway Traffic Safety Administration.

Provides instrument for assessing the safety relevance of bicyclist safety education programs.

Casey, S.M., Cross, K.D., Leaf, W.A., \& Blomberg, R.D. Bicyclists' Inclination and Ability to Search Behind Before Turning Left. Interim Report, DOT HS 805 893, February 1980.

Washington, DC: National Highway Traffic Safety Administration.

Investigated the effect of bicyclist searching rearward on bicyclist crashes.

Problem Examined: Research identified a type of crash in which a bicyclist makes a left-hand turn without searching to the rear or signaling and is struck by an overtaking motor vehicle. In order to develop countermeasures for this crash type, it was necessary to determine the willingness and ability of bicyclists to search rearward before making left-hand turns.

Age Group(s) Addressed: Children and adults

Crash Type(s) Involved: Bicyclist unexpected left turn

Abstract: It was necessary to understand the frequency with which bicyclists actually search behind before turning left, and to determine the ability of bicyclists to maintain lateral stability when looking behind. Hence, two separate studies were conducted.

The first, a field-observation study, was performed to assess the frequency with which bicyclists search behind before initiating a left-hand turn. Field investigators observed and recorded data on 1,012 left-hand turns by bicyclists. The relationships between search failure and selected environmental, operator and vehicular factors are described. The percentages of search failures were found to range from $23 \%$ at locations with high traffic density to $79 \%$ at locations with the lowest traffic density.

The second experiment measured the magnitude of the inadvertent lateral deviations that accompany a rearward search as a function of the bicyclist's age and riding experience. Of major interest was the magnitude of the largest deviation from a straight line path ("maximum error"). It was found that maximum error was greater for trials involving rearward search than for those which did not. However, the absolute error was relatively small. The 99th percentile error was only 20 inches. Error was lower for more experienced bicycle riders but was unrelated to age.

The totality of results indicates that it would be productive to promote universal rearward searches in bicycle safety education or training programs. 
Related NHTSA Research: Cross, K.D., \& Fisher, G. A Study of Bicycle/Motor-Vehicle Accidents: Identification of Problem Types and Countermeasure Approaches. 3 Volumes. Final Report, DOT HS 803 315, DOT HS 803316 (Appendices), DOT HS 803 317, September 1977. Washington, DC: National Highway Traffic Safety Administration.

Provides basic information on bicyclist crash types. 


\title{
PROBLEM IDENTIFICATION
}

\section{0s Pedestrian and Bicycle Update}

Title: Hunter, W.W., Stutts, J.C., Pein, W.E, \& Cox, C.L. Pedestrian and Bicycle Crash Types of the Early 1990s. Publication No. FHWA-RD-95-163, 1995. Washington, DC: Federal Highway Administration.

Problem Examined: Need for simplified pedestrian and bicycle crash typing schemes.

Age Group(s) Examined: All ages

Crash Type(s) Involved: All crash types

\begin{abstract}
The purpose of this research sponsored by FHWA was to apply the basic NHTSA pedestrian and bicyclist typologies to a sample of recent crashes, and to refine and update the crash-type distributions, paying particular attention to roadway and location factors. The specific study aims were to: (1) identify and code, according to NHTSA typologies, a recent sample of approximately 5,000 pedestrian-motor vehicle and 3,000 bicycle-motor vehicle crashes, (2) determine the specific roadway, location, and other factors associated with each crash type and (3) identify situations where engineering and/or educational or regulatory countermeasures might be employed effectively to reduce the frequency of pedestrian or bicycle crashes. The final project report was a descriptive study identifying situations under which pedestrian and bicyclist crashes with motor vehicles take place. The report lays the groundwork for the implementation of engineering, educational, and regulatory countermeasures to reduce the number of such crashes.

Related NHTSA Research: Snyder, M.B., \& Knoblauch, R.L. Pedestrian Safety: The Identification of Precipitating Factors and Possible Countermeasures. Final Report, Volumes I, II (Appendices), DOT HS 800403 and DOT HS 800 404, January 1971. Washington, DC: National Highway Traffic Safety Administration.

First study to identify pedestrian crash types.

Cross, K.D., \& Fisher, G. A Study of Bicycle/Motor-Vehicle Accidents: Identification of Problem Types and Countermeasure Approaches. 3 Volumes. Final Report, DOT HS 803 315, DOT HS 803316 (Appendices), DOT HS 803 317, September 1977. Washington, DC: National Highway Traffic Safety Administration.

Provides basic information on bicyclist crash types.
\end{abstract}




\title{
TECHNOLOGY / TOOLS
}

Title: Brown, R., \& Sutherland, L.C. An Audible Automobile Back-Up Pedestrian Warning Device - Development and Evaluation. Final Report. DOT HS 802 083. November 1976.

Washington, DC: National Highway Traffic Safety Administration.

Problem Examined: Can an effective back-up warning signal be developed to prevent pedestrian crashes?

Age Group(s) Addressed: All age groups

Crash Type(s) Involved: Backing vehicle

\begin{abstract}
The purpose of this study was to develop and field-test an audible back-up warning device for use on automobiles. Detailed criteria of pedestrian age and hearing ability combined with noise characteristics of typical crash sites provided the basis for selection of a warning signal format. The warning signal (a tone at $1250 \mathrm{~Hz}$ pulsed on for $0.1 \mathrm{sec}$ and off for $0.2 \mathrm{sec}$ ) was generated by a small loudspeaker mounted at the rear of the vehicle. An essential element of the design, that the system sense the ambient level and adjust its output accordingly, results in a warning signal level approximately equal to the A-weighted noise level throughout the danger zone. This is comparable to a level at least $10 \mathrm{~dB}$ above the pedestrian's detection threshold.

Evaluation of a prototype system was conducted in typical parking sites using pedestrian subjects of opportunity. The age distribution of the subjects was fairly equivalent to the age distribution of pedestrian victims of back-up crashes. Results comparing the normal situation with a test sequence using the warning signal indicated a tenfold improvement in the number of pedestrians warned of the presence of a backing vehicle.
\end{abstract}


Title: Blatt, J., \& Dueker, R.L. Assessment of the Safety-Relevance of Pedestrian and Bicyclist Programs. Volume I: Conduct and Results. Final Report. DOT HS 806 436, April 1983. Washington, DC: National Highway Traffic Safety Administration.

Problem Examined: Over the years, NHTSA has been asked by State and local safety practitioners to provide information on the suitability of various pedestrian and bicyclist safety education programs. Generally, a comprehensive and defendable set of criteria against which such products can be judged was not available. This project was designed to develop a package of material to be used in assessing the safety relevance of pedestrian and bicyclist safety education programs.

Age Group(s) Addressed: Primarily children, but also applicable to adults

Crash Type(s) Involved: All pedestrian and all bicyclist crash types

Abstract: This document (Volume One of a Two-Volume Report) describes the development of a paper-and-pencil instrument for assessing the safety relevance of pedestrian and bicyclist safety education programs. The safety relevance of the program is the extent to which its content reflects the findings of NHTSA research in crash causation and countermeasure development. This instrument, called the Program Assessment Kit (PAK), was developed in response to a need to provide Federal, State, and local safety program personnel with a comprehensive and systematic means for performing comparative evaluation of alternate programs. It can also be used as an aid to the improvement of existing programs and the development of new programs.

The PAK was developed using the Worth Assessment Technique. Assessment areas and weights were provided by experts in pedestrian safety, bicycle safety and education. The PAK contains a set of Program Assessment Scales (PAS) for both program types by various program age levels. Each PAS provides 11 subscores and three area scores - safety relevance (content), instructional approach and adequacy of the material. It also includes a checklist survey of implementation considerations, guidelines for interpreting reported program effectiveness and a description of NHTSA pedestrian and bicyclist crash types.

The PAK was tested by having project staff independently use the PAK to assess a sample of 23 pedestrian and bicyclist safety education programs. High inter-rater reliabilities were obtained (mean of .843) and all sub and area scores except two ranged between .657 and .905. These exceptions were the result of ambiguous instructions which were subsequently revised. The mean composite (overall) score for the pedestrian programs sampled was 37.5 (100 points possible); for bicyclist programs, 42.8 . The most common program weaknesses identified were too much emphasis on irrelevant information (thus reducing the time or emphasis available for safety relevant content) and too little practice of safety behaviors.

Volume One also contains a listing and classification of 97 non-NHTSA programs developed in the United States which were available as of May 1981.Volume Two of the report contains the PAK in ready-to-use form. 
Related NHTSA Research: Snyder, M.B., \& Knoblauch, R.L. Pedestrian Safety: The Identification of Precipitating Factors and Possible Countermeasures. Final Report, Volumes I, II (Appendices\}, DOT HS 800403 and DOT HS 800 404, January 1971. Washington, DC:

National Highway Traffic Safety Administration.

Basic study for information regarding urban pedestrian crash types.

Knoblauch, R.L. Causative Factors and Countermeasures for Rural and Suburban Pedestrian Accidents: Accident Data Collection and Analysis. Final Report, DOT HS 802 266, March 1977. Washington, DC: National Highway Traffic Safety Administration.

Basic study for information regarding rural/suburban pedestrian crashes.

Cross, K.D., \& Fisher, G.A. A Study of Bicycle/Motor-Vehicle Accidents: Identification of Problem Types and Countermeasure Approaches. Volumes I and II, Final Reports, DOT HS 803 315 and DOT HS 803 316, September 1977. Washington, DC: National Highway Traffic Safety Administration.

Basic study for information regarding bicycle crash types. 
Title: Rose, A. M., Levine, J. M., \& Eisner, E.J. Measurement of Pedestrian Behavior. Final Report, DOT HS 802 105, November 1976. Washington, DC: National Highway Traffic Safety Administration.

Problem Examined: This study emphasizes the capability of a countermeasure to modify pedestrian or driver behaviors implicated in pedestrian crashes. It examines a complete range of behaviors and measurement systems involved in assessing countermeasure effectiveness.

\title{
Age Group(s) Addressed: All ages
}

Crash Type(s) Involved: Dart-out, intersection dash, vehicle turn/merge, pedestrian hits vehicle, multiple threat, bus stop related, ice cream vendor, backing vehicle, working in road, freeway crossing

\begin{abstract}
The most direct approach to countermeasure evaluation would involve implementing a countermeasure at selected sites and comparing pre- and post-countermeasure crash rates with those obtained at control sites. This approach uses crash rate as the criterion of effectiveness; however, it is highly inefficient and costly since years of monitoring the relatively low-frequency crash event are likely to be required before sufficient information can be accumulated to permit meaningful conclusions to be reached. In light of this and in line with the goal of determining potential countermeasure effectiveness prior to full-scale or widespread implementation, a supplementary approach to evaluation has been developed. This approach emphasizes the capability of a countermeasure to modify critical pedestrian and driver behaviors presumed to relate to various types of crashes. It was assumed that an assessment of the capability of countermeasures to modify selected critical behaviors would permit conclusions to be drawn regarding the potential effectiveness of the countermeasures for crash reduction.
\end{abstract}

A categorization of behavioral items was developed which included only search and locomotion behaviors, since these are the only observable events in the crossing situation. There are five parameters of pedestrian searching behavior: object, direction, duration, sequence, and position. These terms refer to, respectively, the pedestrian's object(s) of attention while searching, what direction they look in (with respect to their direction of movement), how long they look in each direction, the sequence of directional searches, and their position when searching (in terms of both distance between pedestrian and curb and between pedestrian and approaching vehicle). There are four parameters of pedestrian locomotion: velocity, acceleration, direction (with respect to the curb), and position (again in terms of both distance between pedestrian and curb and between pedestrian and approaching vehicle). Parameters concerned with driver behavior are essentially equivalent to pedestrian parameters. The five search parameters of object, duration, direction, sequence, and position are exactly parallel for the driver and the pedestrian. Locomotion parameters include vehicle movement characteristics (vehicle path and speed) and driver control characteristics (velocity and direction).

Judgments were made as to which of the behavioral parameters were likely to be significantly impacted upon given the implementation of each of 24 potential countermeasures. These judgments were formulated for each of 11 selected crash types. The result of this procedure was 
the determination of a set of behaviors which were presumed to be most important to measure for the purpose of evaluating the effectiveness of a countermeasure on a specific crash type.

Eleven measurement systems were evaluated in terms of their cost effectiveness in measuring each of the behavioral parameters. These systems included direct observation, interview, road tubes, radar, three types of filming systems, and four types of television systems. Effectiveness was assessed along six dimensions. These were validity, reliability, accuracy, ease of implementation, efficiency, and environmental range. A total system effectiveness index was computed as the product of the ratings of a system on each dimension. Five cost components were identified which constituted the total cost of system use. These were purchase price, implementation, maintenance, operation, and data reduction costs. The ratio of system effectiveness to total cost was computed for each system as it applied to the measurement of each of the behavioral parameters. The result was a set of data which can be used to select the most cost-effective measurement system to employ in order to measure a particular behavioral parameter. Further, the methodology and procedures developed can be used to generate costeffectiveness information for other measurement systems which were not evaluated in the present effort. 
Title: Walk Alert - A National Pedestrian Safety Program Guide. 1994

Problem Examined: How to make community leaders aware of what they can do to protect their community from preventable injury and death from pedestrian crashes.

Age Group(s) Addressed: All ages

Crash Types Involved: All pedestrian crash types

Abstract: To promote safe walking and to reduce the number of pedestrian traffic crashes, the FHWA, NHTSA, the American Automobile Association (AAA), the National Safety Council, and more than 100 service organizations and community groups across the nation joined together to create a national pedestrian safety program called Walk Alert. Because crashes that involve pedestrians often have serious consequences, the program takes a thorough and comprehensive look at crash prevention. In defining its comprehensive approach, Walk Alert examines the three components in any community that affect pedestrian safety: education, engineering, and enforcement. Since the behavior of drivers and pedestrians is a major factor in both causing and preventing crashes, Walk Alert focuses more on educating pedestrians and drivers about what they can do to reduce the risk of crashes. The role of traffic engineering is to develop and refine the physical facilities and space - roadways, sidewalks, crosswalks, lights, and traffic control devices - that help safeguard pedestrians. Legislation provides regulations necessary for ensuring that both pedestrians and drivers share responsibilities for pedestrian safety. Law enforcement agencies maintain crash records, investigate crashes, and protect pedestrians and motorists alike by enforcing regulations on sharing the roadway.

The Walk Alert program contains information on how to develop, implement, sustain, and evaluate a pedestrian safety program. It also includes safety messages for pedestrians of all ages, a pedestrian safety checklist, sample publicity material, and information on other resources available. 
Title: Harkey, D.L., Mekemson, J., Chen, M., \& Krull, K.A. Pedestrian and Bicycle Crash Analysis Tool (PBCAT) Software and User's Manual. FHWA-RD-99-192, June 2000.

Washington, DC: Federal Highway Administration.

Problem Examined: How can crash types and countermeasures for them be easily identified?

Age Group(s) Addressed: All ages

Crash Types Involved: All pedestrian and bicyclist crash types

Abstract: FHWA and NHTSA supported the development of PBCAT through the University of North Carolina's Highway Safety Research Center. PBCAT is a software product intended to assist State and local bicycle coordinators, planners, and engineers. PBCAT accomplishes this goal through the development and analysis of a database containing details associated with crashes between motor vehicles and pedestrians or bicyclists. One of these details is the crash type which describes the pre-crash actions of the parties involved.

Pedestrian and bicycle crashes are a serious problem. Approximately 6,500 pedestrians and 900 bicyclists are killed each year, which is $16 \%$ of all traffic fatalities. An additional 90,000 pedestrians and 60,000 bicyclists are reported to be injured as a result of collisions with motor vehicles. Crash typing can help define the problem. The development of effective countermeasures to help prevent bicyclist and pedestrian crashes is hindered by insufficient detail on computerized State crash files. Analysis of this data can provide information on where pedestrian and bicyclist crashes occur (city, street, intersection, two-lane road, etc.), when they occur (time of day, day of week, etc.), and characteristics of the victims involved (age, gender, injury severity, etc.). This data cannot provide a sufficient level of detail regarding the sequence of events leading to the crash.

The crash typing methodology included in PBCAT allows the user to quickly determine the crash type through a series of on-screen questions about the crash and the maneuvers of the parties involved. PBCAT is adaptable and easy to use. It enables practitioners to generate valuable information for promoting bicycle and pedestrian safety and for designing safer facilities where bicyclists, pedestrians, and motor vehicles interact. The software has the following features:

- Ability to customize the database in terms of variables, units of measurement, and location referencing.

- Ability to import/export data from/to other databases.

- Ability to produce a series of tables and graphs defining the various crash types and other factors associated with the crashes such as age, sex, light conditions, etc.

- Recommended countermeasures linked to specific bicycle and pedestrian crash types.

- Resource and reference information related to specific countermeasures.

- User-friendly on-line instructions and help features along with a user's manual. 
Title: Dunlap and Associates, Inc. Bicycle Safety Resource Guide. NHTSA, 1998. Washington, DC: National Highway Traffic Safety Administration.

Problem Examined: How to identify countermeasures that may be used by specific implementers to help solve various bicyclist safety problems.

Age Group(s) Addressed: All ages

Crash Types Involved: All bicyclist crash types

\begin{abstract}
The Bicycle Safety Resource Guide was prepared for the bicycle safety professional and others who are proactive in developing programs at the State or community level. It provides a compilation of existing and proposed countermeasures that can be used by a variety of implementers to help solve a wide range of bicycle safety problems.
\end{abstract}

The primary objective of the study was to analyze the current bicycle safety problem and develop countermeasures to impact that problem. Contacts with numerous bicycle safety professionals revealed that many countermeasures have been developed and much work has been done on bicycle/motor vehicle crash types, but little has been done to simplify and operationalize the relationship between problems and remedial actions. It was decided that people responsible for bicycle safety at the State and local levels could benefit from the availability of a comprehensive resource guide that identified countermeasures that could be carried out by different implementers. A guide was therefore developed that identified 40 different bicycle problem areas and the countermeasures that could be used by 15 different implementers to help reduce each problem area. Where no countermeasures were located, ideas for countermeasure development were included in the guide. Therefore, the bicycle safety professional can enter the guide with a problem area and identify existing or proposed countermeasures that can be used to help combat that problem by each of the 15 implementers. In all, 226 existing and 75 proposed countermeasures were described. One of the proposed countermeasures (a media guide) was also fully scripted. The resultant Bicycle Safety Resource Guide was produced in CD-ROM format.

As part of the study, two separate analyses were conducted. One revealed that the Cross and Fisher crash types identified in the 70s and subsequently improved by NHTSA and FHWA were still valid. The second analyzed the role of alcohol in bicycle safety and concluded that it is a serious problem. In an analysis of FARS data, BACs were found to be positive in $33 \%$ of age $15+$ victims who were tested for alcohol and in $21 \%$ of the total fatal sample. In an analysis of essentially nonfatal data available from the Highway Safety Research Center, BACs were estimated to be positive in $22 \%$ of age $15+$ crashes.

The guide was prepared for the National Highway Traffic Safety Administration and Federal Highway Administration under subcontract between Dunlap and Associates, Inc., and the Highway Safety Research Center, University of North Carolina, as part of Task Order 9 of Federal Highway Administration Contract DTFH61-92-C-00138, Development and Test of Bicycle Safety Countermeasures. 
Related NHTSA Research: Dunlap and Associates, Inc. Bicycle and Pedestrian Safety Resource Guide, DOT HS 809 977, 2006. Washington, DC: National Highway Traffic Safety Administration. 
Title: Dunlap and Associates, Inc. Bicycle and Pedestrian Safety Resource Guide, DOT HS 809 977, 2006. Washington, DC: National Highway Traffic Safety Administration.

Problem Examined: How to identify countermeasures that may be used by specific implementers to help solve various pedestrian and bicyclist safety problems.

Age Group(s) Addressed: All ages

Crash Types Involved: All pedestrian and bicyclist crash types

\begin{abstract}
NHTSA sponsored the development of resource guides of both bicycle and pedestrian safety material. The bicycle guide (sponsored also by FHWA) was produced in CD-ROM format in 1998 under a contract between Dunlap and Associates, Inc. and the University of North Carolina's Highway Safety Research Center. It was designed to aid the bicycle safety professional in matching problems with countermeasures that can be used to attack those problems. It included existing countermeasures and ideas for countermeasures that might be developed to help solve specific highway safety problems. The resource guide had three major dimensions: problem areas, implementers and countermeasure format/use. The highway safety professional could initiate a search on any one of the three dimensions to identify countermeasures that are appropriate for specific problems and specific implementers. In addition, the searcher could use the hyperlinks to obtain specific information on specific topics. The guide was widely distributed and well received. The pedestrian guide was prepared in the same format in 2002 but was not formally produced or distributed. With the ever-increasing production of new safety material for both pedestrians and bicyclists, both guides became out-ofdate. NHTSA therefore asked Dunlap and Associates to update both guides and combine them in a single CD-ROM. This effort involved contacts with all known producers of safety material to confirm the availability of previously described material and to obtain information on new products It resulted in a CD-ROM that combined countermeasure information for both pedestrian and bicyclist safety.
\end{abstract}

Related NHTSA Research: Dunlap and Associates, Inc. Bicycle Safety Resource Guide. NHTSA, 1998. Washington, DC: National Highway Traffic Safety Administration. 
Title: Blomberg, R.D., \& Cleven, A.M.. Resource Guide on Laws Related to Pedestrian and Bicycle Safety. DOT HS 809 368, 2000. Washington, DC: National Highway Traffic Safety Administration.

Problem Examined: This study was designed to create a database of existing and model laws that may affect pedestrian and bicyclist safety.

Age Group(s) Addressed: All ages

Crash Type(s) Involved: All pedestrian and bicyclist crash types

\begin{abstract}
Dunlap and Associates, Inc., developed a Resource Guide on Laws Related to Pedestrian and Bicycle Safety. This guide contains a compilation of vehicle and traffic laws that were judged by the guide's developers to have the potential to affect pedestrian or bicycle safety, either positively or negatively. The guide was designed for easy use by anyone interested in vehicle and traffic law and pedestrian or bicycle safety. This might include State and local bicycle and pedestrian professionals, legislative service bureaus, and others who work with bicycle and pedestrian laws. It can be used to select laws that enhance pedestrian or bicycle safety, to assess a State's position with respect to other States or the state of the art or to examine the extent to which the prevailing vehicle and traffic law may impact the generation of pedestrian or bicycle crashes, particularly those with motor vehicles.
\end{abstract}

Created in CD-ROM format, the guide presents a selection of key vehicle and traffic law provisions drawn from three sources-the Uniform Vehicle Code prepared by the National Committee on Uniform Traffic Laws and Ordinances; other State laws and local ordinances that are perceived to have a positive effect on pedestrian or bicycle safety; and model laws prepared or adapted by this project to meet specific safety needs. The safety relevance of each key provision is assessed in terms of its likely effects on the causes of bicycle or pedestrian crashes with motor vehicles, the prevention or reduction of bicyclist or pedestrian injuries and possible effects on pedestrian and bicycle injury-producing situations that do not involve motor vehicles, e.g., falls. Each key provision is cross-referenced to a description of how that concept is implemented in the 50 States. The guide is an easily accessed database of current and proposed laws that may affect pedestrian and bicycle safety. 
Title: Hanowski, R.J., Spaulding, J.M., Gaskins, C., Schaudt, W.A., Miller, S., Holbrook, T., Olson, R.L., Dingus, T.A., Hickman, J.S.,Huey, R., \& Llaneras, E.E. Field Evaluation of Alternative Automated Systems for Reducing Illegal Passing of School Buses. Final Report, awaiting release. Washington, DC: National Highway Traffic Safety Administration.

Problem Examined: Can an automated system be developed to detect vehicles that illegally pass stopped school buses and record their license plates and drivers?

Age Group(s) Addressed: Ultimately, safety of the school-age child

Crash Type(s) Involved: School bus related

Abstract: Research has shown that approximately $60 \%$ of fatalities that occur in school bus loading and unloading zones were the result of motorists who failed to stop when the vehicle's stop-arm was extended and the red warning lights were flashing. To address this problem, NHTSA sponsored research aimed at developing an automated system for detecting and recording the license plates and drivers of vehicles that illegally pass stopped school buses. The study was conducted in two phases: initial system research and system refinement. The initial development effort was co-conducted by the Virginia Tech Transportation Institute and WESTAT. Refinement of the system was accomplished by VTTI.

Analyses were conducted to assess the technical, administrative and legal feasibility of automated enforcement systems for this application. A key output of the feasibility analyses was a set of general recommended design specifications for an initial prototype system as follows: five cameras (two 110-degree low-resolution cameras for violation detection and image subtraction and three $640 \times 480$ high-resolution cameras for capturing front and rear license plates as well as a face view of the driver), a computer with three video channels and a video buffer, an infrared pulse source (to illuminate the driver's face in low-light conditions, batteries (or hardwire to the bus' on-board battery) and recording of pertinent non-video violation information (e.g., date, time, etc.). The system was automatically activated once the vehicle's amber lights were switched on. Camera images were continuously processed upon system activation, but images were recorded onto the computer only when a violation occurred. This was achieved by buffering the video images in memory. Pertinent non-video information (date and time, etc.) was recorded in a data file that was linked to the video files.

Testing of the initial prototype revealed two primary limitations: it did not perform well enough at capturing driver facial images during the day and was not able to capture license plate images at night. These two limitations led to the development of the refined prototype system. The refined prototype system was contained entirely in a single housing (pod). It consists of five cameras - two facing forward, two facing rearward and one centrally located. The system has two high intensity discharge (HID) spotlights and one side-radar unit that is used to trigger the cameras. The HID spotlights replaced the infrared lights used in the initial prototype system. A side object detection system (SODS) radar replaced the front and rear cameras used for event triggering in the initial system. Ad hoc testing was conducted to determine whether or not the system functioned in certain conditions, e.g., could the system detect a vehicle passing the bus in the adjacent lane at $15 \mathrm{mph}$ ? The refinement stage also included an effort to design a conceptual 
pod that could be manufactured to hold the system components. Recommendations were made for system improvement and further testing. 
Title: Fell, J. C., \& Toth, G. R. Pedestrian Accidents: A State-of-the-Art 1970-1980. Technical Report, DOT HS 806 270, September 1981. Washington, DC: National Highway Traffic Safety Administration.

Problem Examined: Provides an overview of crash data in the pedestrian area for a 10-year period.

Age Group(s) Addressed: All ages

Crash Type(s) Involved: All pedestrian crash types

\begin{abstract}
This report discusses the pedestrian crash problem in this country by providing statistics on the basic characteristics of the crashes and summarizing the findings from four major research studies.
\end{abstract}

It appears that the age and sex of the pedestrian, the location of the crash, and the type of vehicle striking the pedestrian all play an important role in the problem. The pedestrian problem truly is most severe for the young ( 5 to 8 years old) and the old ( $>64$ years old) with males being overrepresented in all age groups but especially those pedestrians between 25 to 34 . Three location factors are very significant in fatal crashes - rural roads, high-speed roads, including major arteries, and non-intersection areas. The size of the striking vehicle is also important: the heavier the vehicle, the more likely a fatality.

In the United States experience, there have been four major pedestrian crash research studies in the 1970s which have had important results. These four studies have provided further detail and new findings in the areas of injury causation and severity, pedestrian crash topologies and scenarios, and the role of alcohol involvement. Findings and recommendations emanating from these studies are reported and discussed. Future research using the National Automotive Sampling System is recommended.

The report concludes that the pedestrian problem is unique and has no single, high impact solution. A concerted effort of several promising countermeasure approaches must be made to reduce that loss. 
Program Reviews and Synthesis

Title: Preusser, D. F., Leaf, W.A., DeBartolo, K.B., \& Blomberg, R.D. The Effect of Right-TurnOn-Red on Pedestrian and Bicyclist Accidents. Final Report, DOT HS 806 182, October 1981. Washington, DC: National Highway Traffic Safety Administration.

Problem Examined: To reduce the uncertainty concerning the effects of Right-Turn-On-Red on pedestrian and bicyclist crashes, and to provide quantitative and qualitative descriptions of any safety problems identified.

Age Group(s) Addressed: Adults and children

Crash Type(s) Involved: Intersection-related

Abstract: Right-Turn-On-Red in its "Western" or "permissive" version allows motorists to turn right on a red signal after stopping unless prohibited by a sign. Many States adopted Western RTOR in the mid-1970s. The objectives of this study were to assess the impact of adopting Western RTOR on the frequency of pedestrian and bicycle crashes with motor vehicles, and to determine the characteristics of any pedestrian and bicycle RTOR crashes. Data from the States of New York, Ohio, and Wisconsin and the cities of Los Angeles and New Orleans were examined. Time series was the major analytic technique for determining pre/post crash rate changes. Content analyses of police reports provided data on crash characteristics.

Measures of pedestrian and bicycle crashes involving a motorist making a right turn at a signalized location increased significantly at all study sites after the adoption of Western RTOR. Estimates of the magnitude of the increases ranged from $43 \%$ to $107 \%$ for pedestrian crashes and $72 \%$ to $123 \%$ for bicyclist crashes. Over half of the crashes in which a vehicle turned right at a signalized location after the adoption of Western RTOR involved a right turn on a red signal. These RTOR crashes constituted between one $\%$ and three $\%$ of all pedestrian or bicycle crashes in the studied locations. The majority of these RTOR crashes involved a driver looking left for a gap in traffic and striking a pedestrian or bicyclist coming from the driver's right. Educational countermeasures for bicyclists and pedestrians and traffic engineering approaches, including the further development of warrants for sign prohibitions of RTOR, appear to be worthy of additional research.

Related NHTSA Research: Compton, R.P., \& Milton, E.V. Safety Impact of Permitting RightTurn-On-Red: A Report to Congress By the National Highway Traffic Safety Administration. DOT HS 808 200, December 1994. Washington, DC: National Highway Traffic Safety Administration.

Provides an update on the RTOR and LTOR situations. 
Title: Ehrlich, P., Farina, A., Pavlinski, L., \& Tarrants, W.E. Effectiveness and Efficiencies in Pedestrian Safety. Technical Report, DOT HS 806 131, March 1982. Washington, DC: National Highway Traffic Safety Administration.

Problem Examined: At the urging of Congress, NHTSA and FHWA conducted an evaluation of various programs in the highway safety area. This report covers the area of pedestrian safety.

Age Group(s) Addressed: All ages

Crash Types Involved: All pedestrian crash types

\begin{abstract}
Pedestrian fatalities constitute $16 \%$ of total highway-related fatalities. Excluding motor vehicle occupants, pedestrians comprise the target single category of fatalities on the nation's streets and highways. A total of $85 \%$ of all pedestrian crashes and $60 \%$ of all pedestrian deaths occur on urban streets. In some large urban areas, 40 to $50 \%$ of the traffic deaths are pedestrians. Alcohol has been implicated with estimates of one of every three adult pedestrian crashes involving alcohol in urban areas. Poor visibility, or lack of conspicuity, has been identified as a contributing causal factor in a large number of nighttime and daytime pedestrian crashes. In 1981, pedestrian deaths numbered about 8,000 annually and pedestrian injuries almost 150,000 annually. The government's countermeasure focus has been on planning, design, construction and use of pedestrian facilities such as sidewalks, pathways, bridges and underpasses. Selected behavioral countermeasures include education, safety messages, model regulation, traffic engineering, and enforcement. Also included are programs for the elderly and handicapped, parents and pre-school children, and improved visibility.
\end{abstract}


Program Reviews and Synthesis

Title: National Highway Traffic Safety Administration. Pedestrian Injury Reduction Research. Report to the Congress, DOT HS 808 026, June 1993. Washington, DC: National Highway Traffic Safety Administration.

Problem Examined: To report on the research conducted by the agency to reduce deaths and injuries to pedestrians.

Age Group(s) Addressed: All ages

Crash Type(s) Involved: All pedestrian crash types

Abstract: NHTSA has prepared this report on the status of research efforts investigating the possibility of reducing pedestrian injuries and fatalities in response to the Senate Appropriations Committee's request that:

NHTSA submit a report on data it has collected and research conducted regarding ways of reducing pedestrian deaths and injuries through making vehicles more forgiving by removing sharp edges, softening the hood and cowl area, increasing the space between the hood and engine components, and other approaches, the cost of such designs in new cars, and on the numbers of deaths and injuries currently by type of injury and by vehicle type causing the injuries. The report shall include information on vehicle designs for pedestrian protection in other countries.

This report presents (1) highlights of research conducted to date to explore the technology and feasibility of modifying vehicle designs to better protect pedestrian impact victims, and (2) highlights of research and programs to avoid pedestrian-vehicle impacts through behavioral modification.

After an introduction, the section of the report dealing with vehicle changes that would lessen pedestrian fatalities and injuries presents information on head injury reduction, thoracic injury reduction, and leg injury reduction. The section of the report dealing with avoiding pedestrianvehicle impacts presents information on behavioral modification research and programs that NHTSA recommends to States and communities that are based on this research. 
Title: Compton, R.P., \& Milton, E.V. Safety Impact of Permitting Right-Turn-On-Red: A Report to Congress By the National Highway Traffic Safety Administration. DOT HS 808 200, December 1994. Washington, DC: National Highway Traffic Safety Administration.

Problem Examined: Updates the safety status of right-turn-on-red and left-turn-on-red laws in regard to pedestrian and bicyclist crashes.

Age Group(s) Addressed: All ages

Crash Type(s) Involved: Right-turning crashes at signalized intersections

\begin{abstract}
The Energy Policy Act of 1992 required NHTSA to conduct a study of the safety impact of permitting right and left turns on red lights. This report presents a brief summary of the current status of State implementation of laws permitting right and left turns at red lights, a brief review of previous research, and presents the results of analyses of currently available data assessing the safety impact of permitting right turns on red.
\end{abstract}

Related NHTSA Research: Preusser, D. F., Leaf, W.A., DeBartolo, K.B., and Blomberg, R.D. The Effect of Right-Turn-On-Red on Pedestrian and Bicyclist Accidents. Final Report, DOT HS 806 182, October 1981. Washington, DC: National Highway Traffic Safety Administration. NHTSA and FHWA's earlier study on the crash effects of RTOR. 
Title: Stutts, J.C., Hunter, W.W., Tracy, L., \& Wilkinson, W.C., III. Pedestrian and Bicyclist Safety: A Review of Key Program and Countermeasure Developments During the 1980s. Final Report, DOT HS 808 108, March 1992. Washington, DC: National Highway Traffic Safety Administration.

Problem Examined: This report chronicles events occurring in the pedestrian and bicyclist safety areas during a period when NHTSA activity was minimal.

Age Group(s) Addressed: All ages

Crash Type(s) Involved: All pedestrian and bicyclist crash types

Abstract: This report was prepared to review key countermeasure developments and program activities impacting on pedestrian and bicyclist safety over the past decade. Key national level policies and trends pertaining to pedestrians and bicyclists are highlighted to set the stage for the review. The remainder of the report is organized according to educational, engineering, and enforcement/regulatory program areas, and within each, national, State and local activities.

Pedestrian safety activity has been led by the Federal Government and has concentrated on the development of comprehensive program guides, and support material, with some continued funding for facility design and engineering countermeasure development and evaluation. There have also been recent efforts to work with local law enforcement agencies and to incorporate pedestrian safety into community traffic safety programs.

Bicycle countermeasure development and program activities have followed a very different path, led by national, non-government organizations such as the Bicycle Federation of America, the National Safe Kids Campaign, the American Academy of Pediatrics, and others. In contrast to pedestrian safety activities which have tended to follow a "top down" hierarchy, bicycle activities during this period have primarily been "grass roots" efforts. 


\section{PUBLIC AWARENESS}

Title: Partnership for a Walkable America. Contract No. DTFH61-92-C-00138

Problem Examined: The objective of this joint FHWA/NHTSA project is to increase awareness of pedestrian safety problems on a nationwide level in order to establish a base of support for current and future safety programs aimed at reducing these problems. It was realized early on that neither of the two agencies had the resources to mount large public information campaigns that might do the job. Networking with a host of other organizations was a necessary step.

Age Group(s) Addressed: All ages

Crash Type(s) Involved: Unspecified

Abstract: The contractor, the University of North Carolina's Highway Safety Research Center in association with Dunlap and Associates, the Pedestrian Federation of America, and the Center for Applied Research, created a new national organization - the Partnership for a Walkable America. The partnership was a public/private alliance committed to improving safety and increasing access for pedestrians while promoting the health benefits of walking. The three goals of safety, access, and health create a broad-based alliance that attracts a wide variety of organizations. The members were national government agencies and non-profit organizations concerned about three main areas: health, safety and the environment. Members of the steering committee of the alliance included the following: Pedestrian and Bicycle Information Center, NHTSA, FHWA, Centers for Disease Control and Prevention, Institute of Transportation Engineers, and the Robert Wood Johnson Foundation. The Partnership sponsored the International Walk to School Day Program, provided walkability and bikeability checklists and presented annual pedestrian project awards. The activities of the Partnership for a Walkable America have now been taken over by the Pedestrian and Bicycle Information Center-a clearinghouse sponsored by FHWA with support from NHTSA. 


\title{
CONSPICUITY / VISIBILITY
}

Title: Hale, A., \& Zeidler, P. Review of the Literature and Programs for Pedestrian and Bicyclist Conspicuity. Technical Report, DOT HS 806 564, April 1984.

Problem Examined: The topic of conspicuity - the ability to be seen - pervades the areas of pedestrian and bicyclist safety. This study provides a review of the world literature and describes conspicuity-enhancing programs.

Age Group(s) Addressed: All ages

\section{Crash Type(s) Involved: Unspecified}

\begin{abstract}
This report reviews literature bearing upon the problem of pedestrian and bicyclist conspicuity, and discusses the activities and accomplishments of various programs conducted to enhance the conspicuity of pedestrians and bicyclist. The results of a world-wide search for conspicuity relevant material are reported along with descriptions of national foreign programs to enhance pedestrian, bicyclist, and motorcyclist conspicuity. The level of discussion in this document is addressed to a reader with a background in the technical aspects of human vision in the highway setting.
\end{abstract}

Related NHTSA Research: Blomberg, R.D., Hale, A., and Preusser, D.F. Conspicuity for Pedestrians and Bicyclists: Definition of the Problem, Development and Test of Countermeasures. Final Report. DOT HS 806 563, April 1984. Washington, DC: National Highway Traffic Safety Administration.

Tests conspicuity-enhancing treatments in the field for pedestrians and bicyclists. 
Title: Blomberg, R.D., Hale, A., \& Preusser, D.F. Conspicuity for Pedestrians and Bicyclists: Definition of the Problem, Development and Test of Countermeasures. Final Report. DOT HS 806 563, April 1984

Problem Examined: Conspicuity refers to the state of being obvious to the eye or mind, having the capability of being easy to see. It is a necessary condition if pedestrian and bicyclist crashes are to be avoided. Conspicuity is thought of as mainly a nighttime prerequisite, but research has shown it to be a factor also in daytime crashes. In this study, experiments are conducted to examine how well commercial material and devices aid nighttime conspicuity.

Age Group(s) Addressed: All age groups

Crash Type(s) Involved: Two types selected for study (pedestrian: walking along roadway, and bicyclist: motorist overtaking/bicyclist not observed\}; other types also are relevant.

\begin{abstract}
A field experiment was conducted to determine the extent of conspicuity enhancement provided pedestrians and bicyclists at night by various commercially available retroreflective material and lights. The conspicuous material were designed to be worn or carried by the pedestrians and bicyclists. An operational highway environment with characteristics similar to the crash types being studied was used for data collection. Data were collected from alerted subjects $(\mathrm{n}=36)$ driving instrumented vehicles using low beam headlights. Field experimenters were used to model the conspicuity-enhancing material employing natural motion associated with walking and bicycling. The enhancement treatments for pedestrians included: dangle tags, flashlight, jogger's vest, retroreflective rings (wristbands, headbands, belts, ankle bands); bicyclist enhancements included: retroreflective strips and tubes on bicycle cranks and spokes, leg lamp, retroreflective fanny bumper and ankle bands.
\end{abstract}

Detection and recognition distances for the various experimental and baseline conditions were determined. The detection and recognition data collected for the experimental treatment were compared to respective baseline or untreated conditions and were interpreted in terms of various indices of "sufficient conspicuity."

Specific recommendations for use were:

- White clothing should not be used as a conspicuity enhancer. White alone is not sufficient to promote an acceptable level of safety. Safety campaigns should not promote the use of white clothing as a countermeasure but, rather should concentrate on retroreflective and active treatments for nighttime use and fluorescent material for daytime applications.

- Motorists should carry a flashlight or other active light source in their vehicles in case of a breakdown or crash. In addition, some retroreflective treatment should also be carried.

- Pedestrians who must undertake a purposeful nighttime trip should carry a flashlight or other light source and wear anthropometric-shaped retroreflective material like the Rings treatment. 
- If someone must bicycle at night, an active source, such as the Leg Lamp, supplemented by at least the standard CPSC reflectors should be used. A high-intensity bicycle lighting system would be useful for those who regularly ride at night. The best beacon type of flashing light would also appear to be a reasonable choice for both pedestrians and bicyclists.

- Joggers should wear a vest with two horizontal stripes of bright, retroreflective material in addition to carrying a flashlight or other active light source.

Related NHTSA Research: Blomberg, R.D., Cleven, A. M., and Edwards, J. M. Development of Safety Information Material and Media Plans for Elderly Pedestrians. Final Report, DOT HS 808 132, June 1993. Washington, DC: National Highway Traffic Safety Administration. Conspicuity problems and enhancement techniques are discussed.

Hale, A., \& Zeidler, P. Review of the Literature and Programs for Pedestrian and Bicyclist Conspicuity. Technical Report, DOT HS 806 564, April 1984. Washington, DC: National Highway Traffic Safety Administration.

Provides a good review of the conspicuity area.

Blomberg, R.D., \& Cleven, A.M. Development, Implementation, and Evaluation of a Pedestrian Safety Zone for Elderly Pedestrians. Final Report, DOT HS 808 692, February 1998. Washington, DC: National Highway Traffic Safety Administration.

Discusses some conspicuity-enhancing treatments for older pedestrians..

Blomberg, R.D., \& Cleven, A.M. Development, Implementation, and Evaluation of a Countermeasure Program for Alcohol-Involved Pedestrian Accidents. Final Report, DOT HS 809 067, July 2000. Washington, DC: National Highway Traffic Safety Administration. Employs a conspicuity-enhancement device (cap) for use by alcohol-involved pedestrians, along with other countermeasures. 


\section{COUNTERMEASURES}

\section{Integrated}

Title: Discretionary Agreement in Support of a Large City/Jurisdiction: Demonstration and Evaluation Program for Pedestrian Safety (DTNH22-98-H-0518). Project ongoing and final report in preparation.

Problem Examined: How do the previously tested countermeasures fare when integrated into an ongoing and committed local pedestrian and bicycle safety program? Are the techniques and the outcomes symbiotic?

\section{Age Group(s) Examined: All ages}

Crash Type(s) Involved: All pedestrian crash types

Abstract: The objective of this long-term demonstration project that began in 1998 is to determine the effectiveness of a combined pedestrian countermeasures program in Miami, Dade County, Florida and to determine the impacts of this program on reducing the traffic related injuries and associated costs within this area. Tasks included to carry out this effort were:

Task A - Analysis of the Pedestrian Safety Problem including identifying high crash zones and analyzing crashes within these zones.

Task B - Establishing local pedestrian safety partnership

Task C - Developing and implement program

Task D - Evaluating the program

Task E - Preparing reports and disseminate results

Related NHTSA Research: All of the countermeasures developed up to 1998 and described herein were made available to the Program as potential resources for application. 


\section{COUNTERMEASURES}

\section{Training}

Title: Berger, W. G. Urban Pedestrian Accident Countermeasures Experimental Evaluation: Volume II - Appendix A; Review of Education and Public Information Materials. Final Report, DOT HS 801 348, February 1975.

Problem Examined: What pedestrian safety activities are conducted by various jurisdictions State, city, and school district? The review attempts to reveal the characteristics of ongoing pedestrian safety programs and the extent to which these programs are responsive to the learning needs of the audience and the realities of the crash picture.

Age Group(s) Examined: All ages

Crash Type(s) Involved: All pedestrian crash types

Abstract: This technical appendix presents an overview of the national pedestrian safety effort. The appendix also reports the results of a survey of 48 ongoing educational programs being conducted in eight U.S. urban centers. A final chapter suggests procedures for the systematic development and evaluation of pedestrian safety programs.

Related NHTSA Research: Stutts, J.C., Hunter, W.W., Tracy, L., \& Wilkinson, W.C., III. Pedestrian and Bicyclist Safety: A Review of Key Program and Countermeasure Developments During the 1980s. Final Report, DOT HS 808 108, March 1992. Washington, DC: National Highway Traffic Safety Administration.

Provides a more recent update and overview of countermeasure programs.

Blatt, J., \& Dueker, R.L. Assessment of the Safety-Relevance of Pedestrian and Bicyclist Programs. Volume I: Conduct and Results. Final Report. DOT HS 806 436, April 1983. Washington, DC: National Highway Traffic Safety Administration.

Provides instrument for assessing the safety relevance of bicyclist safety education programs. 
Countermeasures - Training

Title: Blomberg, R.D., Leaf, W.A., Hale, A., Farrell, M.L., \& Cross, K.D. Identification and Development of Countermeasures for Bicyclist/Motor-Vehicle Problem Types Volume IMethod and Training Program Descriptions, Final Report, DOT HS 806 326, August 1982.

Problem Examined: Bicycle crash types were identified in 1977. This study defined three prototype countermeasures in the area of training for the crash problems.

Age Group(s) Addressed: All ages

Crash Type(s) Involved: All bicyclist types

Abstract: A detailed re-analysis of previously collected bicycle/motor-vehicle crash data (Cross and Fisher, 1977) was conducted to define potential countermeasures. Countermeasure development was then undertaken in the areas of Training (see Volume I), Public Education (see Volume II) and Model Regulations (see Volume III). Volume I: Three programs suitable for use as training material were produced. These were a fourth-grade comprehensive curriculum and separate, brief guides for parents and police officers. (Note: the fourth-grade curriculum was incorporated into another training program and is not available as a separate product.) Recommendations for implementing and field testing the developed training programs are included.

Related NHTSA Research: Blomberg, R.D., Leaf, W.A., Hale, A., Farrell, M.L., \& Cross, K.D. Identification and Development of Countermeasures for Bicyclist/Motor-Vehicle Problem Types Volume II - Public Information and Education Messages, Final Report, DOT HS-806-327, August 1982. Washington, DC: National Highway Traffic Safety Administration.

A companion volume developing PI\&E prototype messages for selected bicyclist problem types.

Blomberg, R.D., Leaf, W.A., Hale, A., Farrell, M.L., \& Cross, K.D. Identification and Development of Countermeasures for Bicyclist/Motor-Vehicle Problem Types Volume IIIModel Regulations, Final Report, DOT HS-806-328, August 1982. Washington, DC: National Highway Traffic Safety Administration.

A companion volume developing prototype model traffic safety regulations for selected bicyclist problem types.

Cross, K.D., \& Fisher, G. A study of Bicycle/Motor-Vehicle Accidents: Identification of Problem Types and Countermeasure Approaches. 3 Volumes. Final Report, DOT HS 803 315, DOT HS 803316 (Appendices), DOT HS 803317 (Coding Index), September 1977. Washington, DC: National Highway Traffic Safety Administration.

Provides the basic information on bicyclist/motor-vehicle crash types. 
Countermeasures - Training

Title: Cleven, A. M., and Blomberg, R.D. Development and Evaluation of a Pedestrian Safety Training Program for Elementary School Bus Riders. Final Report, DOT HS 808 267, December 1994.

Problem Examined: Children represent a significant proportion of pedestrians killed and injured in traffic crashes. While the actual school bus trip is among the safest forms of transportation, there are nevertheless substantial pedestrian risks associated with the total trip as the child walks to and from the bus stop, waits for the bus in a traffic environment, gets on and off the bus at home and at school, and sometimes crosses the street to and from the bus. The objective of this study was to develop and evaluate a comprehensive pedestrian safety program for the elementary school bus rider that is appropriate to all school environments - urban, suburban and rural.

Age Group(s) Addressed: Kindergarten through grade 6 children

Crash Type(s) Involved: Dart-out, midblock dash, backing vehicle, intersection dash, walking along the roadway, school-bus related, multiple threat

Abstract: The objective of this study was to develop and evaluate a comprehensive pedestrian safety program for elementary (kindergarten through grade 6) school bus riders. Existing material, crash data and State laws/regulations on school bus pedestrian safety were reviewed, and a list of 113 behaviors to be included in the program was developed. The major behavioral categories were: getting ready for school, walking to/from the bus stop, waiting at the bus stop, crossing to the bus, boarding the bus, riding the bus, exiting/crossing from the bus and evacuating the bus. The resultant program contains material for teachers, parents and bus drivers. They include separate Teacher's Guides for each of the seven grade levels. Incorporated in the program for children are two previously-produced NHTSA videos: Stop and Look with Willy Whistle and Walking with Your Eyes, and one newly-developed video titled Willy Whistle Rides the School Bus. A course poster completes the classroom material. Parent material include a video titled School Bus Safety Starts at Home and a brochure titled Reminder to Parents .. School Bus Safety Starts at Home. Bus driver material include a video titled When They're Not on the Bus and a brochure titled They're Pedestrians When They're Not on the Bus. Two promotional pieces (a flyer and an 8-page brochure) were prepared to assist NHTSA in marketing the program. All student material were evaluated in the East Ramapo Central School District, Spring Valley, New York, using a pre-post design with a comparison site. Statistically significant improvements were achieved in critical knowledge and skills as a result of student participation in the program.

The program was made available through the National Safety Council as the Walk-Ride-Walk: Getting to School Safely Program

Related NHTSA Research: Dueker, R. L., \& Chiplock, L.W. Identification and Feasibility Test of Specialized Rural Pedestrian Safety Training. Volume I: Program Development and Training. Final Report. DOT HS 806 256, March 1981. Washington, DC: National Highway Traffic Safety Administration.

Relates to earlier work on school bus safety training program in rural/suburban area. 
Chiplock, L.,W., \& Dueker, R.L. Identification and Feasibility Test of Specialized Rural Pedestrian Safety Training. Volume II: PedSafe Elementary Materials. Final Report, DOT HS 805 964, March 1981. Washington, DC: National Highway Traffic Safety Administration. Relates to earlier work on school bus safety training program in rural/suburban area.

Chiplock, L.W., Dueker, R.L., \& Bittner, S.R. Identification and Feasibility Test of Specialized Rural Pedestrian Safety Training. Volume IV: Pedsafe Audiovisual Scripts. Final Report, DOT HS 805 966, March 1981. Washington, DC: National Highway Traffic Safety Administration. Relates to earlier work on school bus safety training program in rural/suburban area. 


\section{COUNTERMEASURES}

\section{Messages}

Title: Blomberg, R.D., \& Preusser, D.F. Identification and Test of Pedestrian Safety Messages for Public Education Programs. Final Report. DOT HS 801 457, March 1975.

Problem Examined: To develop safety messages based on significant findings from pedestrian crash studies, and to ascertain through pre-testing if a behavioral change results from an understanding of the message content.

Age Group(s) Addressed: Children, adults, parents, drivers, ice cream vendor clients

Crash Type(s) Involved: Dart-out, pedestrian strikes vehicle, intersection dash, vehicle turn/merge, multiple threat, bus stop related, vendor - ice cream truck, freeway-expressway crossing.

Abstract: A review of the literature and data from pedestrian crash research studies was used as input to an analysis which developed 14 message contents. Each of these is directed at a specific aspect of the identified pedestrian crash problem. Seven of the messages were pretested to measure behavioral change. Of these, five produced significant positive shifts in behavior. It is concluded that public education can influence pedestrian behavior and is therefore a viable countermeasure to pedestrian crashes. Six of the message contents are recommended as ready for immediate field testing. Finally, recommendations for media campaigns for each of the developed message contents are provided.

Related NHTSA Research: Blomberg, R.D., Preusser, D.F., Hale, A., and Leaf, W.A. Experimental Field Test of Proposed Pedestrian Safety Messages: 3 Volumes: - This study carries the messages developed earlier through to a field evaluation. Volume I: Methods and Materials, Final Report, DOT HS 806-521, November 1983. Volume II: Child Messages, Final Report, DOT HS 806 522, November 1983. Volume III: Adult Messages, Final Report, DOT HS 806523 , November 1983. 
Title: Blomberg, R.D., Preusser, D.F., Hale, A., and Leaf, W.A. Experimental Field Test of Proposed Pedestrian Safety Messages: (3 Volumes) Volume I: Methods and Materials, Final Report, DOT HS 806 521, November 1983

Problem Addressed: How to use the pedestrian crash data gathered in the early 1970s to structure the content, presentation, and evaluation of public education messages designed to reduce specific types of pedestrian crashes.

Age Group(s) Addressed: Children and adults

Crash Type(s) Involved: Dart-out, vehicle turn/merge, multiple threat

Abstract: A detailed re-analysis of available pedestrian crash data was utilized to define three sets of pedestrian safety public information and education (PI\&E) messages. These messages were then produced and field tested. The objectives and theoretical background for the study are addressed in this Volume. The messages directed at child pedestrian crashes and using an animated character named "Willy Whistle" are covered in Volume II. Two sets of adult-oriented messages are the focus of Volume III. The success of these messages leads to the additional conclusion that PI\&E, in general, can be an effective countermeasure modality for modifying simple behaviors if adequate exposure is obtained.

Related NHTSA Research: Blomberg, R.D., \& Preusser, D.F. Identification and Test of Pedestrian Safety Messages for Public Education Programs. Final Report. DOT HS 801457 , March 1975. Washington, DC: National Highway Traffic Safety Administration.

Determined that safety messages can influence behavior.

Preusser, D.F., Blomberg, R.D., Edwards, J.M., Farrell, M. L., \& Preusser, C.W. The Development and Test of Urban and Rural Pedestrian Safety Messages. Final Report. DOT HS 806 682, January 1985 . Washington, DC: National Highway Traffic Safety Administration. Extended safety messages countermeasure approach to previously untouched crash types.

Preusser, D. F., \& Lund, A.K. And Keep on Looking: A Film to Reduce Pedestrian Crashes Among 9-12 Year Olds, Journal of Safety Research, Vol 19: 177-185, 1988. - Provides evidence of crash-reduction effects of child safety messages. 
Title: Preusser, D.F., Blomberg, R.D., Edwards, J.M., Farrell, M. L., \& Preusser, C.W. The Development and Test of Urban and Rural Pedestrian Safety Messages. Final Report. DOT HS 806 682, January 1985.

Problem Examined: It having been demonstrated that public education in the form of safety messages is effective in changing behavior and has potential for reducing crashes, this study sought to extend this experience to new target areas.

Age Group(s) Addressed: All age groups

Crash Type(s) Involved: Dart-out, midblock dash, intersection dash, pedestrian not in roadway, backing vehicle, disabled vehicle, school bus related, mail box, big wheel, child supervision, visual screen

Abstract: The objective of this project was to identify, develop and produce public education messages for pedestrian safety. Pedestrian crash types and situations which had not been previously addressed through public education were selected. These included "Riding Toys," "Backing," "Pedestrian Not in Road", "Visual Screens", "Intersection Dash," "Darts and Dashes," "School Bus," "Child Supervision," "Elderly," "Mail Box," and "Disabled Vehicle". Each type was analyzed to determine specific behavioral advice that could be adopted by pedestrians, parents or drivers and could be expected to reduce crashes. Prototype TV and radio scripts, pamphlets and posters were developed to carry this advice to identified target groups. These prototype media forms underwent focus group reaction testing. Three TV spots and a 15 minute in-class film were produced to finished form. The spots were targeted to adult pedestrians (Intersection Dash, :30 seconds), child pedestrians (Intersection Dash, :60 seconds) and children who play on riding toys ( 60 seconds). The in-class film was designed to follow the original Willy Whistle film and present more complex traffic situations to older children (7-14 years).

Related NHTSA Research: Blomberg, R.D., \& Preusser, D.F. Identification and Test of Pedestrian Safety Messages for Public Education Programs. Final Report. DOT HS 801 457, March 1975. Washington, DC: National Highway Traffic Safety Administration. Demonstrated that messages can change behavior.

Blomberg, R.D., Preusser, D.F., Hale, A., and Leaf, W.A. Experimental Field Test of Proposed Pedestrian Safety Messages: 3 Volumes: - Provides evidence of crash reductions from use of safety messages. Volume I: Methods and Materials, Final Report, DOT HS 806-521, November 1983. Volume II: Child Messages, Final Report, DOT HS 806 522, November 1983.

Volume III: Adult Messages, Final Report, DOT HS 806 523, November 1983.

Preusser, D. F., \& Lund, A.K. And Keep on Looking: A Film to Reduce Pedestrian Crashes Among 9-12 year Olds, Journal of Safety Research, Vol 19: 177-185, 1988 - Provides evidence of crash-reduction effects of safety messages.

Snyder, M.B., \& Knoblauch, R.L. Pedestrian Safety: The Identification of Precipitating Factors and Possible Countermeasures. Final Report, Volumes I, II (Appendices\}, DOT HS-800-403 
and DOT HS 800 404, January 1971. Washington, DC: National Highway Traffic Safety Administration.

First study to identify the crash types now targeted in the messages studies. 
Title: Blomberg, R.D., Leaf, W.A., Hale, A., Farrell, M.L., \& Cross, K.D. Identification and Development of Countermeasures for Bicyclist/Motor-Vehicle Problem Types Volume II - Public Information and Education Messages, Final Report, DOT HS 806 327, August 1982.

Problem Examined: Bicycle crash types were identified in 1977. This study defined 15 prototype countermeasures in the area of public information and education for the crash problems.

Age Group(s) Addressed: Bicyclists, motorists

Crash Type(s) Involved: Nearly all bicyclist crash types

Abstract: A detailed re-analysis of previously collected bicycle/motor-vehicle crash data (Cross and Fisher, 1977) was conducted to define potential countermeasures. Countermeasure development was then undertaken in the areas of Training (see Volume I), Public Education (see Volume II) and Model Regulations (see Volume III). Regarding Volume II, a set of ten TV spots in story board form, four radio scripts, and a camera-ready reproducible of a poster were developed. This volume is devoted to a discussion of the public information and education messages developed. For the purpose of this effort and most other NHTSA work in bicycle and pedestrian safety, a message has been defined as an educational countermeasure suitable for distribution through the mass media. Alternately, a "message" might be thought of as information which is distributed in a manner such that the distributing agency does not have direct control over the specific individuals who receive the material. Recommendations for implementing and field testing the developed messages are included.

Related NHTSA Research: Blomberg, R.D., Leaf, W.A., Hale, A., Farrell, M.L., \& Cross, K.D. Identification and Development of Countermeasures for Bicyclist/Motor-Vehicle Problem Types Volume I-Method and Training Program Descriptions, Final Report, DOT HS 806 326, August 1982.

Blomberg, R.D., Leaf, W.A., Hale, A., Farrell, M.L., \& Cross, K.D. Identification and Development of Countermeasures for Bicyclist/Motor-Vehicle Problem Types Volume IIIModel Regulations, Final Report, DOT HS 806 328, August 1982.

Cross, K.D., \& Fisher, G. A study of Bicycle/Motor-Vehicle Accidents: Identification of Problem Types and Countermeasure Approaches. 3 Volumes. Final Report, DOT HS 803 315, DOT HS 803316 (Appendices), DOT HS 803317 (Coding Index) September 1977. 
Title: Blomberg, R.D., Preusser, D.F., Hale, A., and Leaf, W.A. Experimental Field Test of Proposed Pedestrian Safety Messages: (3 Volumes) Volume II: Child Messages, Final Report, DOT HS 806 522, November 1983

Problem Addressed: How to use the pedestrian crash data gathered in the early 1970s to structure the content, presentation, and evaluation of public education messages designed to reduce specific types of pedestrian crashes.

Age Group(s) Addressed: Children

Crash Type(s) Involved: Dart-out, vehicle turn/merge, multiple threat

Abstract: A detailed re-analysis of available pedestrian crash data was utilized to define three sets of pedestrian safety public information and education (PI\&E) messages. These messages were then produced and field tested. The objectives and theoretical background for the study are addressed in Volume I. The messages directed at child pedestrian crashes and using an animated character named "Willy Whistle" are covered in this Volume. The child messages were successful in reducing pedestrian crashes in three test cities. It was concluded that these messages are viable pedestrian crash countermeasures. The success of these messages leads to the additional conclusion that PI\&E, in general, can be an effective countermeasure modality for modifying simple behaviors if adequate exposure is obtained.

Related NHTSA Research: Blomberg, R.D., \& Preusser, D.F. Identification and Test of Pedestrian Safety Messages for Public Education Programs. Final Report. DOT HS 801457 , March 1975. Washington, DC: National Highway Traffic Safety Administration.

Determined that safety messages can influence behavior.

Preusser, D.F., Blomberg, R.D., Edwards, J.M., Farrell, M. L., \& Preusser, C.W. The Development and Test of Urban and Rural Pedestrian Safety Messages. Final Report. DOT HS 806 682, January 1985. Washington, DC: National Highway Traffic Safety Administration. Extended safety messages countermeasure approach to previously untouched crash types.

Preusser, D. F., \& Lund, A.K. And Keep on Looking: A Film to Reduce Pedestrian Crashes Among 9-12 year Olds, Journal of Safety Research, Vol 19: 177-185, 1988 - Provides evidence of crash-reduction effects of child safety messages. 
Title: Preusser, D. F., \& Lund, A.K. And Keep on Looking: A Film to Reduce Pedestrian Crashes Among 9-12 Year Olds, Journal of Safety Research, Vol 19: 177-185, 1988.

Problem Addressed: Knowledge of child safety messages and assessment of crash reduction.

Age Group(s) Addressed: Children 9 to 12 years old

Crash Type(s) Involved: Intersection darts and dashes, turning vehicles, backing vehicles, parking lot crashes

\begin{abstract}
This study involved a field evaluation of NHTSA's pedestrian safety film called And Keep on Looking that was aimed at children aged 9 to 12. There was an increase in safe street crossing knowledge among Connecticut children who viewed the film and some improvement in safe street crossing behavior among Seattle children who viewed the film. Crash reduction was assessed in a two-year citywide field test conducted in Milwaukee. That test indicated a crash reduction of more than $20 \%$ for Milwaukee children in the 9 to 12 year age group compared with children in areas surrounding Milwaukee and children in comparison cities.
\end{abstract}

Related NHTSA Research: Blomberg, R.D., \& Preusser, D.F. Identification and Test of Pedestrian Safety Messages for Public Education Programs. Final Report. DOT HS 801457 , March 1975. Washington, DC: National Highway Traffic Safety Administration.

Determined that safety messages can influence behavior.

Preusser, D.F., Blomberg, R.D., Edwards, J.M., Farrell, M. L., \& Preusser, C.W. The Development and Test of Urban and Rural Pedestrian Safety Messages. Final Report. DOT HS 806 682, January 1985 . Washington, DC: National Highway Traffic Safety Administration. Extended safety messages countermeasure approach to previously untouched crash types.

Blomberg, R.D., Preusser, D.F., Hale, A., and Leaf, W.A. Experimental Field Test of Proposed Pedestrian Safety Messages: (3 Volumes) Volume II: Child Messages, Final Report, DOT HS 806 522, November 1983. Washington, DC: National Highway Traffic Safety Administration. Concluded that the messages were viable pedestrian crash countermeasures. 
Title: Blomberg, R.D., Preusser, D.F., Hale, A., \& Leaf, W.A. Experimental Field Test of Proposed Pedestrian Safety Messages: (3 Volumes) Volume III: Adult Messages, Final Report, DOT HS 806 523, November 1983. Washington, DC: National Highway Traffic Safety Administration.

Problem Addressed: How to use the pedestrian crash data gathered in the early 1970s to structure the content, presentation, and evaluation of public education messages designed to reduce specific types of pedestrian crashes.

\title{
Age Group(s) Addressed: Adults
}

Crash Type(s) Involved: Vehicle turn/merge, multiple threat

\begin{abstract}
A detailed re-analysis of available pedestrian crash data was utilized to define three sets of pedestrian safety public information and education (PI\&E) messages. These messages were then produced and field-tested. The objectives and theoretical background for the study are addressed in Volume I. Two sets of adult-oriented messages are the focus of this volume. The adult messages yielded some positive results, especially for Spanish-speaking adult pedestrians. It was concluded that these messages are viable pedestrian crash countermeasures. The success of these messages leads to the additional conclusion that PI\&E, in general, can be an effective countermeasure modality for modifying simple behaviors if adequate exposure is obtained.
\end{abstract}

Related NHTSA Research: Blomberg, R.D., \& Preusser, D.F. Identification and Test of Pedestrian Safety Messages for Public Education Programs. Final Report. DOT HS 801457 , March 1975. Washington, DC: National Highway Traffic Safety Administration.

Determined that safety messages can influence behavior.

Preusser, D.F., Blomberg, R.D., Edwards, J.M., Farrell, M. L., \& Preusser, C.W. The Development and Test of Urban and Rural Pedestrian Safety Messages. Final Report. DOT HS 806 682, January 1985. Washington, DC: National Highway Traffic Safety Administration. Extended safety messages countermeasure approach to previously untouched crash types.

Preusser, D. F., \& Lund, A.K. And Keep on Looking: A Film to Reduce Pedestrian Crashes Among 9-12 Year Olds, Journal of Safety Research, Vol 19: 177-185, 1988.

Provides evidence of crash-reduction effects of child safety messages. 


\title{
COUNTERMEASURES
}

\section{Regulations}

Title: Blomberg, R.D., Hale, A., \& Kearney, E.F. Development of Model Regulations for Pedestrian Safety. Final Report. DOT HS 801 287, November 1974. Washington, DC: National Highway Traffic Safety Administration.

Problem Examined: The purpose of this study was to develop a set of model rules, regulations, codes, ordinances and related procedures in eight specific subject areas which, if adopted by States and local jurisdictions, would reduce pedestrian crashes.

Age Group(s) Addressed: All age groups

Crash Type(s) Involved: Ice cream vendor, non-pedestrian activity in roadway (road workers), dismounted motorist, bus-stop-related, intersection dash, multiple threat, dart-out, backing vehicle

\begin{abstract}
Nine model regulations to improve pedestrian safety are presented. Each is targeted at one or more specific types of pedestrian crashes identified in previous research and is intended for codification with existing State or municipal vehicle and traffic codes. The regulations were developed and subjected to public/official acceptance testing through a mailed survey. The final regulations obtained in the report are based on detailed analysis tempered by the survey results. In addition, implementation considerations for each regulation are discussed. The nine regulations cover: ice cream vending; road work sites; freeway vehicle stops; bus stop location; parking near intersections and crosswalks; vehicle overtaking; on-street parking in new or redeveloped residential areas; pedestrian crash information and countermeasures; and backing signals.
\end{abstract}

Related NHTSA Research: DeBartolo, K.B., Preusser, D.F., \& Blomberg, R.D. Enforcement Frequency, Sanctions and Compliance Level for Pedestrian Safety. Final Report. DOT HS 803 650, April 1978. Washington, DC: National Highway Traffic Safety Administration. Showed that increased enforcement can lead to improved motorist compliance with traffic regulation.

Hale, A., Blomberg, R.D., \& Preusser, D.F. Experimental Field Test of the Model Ice Cream Truck Ordinance In Detroit. Final Report, DOT HS 803 410, April 1978. Washington, DC: National Highway Traffic Safety Administration.

NHTSA's most effective crash-reducing model regulation.

Hale, A., Blomberg, R.D., \& Kearney, E.F. Model Regulations and Public Education for RuralSuburban Pedestrian Safety, Final Report, DOT HS 805 639, August 1980. Washington, DC: National Highway Traffic Safety Administration.

Looks at the rural/suburban area through the countermeasure mechanism of traffic safety regulations. 
Ulmer, R.G., Leaf, W.A., and Blomberg, R.D. Analysis of the Dismounted Motorist and RoadWorker Model Pedestrian Safety Regulations. Final Report, DOT HS 806 445, August 1982. Washington, DC: National Highway Traffic Safety Administration.

Describes field test of a proposed model regulation. 
Title: Hale, A., Blomberg, R.D., \& Kearney, E.F. Model Regulations and Public Education for Rural-Suburban Pedestrian Safety, Final Report, DOT HS 805 639, August 1980. Washington, DC: National Highway Traffic Safety Administration.

Problem Examined: Traffic regulations are a potentially viable means of controlling unsafe behaviors implicated in pedestrian crashes. This study looks at the area of rural and suburban pedestrian crashes through the countermeasure mechanism of traffic regulations.

Age Group(s) Addressed: All ages

Crash Type(s) Involved: Walking along the roadway, disabled vehicle related, working on the roadway, school-bus-related, mailbox-related, interchange dash/dart-out

Abstract: The objectives of this study were to review the rural-suburban pedestrian crash data (Knoblauch, 1977) and freeway pedestrian crash data (Knoblauch, Moore, \& Schmitz, 1976) and determine which crash types were amenable to countermeasures development. Countermeasure classes considered were model traffic regulations and public information and education (PI\&E). The results of the analysis indicated that the development of four prototype regulations to serve as legislative models appeared to be promising in reducing the target crash types. The four model regulations include the:

- Model Regulation for School Bus Pedestrians;

- Model Regulation for Pedestrians on Highways;

- Model Freeway Restrictions Regulation; and

- Model Vehicle Hazard Warning Lights Regulations.

Four media packages were also seen as potentially effective countermeasures for their target crash types. Initial concepts are presented for the following media packages:

- School Bus Driver Pamphlet;

- Dismounted Motorist Public Service Announcements;

- Mailbox Safety Flyer; and

- Road Worker Pamphlet.

A complete discussion of the background crash data, the countermeasure objectives, content rationale, and the requirements for further development, implementation and testing (where appropriate) is provided for the model regulations and media packages.

Related NHTSA Research: Blomberg, R.D., Hale, A., \& Kearney, E.F., Development of Model Regulations for Pedestrian Safety. Final Report, DOT HS 801 287, November 1974.

Washington, DC: National Highway Traffic Safety Administration.

Proposed model regulations for urban areas. 
Countermeasures - Regulations

Title: Leaf, W.A., \& Blomberg, R.D. Development and Test of Selected Model Pedestrian Safety Regulations. DOT HS 805 901, April 1981. Washington, DC: National Highway Traffic Safety Administration.

Problem Examined: Model traffic safety regulations designed to prevent selected types of pedestrian crashes are tested in the field. A new model regulation is drafted.

Age Group(s) Addressed: All age groups

Crash Types Involved: Dart-out, multiple threat

\begin{abstract}
Two model regulations to remove parking - one from suburban streets in daylight hours and one on the last 50 feet of the approach to crosswalks - were designed in previous work to prevent pedestrian "dart and dash" crashes by removing screening vehicles, thereby allowing pedestrians and motorists to more easily see each other and react as needed. To evaluate the safety benefits of the regulations, a study was conducted in New York City. The residential areas of Manhattan have one-side-only parking for three hours per day according to a scheme balanced by time of day and affected side of street. Crash data from 1974-1977 were screened and reports were reviewed. Based on 835 pedestrian crashes, no changes in crash distributions were found when the alternate-side parking regulation was in effect. Supplementary observations in the test areas showed good but imperfect compliance with the parking bans and virtually no changes in pedestrian frequency of appearance and cross behaviors as a function of parking changes. The pattern of results plus discrepancies between the New York study situation and the model regulations meant that little could be concluded with respect to the effectiveness or non-effectiveness of the regulations. Guidelines for further research were presented, stressing the need for direct comparability to the model regulations.
\end{abstract}

In a related activity, support material were prepared for a third model regulation, one requiring motorists to stop prior to proceeding past another vehicle stopped before a crosswalk. The regulation is designed to protect against "multiple threat" type pedestrian crashes. Although its effectiveness was not examined in this study, this regulation promises to reduce crashes between the overtaking vehicle and pedestrians passing the stopped vehicle. Material to help pass and publicize the regulation was drafted for two western States with a confirmed crash problem of this type. Arizona showed interest in possibly implementing the model law, with contingent evaluation assistance from NHTSA. Suggestions are offered for a follow-up schedule for the State and for NHTSA.

Related NHTSA Research: Blomberg, R.D., Hale, A., \& Kearney, E.F. Development of Model Regulations for Pedestrian Safety. Final Report to the National Highway Traffic Safety Administration, U.S. Department of Transportation, DOT HS 801 287, November 1974. Washington, DC: National Highway Traffic Safety Administration. Presents nine model regulations to improve pedestrian safety. 
Title: Ulmer, R.G., Leaf, W.A., \& Blomberg, R.D. Analysis of the Dismounted Motorist and Road-Worker Model Pedestrian Safety Regulations. Final Report. DOT HS 806 445, August 1982. Washington, DC: National Highway Traffic Safety Administration.

Problem Examined: This study takes two model pedestrian safety regulations previously developed by NHTSA and examines their potential safety benefits in an experimental field situation.

Age Group(s) Addressed: Adults

Crash Type(s) Involved: Dismounted motorist, road worker

Abstract: Two pedestrian model regulations previously developed by NHTSA were studied to determine their potential safety benefits. One regulation was concerned with the disabledvehicle situation and called on motorists to position themselves and their vehicles as safely as possible and to employ conspicuity-enhancing devices and materials. This regulation was studied in an experimental field setting in which the operational features of the regulation were examined. The results of the study showed that deploying fusees or warning triangles in conjunction with four-way flashers significantly reduced the speed and shifted the placement of vehicles passing a simulated disabled vehicle during daytime and nighttime conditions. No substantial evidence was found to indicate that wearing fluorescent and retroreflective materials influenced the course or speed of passing motorists. It is recommended that the portions of the model regulation concerned with the positioning of vehicles and deployment of hazard warning devices be made available to locales seeking countermeasures against this crash type. Provisions related to wearing conspicuous materials should be deleted as mandatory requirements.

The second model regulation studied involved persons performing road work and called for workers to wear approved fluorescent and retroreflective materials, for standard traffic control devices to be employed, for permits and inspection of road work sites and for drivers to yield to workers and workers to avoid sudden movements into the path of vehicles. Detailed analyses of crash reports for cases where road workers were struck, indicated that there were a variety of precipitating factors involved and that rather than being a unitary crash type, these crashes were made up of several sub-types. It was concluded that even if the portions of the model regulation related to worker conspicuity and dart-out behavior were fully effective, only a minority of road worker crashes would be affected. 
Title: Blomberg, R. D., Leaf, W. A., Hale, A., Farrell, M. L., \& Cross, K. D. Identification and Development of Countermeasures for Bicyclist/Motor-Vehicle Problem Types Volume III Model Regulations, Final Report, DOT HS 806 328, August 1982. Washington, DC: National Highway Traffic Safety Administration.

Problem Examined: Bicycle crash types were identified in 1977. This study defined eight prototype countermeasures in the area of model traffic safety regulations for the crash problems.

Age Group(s) Addressed: Bicyclists, motorists, property owners, police

Crash Type(s) Involved: Nearly all bicyclist crash types

Abstract: A detailed re-analysis of previously collected bicycle/motor-vehicle crash data (Cross and Fisher, 1977) was conducted] to define potential countermeasures. Countermeasure development was then undertaken in the areas of Training (see Volume I), Public Education (see Volume II) and Model Regulations (see Volume III). As regards Volume III, eight model State laws or municipal ordinances were developed and are reported therein. These are: Model Bicyclist Conspicuity Law, Model Law for Bicyclist Position on the Highway, Model Highway Entry Law, Model Minimum Age Law for Bicyclists, Model Driveway Parking Ordinance, Model Law to Remove Visual Obstructions, Model Regulation to Prohibit Riding Bicycles on Sidewalks, Model Bicycle Safety Patrol and Violation Disposition Ordinance.

Recommendations for implementing and field testing the developed regulations are included.

Related NHTSA Research: Blomberg, R.D., Leaf, W.A., Hale, A., Farrell, M.L., \& Cross, K.D. Identification and Development of Countermeasures for Bicyclist/Motor-Vehicle Problem Types Volume I-Method and Training Program Descriptions, Final Report, DOT HS 806 326, August 1982. Washington, DC: National Highway Traffic Safety Administration.

Develops three prototype training programs for selected bicyclist problem types.

Blomberg, R.D., Leaf, W.A., Hale, A., Farrell, M.L., \& Cross, K.D. Identification and

Development of Countermeasures for Bicyclist/Motor-Vehicle Problem Types Volume II - Public Information and Education Messages, Final Report, DOT HS 806 327, August 1982.

Washington, DC: National Highway Traffic Safety Administration.

Develops 15 prototype PI\&E messages for selected bicyclist problem types.

Cross, K.D., \& Fisher, G. A Study of Bicycle/Motor-Vehicle Accidents: Identification of Problem Types and Countermeasure Approaches. 3 Volumes. Final Report, DOT HS 803 315, DOT HS 803316 (Appendices), DOT HS 803 317, September 1977. Washington, DC: National Highway Traffic Safety Administration.

Provides basic information on bicyclist problem types. 
Title: Hale A., Shapiro, R.G., Blomberg, R.D., \& Kearney, E.F. Development and Test of Rural Pedestrian Safety Countermeasures. Final Report. DOT HS 806 518, December 1983.

Washington, DC: National Highway Traffic Safety Administration.

Problem Examined: Taking the next step in the process of developing model pedestrian safety regulations, this study undertook the task of testing a regulation for its effectiveness in changing behavior.

Age Group(s) Addressed: School bus riders

Crash Type(s) Involved: School-bus-related

Abstract: Prior to any promulgation by NHTSA of four model traffic regulations for rural pedestrian safety it was the objective of this study to assess, where feasible, the potential effectiveness of these regulations to prevent pedestrian crashes. The model regulations/ legislative packages developed under a previous contract (DOT-HS-7-01753) were titled:

- Model Regulation for School Bus Pedestrians;

- Model Regulation for Pedestrians on Highways;

- Model Freeway Walking Restrictions Regulation; and

- Model Vehicle Hazard Warning Lights Regulation.

After extensive analysis only the Model Regulation for School Bus Pedestrians was deemed feasible for testing and further development. The results of studies conducted on school bus driver experiences and school bus passing violations to assess the potential effectiveness of various aspects of this regulation are described. A revised model school bus regulation, incorporating the results of the tests conducted, along with concepts for public information and education to support statutory enactment and compliance with the model are presented. While the revised Model Regulation for School Bus Pedestrians is the principal product of this study, many of its provisions may be implemented without the need for a regulatory format, e.g., the functional requirements for a system to observe pedestrians near the school bus.

Related NHTSA Research: DeBartolo, K.B., Preusser, D.F., \& Blomberg, R.D. Enforcement Frequency, Sanctions and Compliance Level for Pedestrian Safety. Final Report. DOT HS 803 650, April 1978. Washington, DC: National Highway Traffic Safety Administration.

Demonstrated a relationship between increased enforcement and motorist compliance to a pedestrian-relevant traffic regulation.

Hale, A., Blomberg, R.D., \& Preusser, D.F. Experimental Field Test of the Model Ice Cream Truck Ordinance In Detroit. Final Report, DOT HS 803 410, April 1978. Washington, DC: National Highway Traffic Safety Administration.

Demonstrated the crash-reduction capability of a model regulation.

Ulmer, R.G., Leaf, W.A., \& Blomberg, R.D. Analysis of the Dismounted Motorist and RoadWorker Model Pedestrian Safety Regulations. Final Report. DOT HS 806 445, August 1982. Washington, DC: National Highway Traffic Safety Administration. 
Demonstrated the effectiveness of parts of a model traffic regulation. 


\section{COUNTERMEASURES}

\section{Bicycle Helmet Promotions}

Title: Tracy, L. Procedures and Resource Guide for Bicycle Helmet Promotions: A Review of Bicycle Helmet Promotions in the United States. Final Report, DOT HS 807 963, September 1992. Washington, DC: National Highway Traffic Safety Administration.

Problem Examined: Who is promoting bicycle helmet use in the United States and what program elements are common to successful promotions?

Age Group(s) Addressed: All ages

\section{Crash Type(s) Involved: Not applicable}

Abstract: Review of 231 national, State, and local bicycle helmet promotions in the United States based on a Bicycle Federation of America survey conducted in the summer of 1991. Report identifies trends, common elements of successful programs, goals, strategies, and level and type of evaluation. Examples of helmet promotion resources, material, guides, and manuals are discussed. Various factors that initiated promotions and barriers that challenged progress are also included. Directory of programs lists contact information plus target audience, promotion strategy, level of funding, project length, geographic area, level of evaluation, and whether or not the promotion was a coalition effort. 


\title{
COUNTERMEASURES
}

\section{Enforcement}

Title: Singer, S. Pedestrian Regulation Enforcement and the Incidence of Pedestrian Accidents. Final Report, National Highway Safety Bureau, SSD-69-726, August 1969.

Problem Examined: Determine the composition, enforcement, and effectiveness of pedestrian protection ordinances.

Age Group(s) Addressed: All ages

\section{Crash Type(s) Involved: Not applicable}

\begin{abstract}
Pedestrian deaths account for approximately $20 \%$ of the total fatalities resulting from motor vehicle crashes. Approximately two-thirds of all pedestrian casualties occur while crossing or entering streets. In response to the pedestrian crash problem municipal authorities have devised a variety of pedestrian safety programs, including the adoption of pedestrian protection ordinances and an attempt to increase compliance with these laws by the threat, actual or implied, of legal sanctions. However, no objective research studies have been performed to adequately evaluate the effectiveness of these programs, or to develop practical police countermeasures to prevent pedestrian casualties.
\end{abstract}

This project is one of a series of research efforts regarding various facets of the pedestrian crash problem being sponsored by the National Highway Safety Bureau. The objective of this study is an investigation of pedestrian protection ordinances, their enforcement and their effectiveness in crash reduction in urban areas. The research program described in this report was structured around three basic tasks - a review of the technical traffic safety literature, including an examination of pedestrian protection ordinances; the collection and analysis of existing statistical data pertaining to the enforcement of pedestrian ordinances and their relationship to the incidence of pedestrian crashes; and the conduct of a field experiment designed to investigate the effect of an increase in the level of enforcement on pedestrian violation behavior in crossing urban intersections. 
Title: DeBartolo, K.B., Preusser, D.F., \& Blomberg, R.D. Enforcement Frequency, Sanctions and Compliance Level for Pedestrian Safety. Final Report. DOT HS 803 650, April 1978. Washington, DC: National Highway Traffic Safety Administration.

Problem Examined: What is the effect of enforcement on motorist compliance with parking bans having a potential pedestrian-safety benefit?

Age Group(s) Addressed: Motorists and children

Crash Type(s) Involved: Dart-out, midblock dash

\begin{abstract}
Parked vehicles can create a visual screen such that oncoming motorists and crossing pedestrians cannot see each other. One proposed safety countermeasure is parking bans for specific times at high-risk locations. The purpose of this study was to investigate the effect of enforcement on motorist compliance with such time-phased parking bans. The study used the time-phased alternate side parking regulations in New York City. Enforcement varied from no increase above normally occurring enforcement to two additional enforcement visits per day. The results showed that increased enforcement can lead to improved motorist compliance. However, the timing of the additional visits within the period of prohibited parking is critical. The observed effects developed slowly and extinguished slowly. Recommendations are offered for employing and enforcing time-phased parking bans.
\end{abstract}

Related NHTSA Research: Blomberg, R.D., Hale, A., \& Kearney, E.F. Development of Model Regulations for Pedestrian Safety. Final Report. DOT HS 801 287, November 1974. Washington, DC: National Highway Traffic Safety Administration. Contains model parking regulations. 


\section{COUNTERMEASURES}

\section{Engineering}

Title: Berger, W. G. Urban Pedestrian Accident Countermeasures Experimental Evaluation: Volume I - Behavioral Evaluation Study. Final Report, DOT HS 801 346, February 1975. Washington, DC: National Highway Traffic Safety Administration.

Problem Examined: Study examines whether specific pedestrian safety countermeasures are effective in changing pedestrian and driver behaviors.

Age Group(s) Addressed: All ages

Crash Type(s) Involved: Dart-out, intersection dash, vehicle turn/merge, pedestrian strikes vehicle, multiple threat, bus-stop-related, vendor - ice cream truck

Abstract: A series of site and crash specific pedestrian safety countermeasures had been developed in a previous study, but the effectiveness of these countermeasures had not been empirically evaluated. This project focused on the determination of the effectiveness of nine safety countermeasures. These included: preventive markings ("CAUTION" painted on pavement), median barriers, crosswalk setbacks, midblock crosswalk, diagonal parking, meter post barriers, stop line relocation, ice cream vendor warning lights, and bus stop relocations. A series of behavioral studies was conducted to determine the extent to which the proposed countermeasures inhibit undesirable vehicular and pedestrian behaviors. These studies, conducted in eight cities, evaluated the behavioral effects associated with the installation of a countermeasure by means of pairing each experimental site with a control site in a pre-post design. Data collection methods included mechanical recording of vehicle speed and headway, and time-lapse photography, and manual coding of pedestrian and vehicle behavior. During the 204 days of data collection, the crossing behaviors of over 16,000 pedestrians were characterized. The crash reduction potential of the various countermeasures was assessed. The design and implementation problems associated with the countermeasures were also discussed. 


\section{ALCOHOL}

Title: Zylman, R., Blomberg, R.D., \& Preusser, D. F. A Review of the Literature on the Involvement of Alcohol in Pedestrian Collisions Resulting in Death and Injury. Interim Report, DOT HS 801 413, February 1975. Washington, DC: National Highway Traffic Safety Administration.

Problem Examined: What role does alcohol play in the magnitude and nature of pedestrian crashes?

Age Group(s) Addressed: All ages

Crash Type(s) Involved: All pedestrian crash types

Abstract: A review of the literature on the existing state of knowledge of the role of alcohol in pedestrian crashes indicates that little is currently known. It is concluded that more data is needed before the extent of any pedestrian safety problems involving alcohol can be quantified or countermeasures can be devised. Virtually nothing is known about this serious problem. However, the bits of information available and the similarity of the pedestrian-alcohol situation to the more extensively research area of drinking-driving permits an estimate of what is not known and how this needed knowledge can be acquired.

Related NHTSA Research: Blomberg, R.D., Preusser, D.F., Hale, A., \& Ulmer, R.G. A Comparison of Alcohol Involvement in Pedestrians and Pedestrian Casualties. Final Report, DOT HS 805 521, October 1979. Washington, DC: National Highway Traffic Safety Administration.

Landmark study identifying and quantifying alcohol-pedestrian problem.

Blomberg, R.D., \& Cleven, A.M. Development, Implementation, and Evaluation of a Countermeasure Program for Alcohol-Involved Pedestrian Crashes. Final Report, DOT HS 809 067, July 2000. Washington, DC: National Highway Traffic Safety Administration.

Project field tested techniques for reducing the alcohol-involved pedestrian crash problem.

Leaf, W.A., \& Preusser, D.F. Identification of Alcohol-Pedestrian Crash Problems Among Selected Racial/Ethnic Groups. Final Report, DOT HS 808 641, September 1997. Washington, DC: National Highway Traffic Safety Administration. Further definition of the pedestrian alcohol problem. 
Title: Blomberg, R.D., Preusser, D.F., Hale, A., \& Ulmer, R.G. A Comparison of Alcohol Involvement in Pedestrians and Pedestrian Casualties. Final Report, DOT HS 805 249, October 1979. Washington, DC: National Highway Traffic Safety Administration.

Problem Examined: To determine the incidence of alcohol in adult pedestrian victims, and whether alcohol is overrepresented in such crashes when compared to non-crash controls.

Age Group(s) Addressed: Adults (14+ years)

Crash Type(s) Involved: Nearly all pedestrian crash types

Abstract: The objectives of this study were to determine the frequency of alcohol involvement in adult $\left(14\right.$ years $\left.{ }^{+}\right)$pedestrian fatalities and injuries; determine if alcohol was overrepresented; determine the causal role of alcohol; and suggest countermeasures. Pedestrian fatalities were sampled through the New Orleans coroner; non-fatal pedestrian victims were sampled through a large New Orleans hospital; and crash and control data were gathered via follow-up interviews, roadside interviews and police files. Results showed that $50 \%$ of the pedestrian fatal and nonfatal victims had been drinking. Blood alcohol concentrations were extremely high. Approximately $50 \%$ of those who had been drinking had BACs of $.20 \mathrm{~g} / \mathrm{dL}$ or higher. Victims were compared to three distinct control groups. The most conservative group (age and sex, matched at the crash site) showed relative risk of a crash increasing dramatically at BACs of .20 $\mathrm{g} / \mathrm{dL}$ or more. The least conservative (random group) showed relative risk increasing dramatically at BACs of $.10 \mathrm{~g} / \mathrm{dL}$ or more. Alcohol-involved pedestrians were more often middle-aged males, struck at night, on weekends, and exhibited a variety of social and personal problems. "Dart and Dash," "Pedestrian Strikes Vehicle" and "Not Classifiable" crash types were common. Legal, educational, engineering, and rehabilitation countermeasure approaches are discussed.

Related NHTSA Research: Leaf, W.A., \& Preusser, D.F. Identification of Alcohol-Pedestrian Crash Problems Among Selected Racial/Ethnic Groups. Final Report, DOT HS 808 641, September 1997. Washington, DC: National Highway Traffic Safety Administration. Further definition of the pedestrian alcohol problem.

Blomberg, R.D., \& Cleven, A.M. Development, Implementation, and Evaluation of a Countermeasure Program for Alcohol-Involved Pedestrian Crashes. Final Report, DOT HS 809 067, July 2000.Washington, DC: National Highway Traffic Safety Administration. Project field tested techniques for reducing the alcohol-involved pedestrian crash problem.

Zylman, R., Blomberg, R.D., \& Preusser, D. F. A Review of the Literature on the Involvement of Alcohol in Pedestrian Collisions Resulting in Death and Injury. Interim Report, DOT HS 801 413, February 1975. Washington, DC: National Highway Traffic Safety Administration. Early literature review project which outlined the existing knowledge about the alcoholpedestrian problem. 
Title: Blomberg, R.D., \& Cleven, A.M. Development, Implementation, and Evaluation of a Countermeasure Program for Alcohol-Involved Pedestrian Crashes. Final Report, DOT HS 809 067, July 2000. Washington, DC: National Highway Traffic Safety Administration.

Problem Examined: To identify and field test countermeasures to the pedestrian alcohol problem

Age Group(s) Addressed: Adults (15+ years)

Crash Type(s) Involved: Alcohol-related pedestrian crashes

Abstract: The objectives of this study were to analyze the pedestrian alcohol problem in a community and to develop and apply procedures to counter the problem. Baltimore, Maryland, was selected as the test city. It was estimated that approximately $40 \%$ of the pedestrian crashes in the city involved alcohol. An interdepartmental task force developed and implemented a comprehensive countermeasure program. When possible, countermeasures were implemented in one of two zones in the center of the city selected to include $73 \%$ of the pedestrian alcohol crashes in $21 \%$ of the land area. Since "had been drinking" was not routinely checked on police crash reports in the city, a surrogate measure was developed to estimate the occurrence of a pedestrian alcohol crash. This surrogate group included males between 30 and 59 who had pedestrian crashes from 7 p.m. to 3:59 a.m. on Thursday, Friday, Saturday, and Sunday nights (ending at 3:59 a.m. Monday morning). Substantial reductions for the surrogate group in total crashes, zone crashes, and crashes on roads on which special signs were erected lead to the conclusion that the study made positive inroads into reducing the pedestrian alcohol problem in Baltimore. In addition, a statistically significant time series analysis of crashes on treated roads involving males 14 and older lead to the conclusion that this was an effective pedestrian crash countermeasure. Also, the process for forming and using a community task force developed as part of this study formed the basis for the development of a guide for communities considering mounting pedestrian alcohol programs.

Related NHTSA Research: Blomberg, R.D., Preusser, D.F., Hale, A., \& Ulmer, R.G. A Comparison of Alcohol Involvement in Pedestrians and Pedestrian Casualties. Final Report, USDOT, DOT HS 805 521, October 1979. Washington, DC: National Highway Traffic Safety Administration.

Key study identifying and quantifying the alcohol-pedestrian problem.

Leaf, W.A., \& Preusser, D.F. Identification of Alcohol-Pedestrian Crash Problems Among Selected Racial/Ethnic Groups. Final Report, DOT HS 808 641, September 1997. Washington, DC: National Highway Traffic Safety Administration.

Further definition of the pedestrian alcohol problem. 
Title: Leaf, W.A., \& Preusser, D.F. Identification of Alcohol-Pedestrian Crash Problems Among Selected Racial/Ethnic Groups. Final Report, DOT HS 808 641, September 1997. Washington, DC: National Highway Traffic Safety Administration.

Problem Examined: People who have been drinking make up about half of all adult pedestrian crash fatalities. This study examined the extent of involvement of racial/ethic groups in this problem.

Age Group(s) Addressed: Adults (15 and older)

Crash Type(s) Involved: Alcohol-related pedestrian crashes

Abstract: About one-third of all adult pedestrian victims were at BACs of $.15 \mathrm{~g} / \mathrm{dL}$ or more (FARS, 1984-1993). This study examined racial/ethnic patterns of involvement in fatal crashes, then conducted focus group testing with members of at-risk populations to study cultural factors which might contribute to the alcohol-pedestrian problem and to study how countermeasures should be targeted for greatest effectiveness. Racial data was obtained for all 1987-89 FARS data and for 1 to 12 years of FARS data for seven States or State subsets. Analyses showed three specific groups with pedestrian-alcohol fatality risks as high as or higher than the population as a whole: Black adults 25 and older, Hispanic adult males, and Native American adults. Fourteen focus group discussions were conducted with Blacks, Hispanics, and Native Americans. Results were analyzed for cultural patterns of alcohol use and abuse, likely countermeasure mechanisms, and comments and suggestions on 28 specific countermeasure themes. Alcohol fatality rates and population values were calculated for 50 States, the District of Columbia, and 74 metropolitan areas. Recommendations were made for possible NHTSA follow-on countermeasure implementation tests.

Related NHTSA Research: Blomberg, R.D., Preusser, D.F., Hale, A., \& Ulmer, R.G. A Comparison of Alcohol Involvement in Pedestrians and Pedestrian Casualties. Final Report, USDOT, DOT HS 805 521, October 1979. Washington, DC: National Highway Traffic Safety Administration.

Key study identifying and quantifying the alcohol-pedestrian problem.

Blomberg, R.D., \& Cleven, A.M. Development, Implementation, and Evaluation of a Countermeasure Program for Alcohol-Involved Pedestrian Crashes. Final Report, DOT HS 809 067, July 2000. Washington, DC: National Highway Traffic Safety Administration. Project field tested techniques for reducing the alcohol-involved pedestrian crash problem. 


\title{
SPEED
}

Title: Leaf, W.A., \& Preusser, D.F. Literature Review on Vehicle Travel Speeds and Pedestrian Injuries. Final Report, DOT HS 809 021, October 1999. Washington, DC: National Highway Traffic Safety Administration.

Problem Examined: This study examined the association between vehicle travel speeds and pedestrian injuries. It also examined means of reducing speeds.

Age Group(s) Addressed: All ages

Crash Type(s) Involved: All pedestrian crash types

\begin{abstract}
The relationship between vehicle travel speeds and resulting pedestrian injury was reviewed in the literature and in existing data sets. Results indicated that higher vehicle speeds are strongly associated with both a greater likelihood of pedestrian crash occurrence and more serious resulting pedestrian injury. It was estimated that only $5 \%$ of pedestrians would die when struck by a vehicle traveling at $20 \mathrm{mph}$ or less. This compares with fatality rates of $40 \%, 80 \%$, and nearly $100 \%$ for striking speeds of 30, 40, and $50 \mathrm{mph}$ or more, respectively. Reductions in vehicle travel speeds can be achieved through lowered speed limits, police enforcement of speed limits, and associated public information. More long-lasting speed reductions in neighborhoods where vehicles and pedestrians commonly share the roadway can be achieved through engineering approaches generally known as traffic calming. Countermeasures include road humps, roundabouts, other horizontal traffic deflections (e.g., chicanes), and increased use of stop signs. Comprehensive community-based speed reduction programs, combining education, enforcement, and roadway engineering are recommended.
\end{abstract}

Related NHTSA Research: Blomberg, R.D., \& Cleven, A.M. Pilot Test of Heed the Speed, a Program to Reduce Speeds in Residential Neighborhoods, Final report, DOT HS 810648 , August 2006 . Washington, DC: National Highway Traffic Safety Administration. Conducted a field test of various countermeasures to the speed problem. 
Title: Blomberg, R.D., \& Cleven, A.M. Pilot Test of Heed the Speed, a Program to Reduce Speeds in Residential Neighborhoods, Final report, DOT HS 810 648, August 2006. Washington, DC: National Highway Traffic Safety Administration.

Problem Examined: Can an innovative program using public information, enforcement and special roadway markings combined with traffic calming achieve speed reductions obtained by traffic calming alone?

Age Group(s) Addressed: All ages

\title{
Crash Type(s) Involved: Not applicable
}

\begin{abstract}
There is abundant evidence that higher speeds are associated with more severe pedestrian injuries and increased death. Speeding is generally more dangerous for pedestrians on residential roads than on other roadways. There has been significant work on engineering approaches to traffic calming as a means to reduce neighborhood speeds. There have, however, been few attempts to combine public information, enforcement and innovative marking techniques with engineering changes as a means of achieving greater speed reductions. Some communities have used traffic calming on selected streets in a neighborhood but left others untouched because of objections voiced by emergency services. This has created streets within a defined calmed neighborhood where motorists continue to exceed prudent speeds - or at least exceed the speeds on adjacent streets. The objective of the current study was therefore to attempt to achieve on these untreated streets the level of speed reduction achieved on the adjacent streets that have received traffic calming treatments.
\end{abstract}

Three neighborhoods each in two cities, Phoenix and Peoria, Arizona, were selected for a Heed the Speed program based on the expressed desires of the residents to moderate vehicle speeds and/or a history of excessive speeding. Multiple roads were part of the study in some of the six areas. Neighborhood associations distributed education, including yard signs, pamphlets and letters to specific groups such as car dealers. The police increased enforcement patrols and tickets for egregious violations. They also added numerous warning stops for motorists exceeding the speed limit but below the range at which a ticket would normally be written. Speed tables or speed humps were added in two of the neighborhoods in the middle of the 3- to 6-month campaign. Innovative pavement markings that created the illusion of impediments were tried in three of the neighborhoods.

The program was evaluated by a pre/post mailed survey, by police data forms completed at each stop, and by multiple waves of speed measurements using on-road traffic counters. The survey showed a strong increase in knowledge of the program and the need to moderate speeds. Respondents also expressed a strong belief that speeds in their neighborhood had decreased since the Heed the Speed program was implemented. The police stop records showed that most violators were neighborhood residents. Speed measurements showed significant reductions in all six neighborhoods and on all test roads within the neighborhoods except one low-volume street with pre-existing speed humps installed. The baseline speeds on this street were already well below the prevailing $25 \mathrm{mph}$ speed limit and did not change significantly after the treatments. On all other treated roads, there were both a significant reduction in mean speed and a significant 
reduction in the percentage of vehicles doing $7 \mathrm{mph}$ or more above the speed limit. Mean speed reductions ranged from approximately $0.5 \mathrm{mph}$ to over $3.5 \mathrm{mph}$. The drop in the percentage of drivers exceeding the speed limit by $7 \mathrm{mph}$ or more ranged from about $15 \%$ to over $400 \%$.

Related NHTSA Research: Literature Review on Vehicle Travel Speeds and Pedestrian Injuries. Final Report, DOT HS 809 021, October 1999. Washington, DC: National Highway Traffic Safety Administration.

Provided an overview of the speed problem. 


\section{CHILDREN}

\section{Preschool}

Title: Phinney, J., Colker, L., \& Cosgrove, M. Literature Review on the Preschool Pedestrian. DOT HS 806 679, January 1985. Washington, DC: National Highway Traffic Safety Administration.

Problem Examined: The young preschool child is overrepresented in pedestrian crashes, and traffic crashes are the leading cause of death for children under 6 . A literature review of this problem area was done to facilitate the development of countermeasure programs for this age group.

Age Group(s) Addressed: Children under 6

Crash Type(s) Involved: Dart-out, midblock dash

Abstract: The purpose of this literature review was to describe (1) the factors leading to typical preschool pedestrian crashes, (2) the developmental characteristics of the preschool children that affect their behavior in traffic, (3) social factors that may place a preschooler at risk in traffic, (4) programs of traffic safety education for in-school and preschool use in the United States, and (5) preschool traffic safety programs developed by foreign countries. Detailed information relevant to each of these objectives is presented in this volume.

Related NHTSA Research: Applied Management Sciences, Inc., Development of a Preschool Child Pedestrian Traffic Safety Program: The Walking in Traffic Safely (WITS) Program for Preschoolers. Final Report. DOT HS 806 678, January 1985. Washington, DC: National Highway Traffic Safety Administration.

This follow-on project develops the preschool child pedestrian safety program. 
Title: Applied Management Sciences, Inc., Development of a Preschool Child Pedestrian Traffic Safety Program: The Walking in Traffic Safely (WITS) Program for Preschoolers. Final Report. DOT HS 806 678, January 1985. Washington, DC: National Highway Traffic Safety Administration.

Problem Examined: Preschoolers are at risk as pedestrians due to their very young age and lack of experience with the traffic situation. This project examined crash data for preschoolers and developed a training program for this age group.

Age Group(s) Addressed: Preschoolers up to age 5

Crash Type(s) Involved: Midblock dash, dart-out, backing vehicle, pedestrian in roadway, pedestrian not in roadway

Abstract: This report presents an overview of the process followed in developing the Walking in Traffic Safely (WITS) booklets for preschoolers, parents, and teachers. The key precepts promoted by the booklets are: preschoolers should not be allowed to cross the street by themselves; they should always be in the presence of an adult or older child; they begin by learning the names of parts of the environment (curb, street, sidewalk, etc.); identifying traffic boundaries; and learning the basics of crossing streets when with an adult. The steps involved in completing this project are described: (1) conduct of an in-depth review of the literature, (2) analysis of crash data involving preschool pedestrians, (3) identification of countermeasure ideas, (4) designation of an approach to curriculum design, and (5) development and testing of the curricular material. The concluding section of this report focuses on suggestions for further work. The WITS program was made available through the National Association for the Education of Young Children.

Related NHTSA Research: Phinney, J., Colker, L., \& Cosgrove, M. Literature Review on the Preschool Pedestrian. Literature Review, DOT HS 806 679, January 1985. Washington, DC: National Highway Traffic Safety Administration.

The literature review provided an overview of the field for the program developers. 


\section{CHILDREN}

\section{School Age - Urban}

Title: Hale, A., Blomberg, R.D., \& Preusser, D.F. Experimental Field Test of the Model Ice Cream Truck Ordinance In Detroit. Final Report, DOT HS 803 410, April 1978. Washington, DC: National Highway Traffic Safety Administration.

Problem Examined: To test the effectiveness of a legal countermeasure in reducing pedestrian crashes among children patronizing ice cream vendor trucks.

Age Group(s) Addressed: Children age 2 to 10

Crash Type(s) Involved: Vendor ice cream truck

Abstract: The Model Ice Cream Truck Ordinance (MICTO) is a legal countermeasure designed to prevent child pedestrian crashes from occurring near ice cream trucks. Among other things, the MICTO requires motorists to stop before passing an ice cream truck displaying the special swing arm and flashing lights which must be actuated when the truck is stopped to vend.

The MICTO was enacted by Detroit on May 12, 1976, and became fully effective on June 10, 1976. A two-year field test of the safety-effectiveness of the MICTO was conducted. Results for 1976 show that radar measured average motorist speed abreast of vending ice cream trucks was reduced from $28.10 \mathrm{mph}$ before the MICTO to $15.65 \mathrm{mph}$ after the MICTO - a $44 \%$ reduction. During the July 1- October 31, 1976, period, vendor-related child pedestrian crashes were reduced from a prior three-year average of 19.67 before the MICTO to 9 after the MICTO - a 54\% reduction. In 1977 average motorist speed at the truck continued at a reduced level of $15.79 \mathrm{mph}$. However, vendor-related child pedestrian crashes were lowered from a 1973-75 preMICTO average of 48.67 to 11 in 1977 - a 77\% reduction.

A pamphlet explaining the Model Ice Cream Truck Ordinance is available from NHTSA, NTI131, 1200 New Jersey Avenue SE., Washington, DC 20590.

Related NHTSA Research: Berger, W. G. Urban Pedestrian Accident Countermeasures Experimental Evaluation: Volume I-Behavioral Evaluation Study. Final Report, DOT HS 801 346, February 1975. Washington, DC: National Highway Traffic Safety Administration. Tested an early version of a vendor countermeasure.

Blomberg, R.D., Hale, A., \& Kearney, E.F. Development of Model Regulations for Pedestrian Safety. Final Report. DOT HS 801 287, November 1974. Washington, DC: National Highway Traffic Safety Administration.

Created the model ice cream truck ordinance. 
Title: Thackray, R.M., Jr., \& Dueker, R.L. Child Pedestrian Supervision/Guidance. Final Report. DOT HS 806 519, January 1983. Washington, DC: National Highway Traffic Safety Administration.

Problem Examined: Preschool and young school-age children are heavily victimized by autopedestrian crashes. Prior NHTSA research indicated that the lack of parental supervision was a major predisposing factor for many of these crashes. This study looked at children at play and recommended various supervision/guidance countermeasures.

Age Group Addressed: Preschool children, early school-age children

Crash Type(s) Involved: Dart-out, playing in roadway, play vehicle related

\begin{abstract}
The purpose of this study was to identify and evaluate the pedestrian crash risk associated with play activities performed in and near the street, as well as other situational characteristics, and to develop crash countermeasures emphasizing supervision and guidance. The primary focus of the study was on pre-school children, but early school-age children were also studied.
\end{abstract}

Behavioral observations of children at play on/near the street were conducted in five U.S. cities. Areas chosen for observation had moderate to high child pedestrian crash frequency. Interviews were conducted with a subsample of the children observed and their parents.

Several risk measures were evaluated by comparing magnitude of risk values derived from the observation data for various play activities against the frequency of pedestrian crashes involving these play activities. Inadequate search street entries, child-vehicle conflicts, and time observed in-street were the risk measures selected. Results included the identification of high risk play activities and evaluation of risk as a function of type of area, selected demographic factors, time of day and day of week.

Nine supervision/guidance countermeasures were identified and an implementation model was specified. A plan for evaluating the countermeasures and implementation model was developed.

Related NHTSA Research: Applied Management Sciences, Inc., Development of a Preschool Child Pedestrian Traffic Safety Program: The Walking in Traffic Safely (WITS) Program for Preschoolers. Final Report. DOT HS 806 678, January 1985. Washington, DC: National Highway Traffic Safety Administration.

Provide a training program for preschool pedestrians that stresses adult supervision.

Phinney, J., Colker, L., \& Cosgrove, M. Literature Review on the Preschool Pedestrian. Literature Review, DOT HS 806 679, January 1985. Washington, DC: National Highway Traffic Safety Administration.

Surveys many safety programs and the scientific literature dealing with the young child. 
Title: Dueker, R.L. Threat Detection Training Programs for Child Pedestrian Safety. Volume I: Conduct, Results and Recommendations. Final Report, DOT HS 801 450, March 1975.

Washington, DC: National Highway Traffic Safety Administration.

Problem Examined: How to develop a training program to reduce dart-out crashes among young pedestrians.

Age Group(s) Addressed: Kindergarten-to-third graders in urban schools

Crash Type(s) Involved: Dart-out, midblock dash

Abstract: Research aim was to identify efficient, safe, street-crossing behavior sequence for children; develop/pilot-test programs to train sequence in public schools. Target audience: Kindergarten-to-third graders in urban schools. Phase I work included behavioral analysis to find optimum street crossing behavior sequence; evaluate behavior-modification techniques; investigate constraints (cost, school resource limitations, school personnel training/attitudes).

Phase II developed three programs evaluated for adequacy, feasibility, cost by project staff, consultants, six school principals, 11 teachers: (1) Basic Program - teacher introduces program, behavior sequence; children practice sequence through games played on lo-fi simulated classroom "street" and later on real streets (traffic blocked). (2) Film Program - same as Basic, except program, behavior sequence introduced via film starring TV's Captain Kangaroo. (3) Simulator Program - same as Basic, except children practice sequence in hi-fi classroom simulator, using two rear-projected synchronized color films of moving traffic.

Phase III pilot-tested training programs in three urban Pittsburgh schools re effectiveness, permanency, training-material adequacy, using one pretest, two posttests. All three programs considerably reduced unsafe street-crossing behavior. The Film Program and Simulator Program were generally superior to the Basic Program; however, their was little difference between film and simulator programs in effectiveness and permanency. It was recommended that the film and simulator programs undergo further testing.

Related NHTSA Research: Blomberg, R.D., \& Preusser, D.F. Identification and Test of Pedestrian Safety Messages for Public Education Programs. Final Report. DOT HS 801 457, March 1975. Washington, DC: National Highway Traffic Safety Administration.

Provides an alternate approach to same problem via safety messages.

Dueker, R.L. Experimental Field Test of Proposed Anti-Dart-Out Training Programs. Volume 1: Conduct and Results. Final Report, DOT HS 806 195, December 1981. Washington, DC: National Highway Traffic Safety Administration.

Tests the anti-dart-out training program for its crash-reduction capabilities. 
Dueker, R.L., \& Berger, S.S. Experimental Field Test of Proposed Anti-Dart-Out Training Programs. Volume 2: Implementation Guidelines and Program Materials. Final Report, DOT HS 806 196, December 1981. Washington, DC: National Highway Traffic Safety Administration.

Provides users guide for school systems implementing the anti-dart-out training program.

Dueker, R.L., \& Berger, S.S. Field Test of Proposed Anti-Dart-Out Training Programs, Volume 3: Program Staff Training Materials and Videotape/Film. Final Report, DOT HS 806 197, December 1981. Washington, DC: National Highway Traffic Safety Administration.

Provides staff training materials and scripts for anti-dart-out training program. 
Title: Dueker, R.L. Experimental Field Test of Proposed Anti-Dart-Out Training Programs. Volume 1: Conduct and Results. Final Report, DOT HS 806 195, December 1981. Washington, DC: National Highway Traffic Safety Administration.

Problem Examined: The major cause of death and injury to young children is the dart-out crash. This project developed and evaluated a safe-street-crossing training program for 5- to 9-year-olds designed to combat this crash type.

Age Group(s) Addressed: 5- to 9-year-olds.

Crash Type(s) Involved: Dart-out, midblock dash

Abstract: This report describes the conduct and results of an evaluation of a child pedestrian anti-dart-out training program. Two versions were tested: A film program and a film/simulator program. Before/after crash and street crossing behavior data were collected in one city for each program, and in a comparison city. Crash data was collected in two additional comparison cities. Data on student/teacher reactions and deviations in the conduct of the program was also collected. The film program was found to be superior in reduction of unsafe street-crossing behavior and on various practicality considerations. Although traditional levels of statistical significance were not obtained in the main analyses, there was statistical and intuitive evidence to support the effectiveness of the film program in the reduction of crashes among the children exposed to the program. Teacher attitudes toward the film/ simulator program were initially superior, although this difference lessened over time. Student attitudes were generally positive. Deviations were numerous but serious in only a few cases concerning the film/simulator program. The film program was recommended for further development/implementation. Discussions of results and program improvements are provided.

Related NHTSA Research: Blomberg, R.D., Preusser, D.F., Hale, A., \& Leaf, W.A. Experimental Field Test of Proposed Pedestrian Safety Messages: 3 Volumes: Volume II: Child Messages, Final Report, DOT HS 806 522, November 1983. Washington, DC: National Highway Traffic Safety Administration.

This project tested an alternate countermeasure approach to the same problem. 
Title: Dueker, R.L., \& Berger, S.S. Field Test of Proposed Anti-Dart-Out Training Programs, Volume 2. Implementation Guidelines and Program Materials. Final Report, DOT HS 806 196, December 1981. Washington, DC: National Highway Traffic Safety Administration.

Problem Examined: The major cause of death and injury to young children is the dart-out crash. This project developed and evaluated a safe-street-crossing training program for 5 to 9-year-olds designed to combat this crash type.

Age Group(s) Addressed: 5- to 9-year-olds.

Crash Type(s) Involved: Dart-out, midblock dash

Abstract: This report (Volume 2 of three volumes) provides detailed descriptions of all program material employed with the recommended version of a child pedestrian safety program. Volume I of this report describes the conduct and results of the evaluation of two alternate child pedestrian anti-dart-out training programs and provides recommendations concerning the more effective program. The material in Volume 2 have been revised in accordance with the recommendations for modification of this program and its material provided in Volume 1.

This volume is designed to serve as a users guide for school systems implementing the program. It provides complete, organized information on every aspect of the program. Implementation guidelines are provided and issues of concern to users are discussed., The full texts of the program guides which specify program content and conduct are provided. Copies of all other printed material are included. Specifications for other program material are provided and possible alternatives are discussed. Several additional material have been recommended for use in the program. These material, training programs for the program coordinator and instructors and a script of an introductory videotape, are provided in Volume 3.

Related NHTSA Research: Blomberg, R.D., Preusser, D.F., Hale, A., \& Leaf, W.A. Experimental Field Test of Proposed Pedestrian Safety Messages: 3 Volumes: Volume II: Child Messages, Final Report, DOT HS 806 522, November 1983. Washington, DC: National Highway Traffic Safety Administration.

This project tested an alternate countermeasure approach to the same problem. 
Title: Dueker, R.L., \& Berger, S.S. Field Test of Proposed Anti-Dart-Out Training Programs, Volume 3: Program Staff Training Materials and Videotape/Film. Final Report, DOT HS 806 197, December 1981. Washington, DC: National Highway Traffic Safety Administration.

Problem Examined: The major cause of death and injury to young children is the dart out crash. This project developed and evaluated a safe street crossing training program for 5 to 9 -year-olds designed to combat this crash type.

Age Group(s) Addressed: 5- to 9-year-olds.

Crash Type(s) Involved: Dart-out, midblock dash

Abstract: This report (Volume 3 of three volumes) provides detailed descriptions of additional program material suggested for use with the recommended version of a child pedestrian safety program. Volume 1 of this report describes the conduct and results of the evaluation of two alternate child pedestrian anti-dart out training programs and provides recommendations concerning the more effective program.

Volume 2 is a user's guide for school systems implementing the program. Implementation guidelines and the full texts of the program guides specifying program content and conduct are provided.

This volume contains additional material and videotape/film scripts which have been recommended for use in the program. Training programs for the program coordinators and instructors are included, in addition to a program introductory videotape/film and videotape/film training vignettes for use in personnel training.

Related NHTSA Research: Blomberg, R.D., Preusser, D.F., Hale, A., \& Leaf, W.A. Experimental Field Test of Proposed Pedestrian Safety Messages: 3 Volumes: Volume II: Child Messages, Final Report, DOT HS 806 522, November 1983. Washington, DC: National Highway Traffic Safety Administration.

This project tested an alternate countermeasure approach to the same problem. 


\section{CHILDREN}

\section{School Age - Rural}

Title: Dueker, R. L., \& Chiplock, L.W. Identification and Feasibility Test of Specialized Rural Pedestrian Safety Training. Volume I: Program Development and Training. Final Report. DOT HS 806 256, March 1981. Washington, DC: National Highway Traffic Safety Administration.

Problem Examined: A pedestrian safety training program was developed to combat pedestrian crashes victimizing suburban and rural children.

Age Group(s) Addressed: Kindergarten through 12th grade children.

Crash Type(s) Involved: Midblock dash, dart-out, vendor - ice cream truck, mailbox-related, school bus-related, intersection dash, multiple threat, walking along roadway, hitchhiking.

Abstract: This report describes the development and evaluation of a K-12 pedestrian safety curriculum for suburban and rural schools. The three-program curriculum, called PedSafe, was developed to combat pedestrian crashes which victimize suburban/rural children. The Elementary and On-Bus Programs were designed to train safe behaviors in four pedestrian situations - midblock crossing, intersection crossing, walking along the roadway, and pedestrian movement near the school bus. The Junior/Senior High School Program teaches pedestrian safety principles. Statistically significant reductions in unsafe pedestrian behavior brought about by the Elementary and On-Bus Programs were found, using a before-after with control design. A similar design was employed to evaluate pedestrian safety knowledge gain for the Junior/Senior High School Program. Statistically significant gains resulted from all five units within this program.

The report consists of four volumes. Volume 1 describes PedSafe development and the evaluation results. Volumes 2 and 3 provide copies of revised text material for, respectively, the Elementary and On-Bus Programs and the Junior/Senior High School Program. The final volume, Volume 4, provides audiovisual scripts.

Related NHTSA Research: Chiplock, L.,W., \& Dueker, R.L. Identification and Feasibility Test of Specialized Rural Pedestrian Safety Training. Volume II: PedSafe Elementary Materials.

Final Report, DOT HS 805 964, March 1981. Washington, DC: National Highway Traffic Safety Administration.

Other volume in this series of reports.

Bittner, S.R., Chiplock, L. W., \& Dueker, R.L. Identification and Feasibility Test of Specialized Rural Pedestrian Safety Training. Volume III: Pedsafe Junior/Senior High School Materials. Final Report, DOT HS 805 965, March 1981. Washington, DC: National Highway Traffic Safety Administration.

Other volume in this series of reports. 
Chiplock, L.W., Dueker, R.L., \& Bittner, S.R. Identification and Feasibility Test of Specialized Rural Pedestrian Safety Training. Volume IV: Pedsafe Audiovisual Scripts. Final Report, DOT HS 805 966, March 1981. Washington, DC: National Highway Traffic Safety Administration. Other volume in this series of reports. 
Title: Chiplock, L.,W., \& Dueker, R.L. Identification and Feasibility Test of Specialized Rural Pedestrian Safety Training. Volume II: PedSafe Elementary Materials. Final Report, DOT HS 805 964, March 1981. Washington, DC: National Highway Traffic Safety Administration.

Problem Examined: A pedestrian safety training program was developed to combat pedestrian crashes victimizing suburban and rural children.

Age Group(s) Addressed: Kindergarten through 12th grade children.

Crash Type(s) Involved: Midblock dash, dart-out, vendor - ice cream truck, mailbox-related, school-bus-related, intersection dash, multiple threat, walking along roadway, hitchhiking.

Abstract: This report (Volume 2 of four volumes) serves as a users' guide for elementary schools implementing the PedSafe Program. Volume 1 of this report describes the conduct and results of the evaluation of the entire PedSafe Program and provides recommendations concerning revisions in material. Volume 3 provides all printed program material employed in the Junior/Senior High School PedSafe Program. The material in Volumes 2 and 3 has been revised in accordance with the recommendations for modification of this program and its material provided in Volume 1. Volume 4 contains the scripts for all audiovisuals used in the PedSafe Program.

This volume contains all printed material used in the Elementary and On-Bus PedSafe Programs, including Principal's Guide, Teacher's Guides, parent's pamphlets, Transportation Director's Manual, and Bus Driver's Manual.

Related NHTSA Research: Dueker, R. L., \& Chiplock, L.W. Identification and Feasibility Test of Specialized Rural Pedestrian Safety Training. Volume I: Program Development and Training. Final Report. DOT HS 806 256, March 1981. Washington, DC: National Highway Traffic Safety Administration.

Other volume in this series of reports.

Bittner, S.R., Chiplock, L. W., \& Dueker, R.L. Identification and Feasibility Test of Specialized Rural Pedestrian Safety Training. Volume III: Pedsafe Junior/Senior High School Materials. Final Report, DOT HS 805 965, March 1981. Washington, DC: National Highway Traffic Safety Administration.

Other volume in this series of reports.

Chiplock, L.W., Dueker, R.L., \& Bittner, S.R. Identification and Feasibility Test of Specialized Rural Pedestrian Safety Training. Volume IV: Pedsafe Audiovisual Scripts. Final Report, DOT HS 805 966, March 1981.1981. Washington, DC: National Highway Traffic Safety Administration.

Other volume in this series of reports.

Cleven, A. M., and Blomberg, R.D. Development and Evaluation of a Pedestrian Safety Training Program for Elementary School Bus Rider. Final Report. DOT HS 808 267, December 1994. Washington, DC: National Highway Traffic Safety Administration. 
Developed new school bus program which was made available from the National Safety Council. 
Title: Bittner, S.R., Chiplock, L. W., \& Dueker, R.L. Identification and Feasibility Test of Specialized Rural Pedestrian Safety Training. Volume III: Pedsafe Junior/Senior High School Materials. Final Report, DOT HS 805 965, March 1981. Washington, DC: National Highway Traffic Safety Administration.

Problem Examined: A pedestrian safety training program was developed to combat pedestrian crashes victimizing suburban and rural children.

Age Group(s) Addressed: Kindergarten through 12th grade children.

Crash Type(s) Involved: Midblock dash, dart-out, vendor - ice cream truck, mailbox-related, school-bus-related, intersection dash, multiple threat, walking along roadway, hitchhiking.

Abstract: This report (Volume 3 of four volumes) provides detailed descriptions of all printed program material employed in the Junior/Senior High School PedSafe Program. Volume 1 of this report describes the conduct and results of the evaluation of the entire PedSafe Program and provides recommendations concerning material revisions. Volume 2 is a users' guide for elementary schools implementing the program. The material in Volume 3 have been revised in accordance with the recommendations for modification of this program and its material provided in Volume 1. Volume 4 contains the scripts for all audiovisuals used in the PedSafe Program.

This volume is designed to serve as a users guide for school systems implementing the program. The full texts of the PedSafe Teacher's Guides and Student Booklets which specify program content and conduct are provided. Copies of all other printed material are included. In addition, specifications for printing program material are provided. Pre- and post-knowledge tests are also included.

Related NHTSA Research: Dueker, R. L., \& Chiplock, L.W. Identification and Feasibility Test of Specialized Rural Pedestrian Safety Training. Volume I: Program Development and Training. Final Report. DOT HS 806 256, March 1981. Washington, DC: National Highway Traffic Safety Administration.

Other volume in this series.

Chiplock, L.,W., Dueker, R.L., \& Bittner, S.R. Identification and Feasibility Test of Specialized Rural Pedestrian Safety Training. Volume II: PEDSAFE Elementary Materials. Final Report, DOT HS 805 964, March 1981. Washington, DC: National Highway Traffic Safety

Administration.

Other volume in this series.

Chiplock, L.W., Dueker, R.L., \& Bittner, S.R. Identification and Feasibility Test of Specialized Rural Pedestrian Safety Training. Volume IV: Pedsafe Audiovisual Scripts. Final Report, DOT HS 805 966, March 1981. Washington, DC: National Highway Traffic Safety Administration. Other volume in this series. 
Title: Chiplock, L.W., Dueker, R.L., \& Bittner, S.R. Identification and Feasibility Test of Specialized Rural Pedestrian Safety Training. Volume IV: Pedsafe Audiovisual Scripts. Final Report, DOT HS 805966, March 1981. Washington, DC: National Highway Traffic Safety Administration.

Problem Examined: A pedestrian safety training program was developed to combat pedestrian crashes victimizing suburban and rural children.

Age Group(s) Addressed: Kindergarten through 12th grade children.

Crash Type(s) Involved: Midblock dash, dart-out, vendor - ice cream truck, mailbox-related, school-bus-related, intersection dash, multiple threat, walking along roadway, hitchhiking.

Abstract: This report (Volume 4 of four volumes) provides the scripts for all audiovisuals employed in the PedSafe Program. Volume 1 of this report describes the conduct and results of the evaluation of the entire PedSafe Program and provides recommendations concerning materials revisions. Volumes 2 and 3 are users' guides for elementary and junior/senior high schools, respectively, implementing the program.

The audiovisuals produced for the PedSafe Program were of research quality (i.e., professional actors were not employed, camera equipment was of average quality, etc.), although the quality was such that a meaningful pilot test could be conducted. Since all audiovisuals will have to be produced professionally for full-scale implementation, this volume has been subdivided into classes of audiovisuals: those needing few script/visual modifications and those needing major modifications.

Related NHTSA Research: Dueker, R. L., \& Chiplock, L.W. Identification and Feasibility Test of Specialized Rural Pedestrian Safety Training. Volume I: Program Development and Training. Final Report. DOT HS 806 256, March 1981. Washington, DC: National Highway Traffic Safety Administration.

Other volume in this series.

Chiplock, L.,W., \& Dueker, R.L. Identification and Feasibility Test of Specialized Rural Pedestrian Safety Training. Volume II: PEDSAFE Elementary Materials. Final Report, DOT HS 805 964, March 1981. Washington, DC: National Highway Traffic Safety Administration. Other volume in this series.

Bittner, S.R., Chiplock, L. W., \& Dueker, R.L. Identification and Feasibility Test of Specialized Rural Pedestrian Safety Training. Volume III: Pedsafe Junior/Senior High School Materials. Final Report, DOT HS 805 965, March 1981. Washington, DC: National Highway Traffic Safety Administration.

Other volume in this series. 


\section{OLDER (65+) ADULTS}

Title: Blomberg, R.D., Cleven, A. M., \& Edwards, J. M. Development of Safety Information Materials and Media Plans for Elderly Pedestrians. Final Report, DOT HS 808 132, June 1993. Washington, DC: National Highway Traffic Safety Administration.

Problem Examined: Older adults are particularly vulnerable as pedestrians. Although they have fewer crashes $(7.7 \%)$ than would be expected by their numbers in the population $(12.5 \%)$, they have almost one-quarter $(22.7 \%)$ of all pedestrian fatalities. This study focuses on the pedestrian risks of older adults and ways of safeguarding older pedestrians.

Age Group(s) Addressed: Adults 65 and older

Crash Type(s) Involved: Vehicle turn/merge, other intersection crashes, backing vehicle, conspicuity enhancement

Abstract: The objectives of this study were to: (1) develop pedestrian safety messages which will have a countermeasure effect on the specific types of crashes occurring to older pedestrians and (2) develop media plans for use by NHTSA in disseminating the information via various organizations. Prior research was reviewed and several recent crash data sets were obtained and analyzed to identify the types of crashes in which older adults are involved. This effort resulted in identification of the following crash type groupings: crashes involving vehicles turning at an intersection including Vehicle Turn/Merge and Turning Vehicle; "Other Intersection" crash types which do not involve turning movements; and the "Backing" crash type. In addition, it was confirmed that older-adult crashes increase markedly in the winter months when the sun angle is lowest. This increase appears to be a problem of conspicuity. Pedestrian safety messages were then developed for each of the four defined situations. The risks and behavioral advice were documented in a paper entitled Walking Through the Years intended as a reference document for potential distributors of the proposed countermeasures. Additional supporting material included a slide series and presenter's guide to convey study results both to potential distribution organizations and to groups of older adults and a flyer version of the same material prepared for direct distribution to older adult target audiences. Organizations capable of reaching large numbers of older people were then identified and plans for use by NHTSA in disseminating the information were prepared

Related NHTSA Research: Blomberg, R.D., \& Cleven, A.M. Development, Implementation and Evaluation of a Pedestrian Safety Zone for Elderly Pedestrians. Final Report, DOT HS 808 692, February 1998. Washington, DC: National Highway Traffic Safety Administration. Project used this safety material in a field study. 
Title: Blomberg, R.D., \& Cleven, A.M. Development, Implementation and Evaluation of a Pedestrian Safety Zone for Elderly Pedestrians. Final Report, DOT HS 808 692, February 1998. Washington, DC: National Highway Traffic Safety Administration.

Problem Examined: How can safety countermeasures most efficiently be applied to olderpedestrian crashes?

Age Group(s) Addressed: Adults 65 and older

Crash Type(s) Involved: Older-adult pedestrian crash types

\begin{abstract}
America's population is aging, and the effects of this development will ripple through many aspects of our society. As pedestrians, older citizens present a special problem. Although the group is under-represented in the pedestrian flow and has an extremely low injury rate, it has the highest pedestrian fatality rate among all ages. This is typically attributed to a frailty factor. That is, older people tend to die in crashes which would be survivable by younger pedestrians. This project was a joint NHTSA/FHWA study designed to develop and apply procedures for defining pedestrian safety zones within communities, and to develop, implement and evaluate a set of countermeasures aimed at reducing the older-adult pedestrian crashes in the zones. Zone definition procedures were developed and applied to two cities: Phoenix and Chicago. Countermeasure development included a comprehensive video, 5 public service announcements and 13 flyers. Other available NHTSA and AAA education countermeasures were used in the study. A detailed engineering evaluation of each zone resulted in the selection of engineering countermeasures for the two cities. Extensive countermeasure programs were implemented in both cities. A complete evaluation was conducted only for the city of Phoenix. Data from Phoenix showed significant reductions in in-zone crashes to 65 -amd-older pedestrians over a period in which the city's population and overall pedestrian crashes increased. It was concluded that the zone process resulted in an effective and efficient means of deploying pedestrian countermeasures. A separate document describes procedures that city planners can use in defining zones and applying the zone process to pedestrian safety.
\end{abstract}

Related NHTSA Research: Blomberg, R.D., Cleven, A. M., \& Edwards, J. M. Development of Safety Information Materials and Media Plans for Elderly Pedestrians. Final Report, DOT HS 808 132, June 1993. Washington, DC: National Highway Traffic Safety Administration. Identified specific risks for older pedestrians and developed media material providing safety advice. 


\section{APPENDIX B - PEDESTRIAN AND BICYCLIST CRASH TYPES APPENDIX B}

PEDESTRIAN AND BICYCLIST CRASH TYPES

- Snyder and Knoblauch (1971) Pedestrian Crash Types

- NHTSA (1983) Pedestrian Crash Types

- Harkey et al. (2000) Pedestrian and Bicycle Crash Analysis Tool (PBCAT) Pedestrian Crash Types

- Cross and Fisher (1977) Bicyclist Crash Types

- Harkey et al. (2000) Pedestrian and Bicycle Crash Analysis Tool (PBCAT) Bicyclist Crash Types

Snyder and Knoblauch (1971) Pedestrian Crash Types

\begin{tabular}{|l|}
\hline Dart-outs and dashes \\
Dart-out first half \\
Dart-out second half \\
Pedestrian strikes vehicle \\
Intersection dash \\
\hline Other typical pedestrian situations \\
Multiple threat situation \\
Pedestrian waiting to cross in roadway \\
Vehicle turn/merge with attention conflict \\
Multiple pedestrian split \\
Situations with specific predisposing factors \\
Vendor-ice cream truck \\
Pedestrian exiting from vehicle \\
Bus stop related \\
Backing up \\
\hline
\end{tabular}




\begin{tabular}{|l|}
\hline Non-street locations \\
Non-pedestrian activity not in roadway \\
Freeway-expressway-from car \\
Freeway-expressway-crossing \\
Off-street parking \\
Atypical pedestrian activity \\
Non-pedestrian activity in roadway \\
Pedestrian walking in roadway \\
Working on vehicle \\
Rear wheel-truck or bus \\
Weird \\
\hline Miscellaneous \\
Precipitated by illegal antisocial act to pedestrian \\
Hot pursuit \\
Result of auto-auto-crash \\
Driverless vehicle \\
\hline Atypical causes-not pedestrian countermeasure corrective \\
Inadequate information-non-fatal \\
Inadequate information-fatal \\
Pedestrian operating bike or cart
\end{tabular}




\section{NHTSA (1983) Pedestrian Crash Types}

\section{Category 1: Bus stop, vendor, mailbox and vehicle exiting/entering crashes}

110 Commercial-bus-related - The pedestrian was struck while crossing in front of a commercial bus standing at a marked bus stop.

120 School-bus-related - The pedestrian was struck going to/from a school bus or school bus stop.

130 Vendor/ice cream truck - The pedestrian was struck while going to/from an ice cream or other vendor and was struck by a vehicle passing the vendor.

140 Mailbox-related - The pedestrian was struck while going to or from a private residence mailbox or newspaper box.

150 Exiting/entering parked vehicle - The pedestrian was struck while in the process of exiting or entering a parked or stopped vehicle and was struck in the traffic lane next to the parked or stopped vehicle.

\section{Category 2: Driverless, backing and pursuit crashes}

210 Driverless vehicle - The pedestrian was struck by a vehicle that was moving without a driver at the controls or which was set into motion by the actions of a child.

220 Backing vehicle - The pedestrian was struck by a vehicle that was backing up and, prior to impact, the pedestrian was not aware that the vehicle was backing.

230 Hot pursuit - The pedestrian was struck by a vehicle on an emergency or police mission, or by a vehicle being pursued (does not include a pedestrian who is pursuing or being pursued).

\section{Category 3: Disabled and emergency vehicle crashes}

310 Walking to or from disabled vehicle - The pedestrian was struck while walking along the roadway to or from a disabled vehicle (e.g., to get help, gas, etc.) but not in the immediate vicinity of the disabled vehicle.

320 Disabled-vehicle-related - The pedestrian was struck while working on or standing near a disabled vehicle in or along the roadway (no emergency vehicles present).

330 Emergency/police-vehicle-related - The pedestrian was struck while near an active emergency or police vehicle.

\section{Category 4: Working and playing in roadway crashes}

410 Working on roadway - The pedestrian (e.g., police or emergency personnel, flagman, traffic guard, or member of roadway construction or maintenance crew) was struck while working on, in, over, or under the roadway.

420 Play-vehicle-related - The pedestrian was struck while riding a play vehicle (e.g., wagon, sled, skateboard; but NOT a bicycle, "Big Wheel" type vehicle, or tricycle). 
430 Playing in roadway - The pedestrian was struck while playing on foot in the roadway. The pedestrian was playing in the roadway prior to the vehicle's appearance.

\section{Category 5: Hitchhiking, expressway-crossing and walking in roadway crashes}

510 Hitchhiking - The pedestrian was struck while hitchhiking.

520 Expressway-crossing - The pedestrian was struck while attempting to cross a limited access expressway.

531 Walking along road with traffic - The pedestrian was struck while walking or running along a roadway in the same direction as traffic.

532 Walking along road against traffic - The pedestrian was struck while walking or running along a roadway facing traffic.

533 Walking along road but direction with respect to traffic is unknown - The pedestrian was struck while walking or running along a roadway but direction with respect to traffic is unknown.

\section{Category 6: Non-roadway crashes}

610 Pedestrian waiting to cross at/near curb - The pedestrian was struck while standing at or near the curb or edge of roadway, waiting to cross the roadway.

620 Pedestrian not in roadway - The pedestrian was struck when not in or near a roadway (e.g., pedestrian was in a parking lot, driveway, private road, gas station, alley, sidewalk, yard, garage, or ball field).

\section{Category 7: Intersection crashes}

710 Multiple threat at intersection - The pedestrian crossed roadway at intersection in front of stopped or standing traffic, and was struck by a vehicle heading in the same direction as the stopped traffic.

720 Vehicle turn/merge - The pedestrian and vehicle collided while the vehicle was in the process of turning/merging, was preparing to turn/merge, or had just completed a turning/ merging maneuver.

730 Intersection dash - The motorist's view of the pedestrian was blocked until an instant before impact and/or the pedestrian was running.

740 Trapped - At a signalized intersection, a pedestrian in the process of crossing was struck when the light changed and traffic started moving.

750 Pedestrian walks into vehicle at intersection - The pedestrian walked (not ran) into (i.e., struck) the vehicle.

760 Intersection-driver violation - The pedestrian was struck by a driver who was proceeding straight ahead and the driver committed one or more of the following violations: careless driving, failure to yield right-of-way, signal/sign violation, speeding/too fast for conditions, DWI/DUI.

790 Intersection-other - The crash occurred at an intersection but is not covered by any of the above crash types or there is insufficient information to code the crash in any of the above crash types. 


\section{Category 8: Midblock crashes}

810 Multiple threat-midblock - The pedestrian entered the roadway midblock in front of stopped or standing traffic and was struck by a vehicle heading in the same direction as stopped traffic (driver's view of the pedestrian was blocked by standing traffic).

821 Midblock dart out (first half) - The pedestrian entered the roadway midblock and was struck before crossing half of the roadway (the driver's view of the pedestrian was blocked until an instant before impact).

822 Midblock dart out (second half) - The pedestrian entered the roadway midblock and was struck after crossing half of the roadway (the driver's view of the pedestrian was blocked until an instant before impact).

829 Midblock dart out (can't specify) - The pedestrian entered the roadway midblock and was struck (the driver's view of the pedestrian was blocked until an instant before impact) (first or second half of the roadway was not specified).

830 Midblock dash - The pedestrian ran into the roadway midblock and the motorist's view of the pedestrian was not obstructed.

840 Pedestrian walks into vehicle midblock - The pedestrian walked into (i.e., struck) the vehicle midblock.

890 Midblock-other - The crash occurred midblock but is not covered by any of the other crash types or there is insufficient information to use any of the above codes.

\section{Category 9: Other crashes or inadequate information}

910 Other-weird - The crash is not covered by any of the types listed in categories 1-8.

920 Inadequate information - There is insufficient information available to specify the crash type. 


\section{Harkey et al. (2000) PBCAT Pedestrian Crash Types}

\section{Unusual circumstances (100)}

110 Assault with vehicle - The driver intentionally struck the pedestrian with the vehicle

120 Dispute-related - The pedestrian was struck by a vehicle during a domestic altercation or other dispute

130 Pedestrian on vehicle - The pedestrian was sitting on, leaning against, or clinging to a vehicle which began to move or was moving.

140 Vehicle-vehicle/object - The pedestrian was struck as a result of a prior vehicleinto-vehicle or vehicle-into-object crash.

190 Other - unusual - The crash involved other unusual circumstances, such as a pedestrian being struck by falling cargo or a loose wheel.

\section{Unique vehicle type/vehicle action (200)}

211 Backing vehicle - driveway - The pedestrian was struck in a driveway by a vehicle that was backing with a driver at the controls."

212 Backing vehicle - driveway/sidewalk intersection - The pedestrian was struck in a driveway/sidewalk intersection by a vehicle that was backing with a driver at the controls.

213 Backing vehicle - roadway - The pedestrian was struck in a roadway by a vehicle that was backing with a driver at the controls.

214 Backing vehicle - parking lot - The pedestrian was struck in a parking lot by a vehicle that was backing with a driver at the controls.

219 Backing vehicle - other/unknown - The pedestrian was struck in another or unknown location by a vehicle that was backing with a driver at the controls.

220 Driverless vehicle - The pedestrian was struck by a vehicle that was moving without a driver at the controls or was set in motion by the actions of a child.

230 Disabled vehicle-related - The pedestrian was struck while near, next to, or while walking to or from a disabled vehicle (including a vehicle that had been in a crash); also include pedestrians standing near tow trucks responding to the disabled vehicle.

240 Emergency vehicle-related - The pedestrian was struck while near an active emergency vehicle, by an active emergency vehicle, or by a vehicle being pursued.

250 Play-vehicle related - The pedestrian was struck while riding a play vehicle that was not a bicycle (e.g., wagon, sled, tricycle, skates, big wheel, etc.).

\section{Unique pedestrian action (300)}

311 Working in roadway - The pedestrian was working in the roadway as part of their job when struck.

312 Playing in roadway - The pedestrian was playing in the roadway when struck.

313 Lying in roadway The pedestrian was lying in the roadway when struck. 
320 Exiting/entering parked vehicle - The pedestrian was getting into or out of a stopped or parked vehicle.

331 Mailbox-related - crossing roadway - The pedestrian was going to/from a mailbox or newspaper box and was struck while crossing the roadway.

332 Mailbox-related - standing at mailbox - The pedestrian was going to/from or depositing/removing items in/from a mailbox or newspaper box and was struck while standing next to the box.

339 Mailbox-related - other/unknown - The pedestrian was going to/from or depositing/removing items in/from a mailbox or newspaper box, but the specific circumstances are different from those above or are unknown.

341 Commercial-bus-related - The pedestrian was struck crossing in front of a commercial bus stopped at a marked bus stop.

342 School-bus-related - The pedestrian was struck going to or from a school bus or school bus stop.

360 Ice cream/vendor-truck-related - The pedestrian was struck going to or from an ice cream truck or other type of vehicle vending from the curb or roadside.

Walking along roadway (400)

410 Walking along roadway - with traffic, from behind - The pedestrian was walking/running along the roadway with traffic and was struck from behind.

420 Walking along roadway - with traffic, from front - The pedestrian was walking/running along the roadway with traffic and was struck from the front.

430 Walking along roadway - against traffic, from behind - The pedestrian was walking/running along the roadway against traffic and was struck from behind.

440 Walking along roadway - against traffic, from front - The pedestrian was walking/running along the roadway against traffic and was struck from the front.

490 Walking along roadway - position unknown - The pedestrian was walking/running along the roadway, but there is insufficient information to determine the position of the pedestrian at the time of the crash.

\section{Waiting to cross (500)}

510 Waiting to cross - vehicle turning - The pedestrian was standing near the curb or roadway edge and waiting to cross the roadway when they were struck by a turning vehicle.

520 Waiting to cross - vehicle not turning - The pedestrian was standing near the curb or roadway edge and waiting to cross the roadway when they were struck by a vehicle that was not turning.

590 Waiting to cross - vehicle action unknown - The pedestrian was standing near the curb or roadway edge and waiting to cross the roadway when they were struck by a vehicle, but it could not be determined if the vehicle was turning or not.

Other (600)

610 Other - standing in roadway - The pedestrian was standing in the roadway prior to the crash, but the crash cannot be further classified. 
620 Other - walking in roadway - The pedestrian was walking in the roadway prior to the crash, but the crash cannot be further classified.

680 Other - non-intersection - The crash occurred at a non-intersection location, but the actions of the pedestrian prior to the crash cannot be determined.

690 Other - intersection - The crash occurred at an intersection, but the actions of the pedestrian prior to the crash cannot be determined.

\section{Crossing/in roadway (700)}

710 Multiple threat - The pedestrian entered the traffic lane in front of standing or stopped traffic and was struck by a vehicle traveling in the same direction as the stopped traffic.

721 Right-turn - same direction - The pedestrian was traveling in the same direction as the motorist and was struck by a right-turning vehicle preparing to turn or merge, actually turning or merging, or just completing a turn or merge maneuver.

722 Right-turn - opposite direction - The pedestrian was traveling in the opposite direction as the motorist and was struck by a right-turning vehicle preparing to turn or merge, actually turning or merging, or just completing a turn or merge maneuver.

723 Left-turn - same direction - The pedestrian was traveling in the same direction as the motorist and was struck by a left-turning vehicle preparing to turn or merge, actually turning or merging, or just completing a turn or merge maneuver.

724 Left-turn - opposite direction - The pedestrian was traveling in the opposite direction as the motorist and was struck by a left-turning vehicle preparing to turn or merge, actually turning or merging, or just completing a turn or merge maneuver.

729 Turn/merge - direction unknown - The pedestrian was struck by a vehicle preparing to turn or merge, actually turning or merging, or just completing a turn or merge maneuver, but the travel directions of one or both parties is unknown.

730 Trapped - The pedestrian was struck while crossing at a signalized intersection when the light changed and traffic started moving.

741 Dash - The pedestrian ran into the roadway and was struck by a vehicle whose view of the pedestrian was not obstructed.

742 Dart-out - The pedestrian walked or ran into the roadway and was struck by a motorist whose view of the pedestrian was blocked until an instant before impact.

761 Pedestrian failed to yield - walked into vehicle - The pedestrian failed to yield to the motorist and walked into (i.e., struck) the vehicle.

762 Pedestrian failed to yield - misjudged gap - The pedestrian failed to yield to the motorist and misjudged the gap available for crossing the roadway.

763 Pedestrian failed to yield - step-out - The pedestrian failed to yield to the motorist and stepped into the travel lane and was instantaneously struck.

769 Pedestrian failed to yield - other - The pedestrian failed to yield to the motorist, but does not conform to any of the circumstances described above.

770 Motorist failed to yield - The motorist failed to yield to the pedestrian. 
Off roadway (800)

810 Off roadway - vehicle entering driveway/alley - The motor vehicle turned into a driveway/alley and struck a pedestrian on a sidewalk/walkway or driveway crossing.

820 Off roadway - vehicle exiting driveway/alley - The motor vehicle was exiting a driveway/alley and struck a pedestrian on a sidewalk/walkway or driveway crossing.

830 Off roadway - parking lot - The motor vehicle struck a pedestrian in a parkinglot.

890 Off roadway - other - The motor vehicle struck a pedestrian off the roadway, but there were other/unknown circumstances surrounding the crash.

\section{Unknown/crossing expressway (900)}

900 Other - unknown location - There is insufficient information to determine where the crash occurred.

910 Crossing an expressway - The pedestrian was crossing a limited access expressway or expressway ramp. 


\section{Cross and Fisher (1977) Bicyclist Crash Types}

\section{Class A: Bicyclist rides out from a driveway, alley, or other midblock location}

1. Cyclist fails to yield to motorist at a residential driveway or alley; pre-crash path perpendicular to roadway.

2. Cyclist fails to yield to motorist at a commercial driveway or alley; pre-crash path perpendicular to roadway.

3. Cyclist turns or merges into the path of motorist from a residential driveway or alley; precrash path parallel to roadway.

4. Cyclist fails to yield to motorist at a midblock location; entry is over curb or shoulder.

\section{Class B: Bicyclist rides out from a controlled intersection}

5. Cyclist fails to yield to motorist at an intersection controlled by a stop sign or a flashing red signal.

6. Cyclist fails to clear intersection controlled by signal before light turns green for cross traffic; motorist's view of cyclist is not obstructed.

7. Cyclist fails to clear intersection controlled by signal before light turns green for cross traffic; motorist's view of cyclist is obstructed by standing traffic.

\section{Class C: Motorist turns or drives out in front of bicyclist}

8. Motorist exiting from driveway, alley, or other midblock location fails to yield to cyclist.

9. At an intersection controlled by a stop sign or flashing red light, motorist obeys the sign but fails to yield to cyclist.

10. At an intersection controlled by a signal, motorist obeys signal but fails to yield to cyclist while making right turn on red.

11. Motorist backing from driveway fails to yield to cyclist.

12. Motorist fails to stop at an intersection controlled by a stop sign.

\section{Class D: Motorist overtakes bicyclist}

13. Motorist fails to detect bicyclist he/she is overtaking.

14. Motorist loses control of vehicle while overtaking cyclist; in some cases motorist is in uncontrolled slide or spin, but more often merely loses precise control and veers too far to right.

15. The motorist and the cyclist counteract each other's evasive action.

16. Motorist misjudges space required to pass cyclist.

17. Cyclist's path is obstructed, causing cyclist to strike obstruction or overtaking motorist.

\section{Class E: Bicyclist makes unexpected turn or swerve}

18. Cyclist turns left in front of motorist proceeding in the same direction.

19. Cyclist turns left in front of motorist approaching from straight ahead.

20. Cyclist loses control and swerves into the path of a motorist proceeding in the same direction. 
21. Cyclist riding on wrong side of street makes right turn in path of approaching motorist.

\section{Class F: Motorist makes unexpected turn}

22. Motorist makes left turn in front of cyclist proceeding in the same direction; in some cases cyclist is riding on wrong side of street.

23. Motorist makes left turn in front of cyclist approaching from straight ahead.

24. Motorist makes right turn in front of cyclist proceeding in a parallel path; cyclist either proceeding in same direction or from opposite direction (riding on wrong side of street).

\section{Class G: Other/infrequent crashes (includes types that could not meaningfully be classified into any of the other classes)}

25. Vehicles collide at uncontrolled intersection: crossing paths.

26. Vehicles collide head-on: wrong-way bicyclist.

27. Bicyclist overtaking motor vehicle.

28. Vehicles collide head-on: wrong-way motorist.

29. Parking lot, other open area: crossing paths.

30. Vehicles collide head-on: counteractive evasive action.

31. Bicyclist cuts corner when turning left: crossing paths.

32. Bicyclist swings wide when turning right: crossing paths.

33. Motorist cuts corner when turning left: crossing paths.

34. Motorist swings wide when turning right: crossing paths.

35. Motorist drive-out from on-street parking

36. Weird.

37. Insufficient information to classify. 


\section{Harkey et al. (2000) PBCAT Bicyclist Crash Types}

\section{Motorist/bicyclist turning error (110)}

111 Motorist turning error - left turn - The motorist made a left turn, cut the corner and entered the opposing traffic lane.

112 Motorist turning error - right turn - The motorist made a right turn, swung too wide and entered the opposing traffic lane.

113 Motorist turning error - other - The motorist made another type of turning error which led them into the path of the bicyclist.

114 Bicyclist turning error - left - The bicyclist made a left turn, cut the corner and entered the opposing traffic lane.

115 Bicyclist turning error - right - The bicyclist made a right turn, swung too wide and entered the opposing traffic lane.

116 Bicyclist turning error - other - The bicyclist made another type of turning error which led them into the path of the motorist.

\section{Bicyclist lost control (120)}

121 Bicyclist lost control - mechanical problems - The bicyclist lost control due to mechanical problems.

122 Bicyclist lost control - oversteering/improper braking/speed - The bicyclist lost control due to oversteering, improper braking, or speed too fast for conditions.

123 Bicyclist lost control - alcohol/drug impairment - The bicyclist lost control due to alcohol/drug impairment.

124 Bicyclist lost control - surface conditions - The bicyclist lost control due to surface conditions (sand, debris, potholes, ice, etc.).

129 Bicyclist lost control - other/unknown - The bicyclist lost control due to other/unknown circumstances.

\section{Motorist lost control (130)}

131 Motorist lost control - mechanical problems - The motorist lost control due to mechanical problems.

132 Motorist lost control - oversteering/improper braking/speed - The motorist lost control due to oversteering, improper braking, or speed too fast for conditions.

133 Motorist lost control - alcohol/drug impairment - The motorist lost control due to alcohol/drug impairment.

134 Motorist lost control - surface conditions - The motorist lost control due to surface conditions (potholes, ice, etc.).

139 Motorist lost control - other/unknown - The motorist lost control due to other/unknown circumstances. 
Sign control - intersection (140)

141 Motorist drive-out - sign control intersection - The motorist was facing the sign or flashing signal and drove into the crosswalk area or intersection and collided with the bicyclist after stopping or yielding.

142 Bicyclist drive-out - sign control intersection - The bicyclist was facing the sign or flashing signal and rode into the intersection and collided with the motorist after stopping or yielding.

143 Motorist drive-through - sign control intersection - The motorist violated the sign or flashing signal and drove into the crosswalk area or intersection and collided with the bicyclist.

144 Bicyclist drive-through - sign control intersection - The bicyclist violated the sign or flashing signal and rode into the intersection and collided with the motorist.

148 Sign control intersection - other - The crash occurred at a sign-controlled intersection but cannot be further classified.

\section{Signal control - intersection (150)}

151 Motorist drive-out - ROTR - The motorist was facing a red signal, stopped, and then drove into the crosswalk area or intersection and collided with the bicyclist while attempting to make a right turn on red.

152 Motorist drive-out - signal control intersection - The motorist was facing a red signal, stopped, and then drove into the crosswalk area or intersection and collided with the bicyclist.

153 Bicyclist ride-out - signal control intersection - The bicyclist was facing a red signal, stopped, and then rode into the intersection and collided with the motorist.

154 Motorist drive-through - signal control intersection - The motorist violated the signal and drove into the crosswalk area or intersection and collided with the bicyclist.

155 Bicyclist ride-through - signal control intersection - The bicyclist violated the signal and rode into the intersection and collided with the motorist.

156 Bicyclist failed to clear - trapped - The bicyclist lawfully entered the intersection on green but did not clear the intersection before the signal changed to green for the cross-street traffic and was struck by a vehicle whose view was not obstructed by standing or stopped traffic.

157 Bicyclist failed to clear - multiple threat - The bicyclist lawfully entered the intersection on green but did not clear the intersection before the signal changed to green for the cross-street traffic and was struck by a vehicle whose view was obstructed by standing or stopped traffic.

158 Signal control intersection - other - The crash occurred at a signal-controlled intersection but cannot be further classified.

\section{Uncontrolled intersection (160)}

160 Uncontrolled intersection - The crash occurred at an intersection not controlled by signs or signals. 
Crossing path - intersection other (180)

180 Crossing path - intersection other - The crash involved a bicyclist and motorist on initial crossing paths but cannot be further classified.

Motorist turn/merge (210)

211 Motorist left turn - same direction - The motorist turned left in front of a bicyclist going in the same direction.

212 Motorist left turn - opposite direction - The motorist turned left in front of a bicyclist coming from the opposite direction.

213 Motorist right turn - same direction - The motorist turned right in front of a bicyclist going in the same direction.

214 Motorist right turn - opposite direction - The motorist turned right in front of a bicyclist coming from the opposite direction.

215 Motorist drive-in/out - parking - The motorist struck the bicyclist while exiting or entering on-street parking.

216 Bus/delivery vehicle pullover - The bicyclist was struck by a bus or delivery vehicle pulling into or away from the curb.

Bicyclist turn/merge (220)

221 Bicyclist left turn - same direction - The bicyclist turned or swerved left in front of a motorist going in the same direction.

222 Bicyclist left turn - opposite direction - The bicyclist turned or swerved left in front of a motorist coming from the opposite direction.

223 Bicyclist right turn - same direction - The bicyclist turned or swerved right in front of a motorist going in the same direction.

224 Bicyclist right turn - opposite direction - The bicyclist turned or swerved right in front of a motorist coming from the opposite direction.

225 Bicyclist ride-out - sidewalk - The bicyclist, initially on the sidewalk, entered the roadway and subsequent path of a motor vehicle from a curb cut.

Motorist overtaking (230)

231 Motorist overtaking - undetected bicyclist - The motorist was overtaking the bicyclist and failed to detect the bicyclist.

232 Motorist overtaking - misjudged space - The motorist was overtaking the bicyclist and misjudged the width and distance required to pass the bicyclist.

239 Motorist overtaking - other/unknown - The motorist was overtaking the bicyclist but the specific circumstances surrounding the overtaking maneuver do not conform to either of the other situations described or are unknown.

Bicyclist overtaking (240)

241 Bicyclist overtaking - right side - The bicyclist struck a motor vehicle in the travel lane while passing on the right. 
242 Bicyclist overtaking - left side - The bicyclist struck a motor vehicle in the travel lane while passing on the left.

243 Bicyclist overtaking - parked vehicle - The bicyclist struck a parked vehicle while passing.

244 Bicyclist overtaking - extended door - The bicyclist struck an extended door on a parked vehicle while passing.

249 Bicyclist overtaking - other/unknown - The specific circumstances surrounding the overtaking maneuver of the bicyclist do not conform to any of the situations described or are unknown.

\section{Head-on (250)}

250 Head-on - Either operator was going the wrong way, and the two parties collided head-on.

Parallel path - other (280)

280 Parallel path - other - The crash involved a bicyclist and motorist on initial parallel paths but cannot be further classified.

\section{Bicyclist ride-out - non-intersection (310)}

311 Bicyclist ride-out - residential driveway - The bicyclist rode into the roadway or sidewalk/driveway crossing area and into the path of a motor vehicle from a residential driveway.

312 Bicyclist ride-out - commercial driveway/alley - The bicyclist rode into the roadway or sidewalk/driveway crossing area and into the path of a motor vehicle from a commercial driveway or alley.

318 Bicyclist ride-out - non-intersection other - The bicyclist rode into the roadway or sidewalk/driveway crossing area and into the path of a motor vehicle from a midblock area other than a driveway or alley.

319 Bicyclist ride-out - non-intersection unknown - The bicyclist rode into the roadway or sidewalk/driveway crossing area and into the path of a motor vehicle from an unknown midblock area.

\section{Motorist drive-out non-intersection (320)}

321 Motorist drive-out - residential driveway - The motorist drove into the roadway or sidewalk/driveway crossing area and into the path of a bicyclist from a residential driveway.

322 Motorist drive-out - commercial driveway/alley - The motorist drove into the roadway or sidewalk/driveway crossing area and into the path of a bicyclist from a commercial driveway or alley.

328 Motorist drive-out - non-intersection other - The motorist drove into the roadway or sidewalk/driveway crossing area and into the path of a bicyclist from a midblock area other than a driveway or alley. 
329 Motorist drive-out - non-intersection unknown - The motorist drove into the roadway or sidewalk/driveway crossing area and into the path of a bicyclist from an unknown midblock area.

Crossing path - non-intersection other (380)

380 Crossing path - non-intersection other - The crash involved a bicyclist and motorist on initial crossing paths but cannot be further classified.

Unusual specific circumstances

400 Bicycle only - The crash involved a bicycle but no motor vehicle.

510 Motorist intentionally caused - The motorist intentionally caused the crash.

520 Bicyclist intentionally caused - The bicyclist intentionally caused the crash.

600 Backing vehicle - The crash involved a motor vehicle that was backing and did not involve a play vehicle.

700 Play vehicle-related - The bicyclist was riding a child's vehicle such as a tricycle (not an adult tricycle), bicycle with training wheels, or "Big Wheel" type tricycle.

800 Unusual circumstances - There were other unusual circumstances not defined above (e.g., bicyclist struck by falling cargo.

910 Non-roadway - other - The crash occurred off the roadway but cannot be further classified.

990 Unknown/insufficient information - There is insufficient information to determine where the crash occurred. 


\section{REFERENCES}

Note: Numbers in parentheses that start with the letters PB are ordering numbers for items that are available for purchase from the National Technical Information Service, Springfield, VA 22161, 703-605-6000 or 800-553-NTIS. Accuracy of numbers and availability of stock should be confirmed when ordering.

\section{References in Alphabetical Order}

Applied Management Sciences, Inc. Development of a Preschool Child Pedestrian Traffic Safety Program, The Walking in Traffic Safely (WITS) Program for Preschoolers. Final Report, DOT-HS-806-678, January 1985. Washington, DC: National Highway Traffic Safety Administration. (PB 85166536)

Baker, J.S. Concepts and Classification of Traffic Accident Causes, Part II-Analysis of Accident Causes. International Road Safety and Traffic Review, 1961, 9(4), 17-24.

Baker, J.S., \& Ross, H.L. Concepts and Classification of Traffic Accident Causes, Part I. International Road Safety and Traffic Review, 1961, 9(3), 11-19.

Berger, W.G. Urban Pedestrian Accident Countermeasures Experimental Evaluation. Volume 1. Behavior Evaluation Studies. Final Report, DOT HS-801 346, February 1975. Washington, DC: National Highway Traffic Safety Administration. (PB 240255)

Berger, W.G. Urban Pedestrian Accident Countermeasures Experimental Evaluation. Volume II - Appendix A. Review of Education and Public Information Materials. Final Report, DOT HS-801 348, February 1975. Washington, DC: National Highway Traffic Safety Administration. (PB 240257)

Bittner, S.R., Chiplock, L.W., \& Dueker, R.L. Identification and Feasibility Test of Specialized Rural Pedestrian Safety Training. Vol. III - Pedsafe Junior/Senior High School Materials. Final Report, DOT HS 805 965, March 1981. Washington, DC: National Highway Traffic Safety Administration. (PB 82109646)

Blatt, J., \& Dueker, R.L. Assessment of the Safety-Relevance of Pedestrian and Bicyclist Programs. Volume One: Conduct and Results. Final Report, DOT HS-806-436, April 1983. Washington, DC: National Highway Traffic Safety Administration. (PB 83251660)

Blomberg, R.D., \& Cleven, A.M. Development, Implementation, and Evaluation of a Countermeasure Program for Alcohol-Involved Pedestrian Crashes. Final Report, DOT HS 809 067, July 2000. Washington, DC: National Highway Traffic Safety Administration. (PB 2000106890)

Blomberg, R.D., \& Cleven, A.M. Development, Implementation and Evaluation of a Pedestrian Safety Zone for Elderly Pedestrians. Final Report, DOT HS 808 692, February 1998. Washington, DC: National Highway Traffic Safety Administration. (PB 98132442) 
Blomberg, R.D., \& Cleven, A.M. Pilot Test of Heed the Speed, a Program to Reduce Speeds in Residential Neighborhoods. Final Report, DOT HS 810 648, August 2006. Washington, DC: National Highway Traffic Safety Administration.

Blomberg, R.D., \& Cleven, A.M.. Resource Guide on Laws Related to Pedestrian and Bicycle Safety. DOT HS 809 368, 2000. Washington, DC: National Highway Traffic Safety Administration.

Blomberg, R.D., Cleven, A. M., \& Edwards, J. M. Development of Safety Information Materials and Media Plans for Elderly Pedestrians. Final Report, DOT HS 808 132, June 1993. Washington, DC: National Highway Traffic Safety Administration.

Blomberg, R.D., Hale, A., \& Kearney, E.F. Development of Model Regulations for Pedestrian Safety. Final Report, DOT HS-801 287, November 1974. Washington, DC: National Highway Traffic Safety Administration. (PB 238280)

Blomberg, R.D., Hale, A., \& Preusser, D.F. Conspicuity for Pedestrians and Bicyclists: Definition of the Problem, Development and Test of Countermeasures. Final Report, DOT HS-806 563, April 1984. Washington, DC: National Highway Traffic Safety Administration. (PB 84240985)

Blomberg, R.D., Leaf, W.A., Hale, A., Farrell, M.L., \& Cross, K.D. Identification and Development of Countermeasures for Bicyclists/Motor-Vehicle Problem Types. Volume IMethods and Training Program Descriptions. Final Report DOT-HS 806 326, August 1982. Washington, DC: National Highway Traffic Safety Administration. (PB 83223917)

Blomberg, R.D., Leaf, W.A., Hale, A., Farrell, M.L., \& Cross, K.D. Identification and Development of Countermeasures for Bicyclists/Motor-Vehicle Problem Types. Volume II Public Information and Education Messages. Final Report, DOT HS-806-327, August 1982. Washington, DC: National Highway Traffic Safety Administration. (PB 83223925)

Blomberg, R.D., Leaf, W.A., Hale, A., Farrell, M.L., \& Cross, K.D. Identification and Development of Countermeasures for Bicyclists/Motor-Vehicle Problem Types. Volume III - Model Regulations. Final Report, DOT HS-806-328, August 1982. Washington, DC: National Highway Traffic Safety Administration. (PB 83223933)

Blomberg, R.D., \& Preusser, D.F. Identification and Test of Pedestrian Safety Messages for Public Education Programs. Final Report, DOT HS-801-457, March 1975. Washington, DC: National Highway Traffic Safety Administration. (PB 242010)

Blomberg, R.D., Preusser, D.F., Hale, A., \& Leaf, W.A. Experimental Field Test of Proposed Pedestrian Safety Messages. Volume I - Methods and Materials Development. Final Report, DOT HS-806-521, November 1983. Washington, DC: National Highway Traffic Safety Administration. (PB 84211572)

Blomberg, R.D., Preusser, D.F., Hale, A., \& Leaf, W.A. Experimental Field Test of Proposed Pedestrian Safety Messages. Volume II - Child Messages. Final Report, DOT 
HS-806-522, November 1983. Washington, DC: National Highway Traffic Safety Administration. (PB 84211580)

Blomberg, R.D., Preusser, D.F., Hale, A., \& Leaf, W.A. Experimental Field Test of Proposed Pedestrian Safety Messages.. Volume III - Adult Messages. Final Report, DOT HS-806-523, November 1983. Washington, DC: National Highway Traffic Safety Administration. (PB 84211598)

Blomberg, R.D., Preusser, D.F., Hale, A., \& Ulmer, R.G. A Comparison of Alcohol Involvement in Pedestrians and Pedestrian Casualties. Final Report, DOT HS-805-249, October 1979. Washington, DC: National Highway Traffic Safety Administration. (PB $80166275)$

Brown, R., \& Sutherland, L.C. An Audible Automobile Back-up Pedestrian Warning Device - Development and Evaluation. Final Report, DOT HS-802 083, November 1976. Washington, DC: National Highway Traffic Safety Administration. (PB 262806)

Casey, S.M., Cross, K.D., Leaf, W.A., \& Blomberg, R.D. Bicyclists' Inclination and Ability to Search Behind Before Turning Left. Interim Report, DOT HS-805 893, February 1980. Washington, DC: National Highway Traffic Safety Administration. (PB 81216608)

Chiplock, L.W., \& Dueker, R.L. Identification and Feasibility Test of Specialized Rural Pedestrian Safety Training. Vol. II - Pedsafe Elementary Materials. Final Report, DOT HS 805 964, March 1981. Washington, DC: National Highway Traffic Safety Administration. (PB 82109638)

Chiplock, L.W., Dueker, R.L., \& Bittner, S.R. Identification and Feasibility Test of Specialized Rural Pedestrian Safety Training. Vol. IV-Pedsafe Audiovisual Scripts. Final Report, DOT HS 805 966, March 1981. Washington, DC: National Highway Traffic Safety Administration. (PB 82109653)

Cleven, A.M., \& Blomberg, R.D. Development and Evaluation of a Pedestrian Safety Training Program for Elementary School Bus Riders. Final Report, DOT HS 808267 , December 1994.

Compton, R.P., \& Milton, E.V. Safety Impact of Permitting Right-Turn-on-Red: A Report to Congress by the National Highway Traffic Safety Administration. Report to Congress, DOT HS 808 200, December 1994. Washington, DC: National Highway Traffic Safety Administration. (PB 95197463)

Cross, K.D., \& Fisher, G. A Study of Bicycle/Motor-Vehicle Accidents: Identification of Problem Types and Countermeasure Approaches. Volume 1. Final Report, DOT-HS-803 315, September 1977. Washington, DC: National Highway Traffic Safety Administration. (PB 282280)

Cross, K.D., \& Fisher, G. A Study of Bicycle/Motor-Vehicle Accidents: Identification of Problem Types and Countermeasure Approaches.. Volume 2 - Appendices A-D. Final 
Report, DOT HS 803 316, September 1977. Washington, DC: National Highway Traffic Safety Administration. (PB 282281)

Cross, K.D., \& Fisher, G. A Study of Bicycle/Motor-Vehicle Accidents: Identification of Problem Types and Countermeasure Approaches. Volume 3 - Coding Index - Appendices $E-G$. Final Report, DOT HS 803 317, September 1977. Washington, DC: National Highway Traffic Safety Administration. (PB 282572)

DeBartolo, K.B., Preusser, D.F., \& Blomberg, R.D. Enforcement Frequency, Sanctions and Compliance Level for Pedestrian Safety. Final Report, DOT HS-803 650, April 1978. Washington, DC: National Highway Traffic Safety Administration. (PB 288792)

Dueker, R.L. Experimental Field Test of Proposed Anti-Dart-Out Training Programs. Volume 1: Conduct and Results. Final Report, DOT HS-806-195, December 1981. Washington, DC: National Highway Traffic Safety Administration. (PB 83112656)

Dueker, R.L. Threat Detection Training Programs for Child Pedestrian Safety. Volume I: Conduct, Results, and Recommendations. Final Report, DOT HS-801 450, March 1975. Washington, DC: National Highway Traffic Safety Administration. (PB 241181)

Dueker, R.L., \& Berger, S.S. Experimental Field Test of Proposed Anti-Dart-Out Training Programs. Volume 2 - Implementation Guidelines and Program Materials. Final Report, DOT HS-806-196, December 1981. Washington, DC: National Highway Traffic Safety Administration. (PB 83112664)

Dueker, R.L., \& Berger, S.S. Experimental Field Test of Proposed Anti-Dart-Out Training Programs. Volume 3 - Program Staff Training Materials and Videotape/Film. Final Report, DOT HS-806-197, December 1981. Washington, DC: National Highway Traffic Safety Administration. (PB 84158419)

Dueker, R.L., \& Chiplock, L.W. Identification and Feasibility Test of Specialized Rural Pedestrian Safety Training. Volume. 1 - Program Development and Training. Final Report, DOT HS 806 256, March 1981. Washington, DC: National Highway Traffic Safety Administration. (PB 85155653)

Dunlap and Associates, Inc. Bicycle and Pedestrian Safety Resource Guide, DOT HS 809 977, 2006. Available for download at: http://www.nhtsa.dot.gov/people/injury/pedbimot/bike/BikePedestrian/index.htm. Washington, DC: National Highway Traffic Safety Administration.

Dunlap and Associates, Inc. Bicycle Safety Resource Guide, 1998.

Ehrlich, P., Farina, A., Pavlinski, L., \& Tarrants, W.E. Effectiveness and Efficiencies in Pedestrian Safety. Evaluation Summary Report 1966-1981, DOT HS-806 131, March 82. Washington, DC: National Highway Traffic Safety Administration. (PB 83110429) 
Fell, J.C. A Motor-Vehicle Accident Causal System: The Human Element. DOT HS-801214, 1974. Washington, DC: National Highway Traffic Safety Administration. (PB 235918)

Fell, J.C., \& Toth, G.R. Pedestrian Accidents: A State-of-the-Art 1970-1980. Technical Report, DOT HS 806 270, September 1981. Washington, DC: National Highway Traffic Safety Administration. (PB 83157511)

Hale, A., Blomberg, R.D., \& Kearney, E.F. Model Regulations and Public Education for Rural-Suburban Pedestrian Safety. Final Report, DOT HS-805 639, August 1980. Washington, DC: National Highway Traffic Safety Administration. (PB 81137762)

Hale, A., Blomberg, R.D., \& Preusser, D.F. Experimental Field Test of the Model Ice Cream Truck Ordinance in Detroit. Final Report, DOT HS-803 410, April 1978. Washington, DC: National Highway Traffic Safety Administration. (PB 283419)

Hale, A., Shapiro, R.G., Blomberg, R.D., \& Kearney, E.F. Development and Test of Rural Pedestrian Safety Countermeasures. Final Report, DOT HS-806-518, December 1983. Washington, DC: National Highway Traffic Safety Administration. (PB 84186410)

Hale, A., \& Zeidler, P. Review of the Literature and Programs for Pedestrian and Bicyclist Conspicuity. Final Report, DOT HS-806 564, April 1984. Washington, DC: National Highway Traffic Safety Administration. (PB 85161511)

Hanowski, R.J., Spaulding, J.M., Gaskins, C., Schaudt, W.A., Miller, S., Holbrook, T., Olson, R.L., Dingus, T.A., Hickman, J.S., Huey, R., \& Llaneras, E.E. Field Evaluation of Alternative Automated Systems for Reducing Illegal Passing of School Buses. Final Report, awaiting release from NHTSA. Washington, DC: National Highway Traffic Safety Administration.

Harkey, D.L., Mekemson, J., Chen, M., \& Krull, K.A. Pedestrian and Bicycle Crash Analysis Tool (PBCAT) Software and User's Manual. FHWA-RD-99-192, June 2000. Washington, DC: Federal Highway Administration.

Hunter, W. W., Pein, W. E., \& Stutts, J. C. Bicycle Crash Types: A 1990s Informational Guide, Research Report, Contract No. DTFH61-92-Y-30048, December 1995 . Washington, DC: Federal Highway Administration

Hunter, W.W., Stutts, J.C., Pein, W.E., \& Cox, C.L. Pedestrian and Bicycle Crash Types of the Early 1990s. Publication No. FHWA-RD-95-163, 1995. Washington, DC: Federal Highway Administration.

Kane, J.N. Famous First Facts. H. W. Wilson Co., New York, 4th Ed., 1981.

Knoblauch, R.L. Causative Factors and Countermeasures for Rural and Suburban Pedestrian Accidents: Accident Data Collection and Analysis. Final Report, DOT-HS-802 266, March 1977. Washington, DC: National Highway Traffic Safety Administration. (PB 265162) 
Knoblauch, R.L. Urban Pedestrian Accident Countermeasures Experimental Evaluation. Volume II. Accident Studies. Final Report, DOT HS-801 347, February 1975. Washington, DC: National Highway Traffic Safety Administration. (PB 240256)

Knoblauch, R.L., Moore, Jr., W., Schmitz, P.R., and Sommers, B.J. Causative Factors and Countermeasures for Rural and Suburban Pedestrian Accidents: Accident Data Collection and Analysis - Appendices. Final Report, DOT-HS-802 474, June 1977. Washington, DC: National Highway Traffic Safety Administration. (PB 270107)

Leaf, W.A., \& Blomberg, R.D. Development and Test of Selected Model Pedestrian Safety Regulations. Final Report, DOT HS-805 901, April 1981. Washington, DC: National Highway Traffic Safety Administration. (PB 81219297)

Leaf, W.A., \& Preusser, D.F. Identification of Alcohol-Pedestrian Crash Problems Among Culturally Diverse Groups. Final Report, DOT HS 808 641, 1997. Washington, DC:

National Highway Traffic Safety Administration. (PB 98110653)

Leaf, W.A., \& Preusser, D.F. Literature Review on Vehicle Travel Speeds and Pedestrian Injuries. Final Report, DOT HS 809 021, October 1999. Washington, DC: National Highway Traffic Safety Administration. (PB 94109568)

McGlade, F., \& Laws, F.D. Classifying Accidents: A Theoretical Viewpoint. Traffic Safety Research Review, 1962, 6(1), 2-9.

National Highway Traffic Safety Administration. Pedestrian Injury Reduction Research. Report to the Congress, DOT HS 808 026, June 1993. Washington, DC: National Highway Traffic Safety Administration. (PB 94109568)

National Highway Traffic Safety Administration. Compendium of Traffic Safety Projects: A Decade and Beyond. Annotated Bibliography, DOT HS 808 379, April 1996. Washington, DC: National Highway Traffic Safety Administration. (PB 96178330)

National Highway Traffic Safety Administration. Manual Accident Typing for Pedestrian Accidents: Coder's Handbook. DOT HS 806 354, January 1983. Washington, DC: National Highway Traffic Safety Administration.

National Highway Traffic Safety Administration. Traffic Safety Facts, 2004 DataPedalcyclists, DOT HS 809 912. Washington, DC: National Highway Traffic Safety Administration.

National Highway Traffic Safety Administration. Traffic Safety Facts, 2004 DataPedestrians, DOT HS 809 913. Washington, DC: National Highway Traffic Safety Administration.

National Highway Traffic Safety Administration. Walk Alert - A National Pedestrian Safety Program Guide. 1994. Washington, DC: National Highway Traffic Safety Administration. 
Partnership for a Walkable America. Contract No. DTFH61-92-C-00138. Washington, DC: Federal Highway Administration.

Perchonok, K. Accident Cause Analysis. NHTSA, DOT HS-053-1-109, 1975. Washington, DC: National Highway Traffic Safety Administration.

Phinney, J., Colker, L., \& Cosgrove, M. Literature Review on the Preschool Pedestrian. Final Report, DOT HS-806-679, January 1985. Washington, DC: National Highway Traffic Safety Administration. (PB 85170009)

Preusser, D.F., Blomberg, R.D., Edwards, J.M., Farrell, M.L., \& Preusser, C.W. The Development and Test of Urban and Rural Pedestrian Safety Messages. Final Report, DOT-HS-806-682, January 1985. Washington, DC: National Highway Traffic Safety Administration. (PB 85160711)

Preusser, D.F., Leaf, W.A., DeBartolo, K.B., \& Blomberg, R.D. The Effect of Right-Turnon-Red on Pedestrian and Bicyclist Accidents. Final Report, DOT-HS-806-182, October 1981. Washington, DC: National Highway Traffic Safety Administration. (PB 82238445)

Preusser, D.F., \& Lund, A.K. And Keep on Looking: A Film to Reduce Pedestrian Crashes Among 9- to 12-Year-Olds. Journal of Safety Research, (1988), 19(4), 177-195.

Rose, A.M., Levine, J.M., \& Eisner, E.J. Measurement of Pedestrian Behavior. Final Report, DOT HS-802 105, November 1976. Washington, DC: National Highway Traffic Safety Administration. (PB 262647)

Singer, S. Pedestrian Regulation Enforcement and the Incidence of Pedestrian Accidents. Final Report, SSD-69-726, August 1969. Washington, DC: National Highway Traffic Safety Administration. (PB 187519)

Snyder, M.B., \& Knoblauch, R.L. Pedestrian Safety: The Identification of Precipitating Factors and Possible Countermeasures, Volume I. Final Report, DOT HS-800 403, January 1971. Washington, DC: National Highway Traffic Safety Administration. (PB 197749)

Snyder, M.B., \& Knoblauch, R.L. Pedestrian Safety: The Identification of Precipitating Factors and Possible Countermeasures, Volume II Appendices. Final Report, DOT HS800 404, January 1971. Washington, DC: National Highway Traffic Safety Administration. (PB 197750)

Stutts, J.C., Hunter, W.W., Tracy, L., \& Wilkinson,W.C., III. Pedestrian and Bicyclist Safety: A Review of Key Program and Countermeasure Development During the 1980s. Final Report, DOT HS 808 108, March 1992.

Thackray, Jr., R.M., \& Dueker, R.L. Child Pedestrian Supervision/Guidance. Final Report, DOT-HS-806-519, January 1983. Washington, DC: National Highway Traffic Safety

Administration. (PB 84186436) 
Tracy, L. Procedures and Resource Guide for Bicycle Helmet Promotions: A Review of Bicycle Helmet Promotions in the United States. Final Report, DOT HS 807 963, September 1992. Washington, DC: National Highway Traffic Safety Administration. (PB 93184307)

Ulmer, R.G., Leaf, W.A., \& Blomberg, R.D. Analysis of the Dismounted Motorist and Road-Worker Model Pedestrian Safety Regulations. Final Report, DOT HS-806-445, August 1982. Washington, DC: National Highway Traffic Safety Administration. (PB $84121250)$

Vayda, A., \& Crespi, I. Public Acceptability of Highway Safety Countermeasures. Volume IV: Pedestrian Safety. Final Report, DOT HS-805-973, June 1981. Washington, DC:

National Highway Traffic Safety Administration. (PB 82110446)

Zylman, R., Blomberg, R.D., \& Preusser, D.F. A Review of the Literature on the Involvement of Alcohol in Pedestrian Collisions Resulting in Death and Injury. Interim Report, DOT HS- 801 413, February 1973. Washington, DC: National Highway Traffic Safety Administration. (PB 240640)

\section{References by Ascending Date of Publication}

Baker, J.S., \& Ross, H.L. Concepts and Classification of Traffic Accident Causes, Part I. International Road Safety and Traffic Review, 1961, 9(3), 11-19.

Baker, J.S. Concepts and Classification of Traffic Accident Causes, Part II-Analysis of Accident Causes. International Road Safety and Traffic Review, 1961, 9(4), 17-24.

McGlade, F., \& Laws, F.D. Classifying Accidents: A Theoretical Viewpoint. Traffic Safety Research Review, 1962, 6(1), 2-9.

Singer, S. Pedestrian Regulation Enforcement and the Incidence of Pedestrian Accidents. Final Report, SSD-69-726, . Washington, DC: National Highway Traffic Safety Administration. (PB 187519)

Snyder, M.B., \& Knoblauch, R.L. Pedestrian Safety: The Identification of Precipitating Factors and Possible Countermeasures, Volume I. Final Report, DOT HS-800 403, January 1971. Washington, DC: National Highway Traffic Safety Administration. (PB 197749)

Snyder, M.B., \& Knoblauch, R.L. Pedestrian Safety: The Identification of Precipitating Factors and Possible Countermeasures, Volume II Appendices. Final Report, DOT HS800 404, January 1971. Washington, DC: National Highway Traffic Safety Administration. (PB 197750)

Zylman, R., Blomberg, R.D., \& Preusser, D.F. A Review of the Literature on the Involvement of Alcohol in Pedestrian Collisions Resulting in Death and Injury. Interim Report, DOT HS- 801 413, February 1973. Washington, DC: National Highway Traffic Safety Administration. (PB 240640) 
Blomberg, R.D., Hale, A., \& Kearney, E.F. Development of Model Regulations for Pedestrian Safety. Final Report, DOT HS-801 287, November 1974. Washington, DC: National Highway Traffic Safety Administration. (PB 238280)

Fell, J.C. A Motor-Vehicle Accident Causal System: The Human Element. NHTSA, DOT HS-801-214, 1974. Washington, DC: National Highway Traffic Safety Administration. (PB 235918)

Berger, W.G. Urban Pedestrian Accident Countermeasures Experimental Evaluation. Volume 1. Behavior Evaluation Studies. Final Report, DOT HS-801 346, February 1975. Washington, DC: National Highway Traffic Safety Administration. (PB 240255)

Berger, W.G. Urban Pedestrian Accident Countermeasures Experimental Evaluation. Volume II - Appendix A. Review of Education and Public Information Materials. Final Report, DOT HS-801 348, February 1975. Washington, DC: National Highway Traffic Safety Administration. (PB 240257)

Knoblauch, R.L. Urban Pedestrian Accident Countermeasures Experimental Evaluation. Volume II. Accident Studies. Final Report, DOT HS-801 347, February 1975. Washington, DC: National Highway Traffic Safety Administration. (PB 240256)

Blomberg, R.D., \& Preusser, D.F. Identification and Test of Pedestrian Safety Messages for Public Education Programs. Final Report, DOT HS-801-457, March 1975. Washington, DC: National Highway Traffic Safety Administration. (PB 242010)

Dueker, R.L. Threat Detection Training Programs for Child Pedestrian Safety. Volume I: Conduct, Results, and Recommendations. Final Report, DOT HS-801 450, March 1975. Washington, DC: National Highway Traffic Safety Administration. (PB 241181)

Perchonok, K. Accident Cause Analysis. NHTSA, DOT HS-053-1-109, 1975. Washington, DC: National Highway Traffic Safety Administration.

Brown, R., \& Sutherland, L.C. An Audible Automobile Back-up Pedestrian Warning Device - Development and Evaluation. Final Report, DOT HS-802 083, November 1976. Washington, DC: National Highway Traffic Safety Administration. (PB 262806)

Rose, A.M., Levine, J.M., \& Eisner, E.J. Measurement of Pedestrian Behavior. Final Report, DOT HS-802 105, November 1976. Washington, DC: National Highway Traffic Safety Administration. (PB 262647)

Knoblauch, R.L. Causative Factors and Countermeasures for Rural and Suburban Pedestrian Accidents: Accident Data Collection and Analysis. Final Report, DOT-HS802 266, March 1977. Washington, DC: National Highway Traffic Safety Administration. (PB 265162)

Knoblauch, R.L., Moore, Jr., W., Schmitz, P.R., \& Sommers, B.J. Causative Factors and Countermeasures for Rural and Suburban Pedestrian Accidents: Accident Data 
Collection and Analysis - Appendices. Final Report, DOT-HS-802 474, June 1977.

Washington, DC: National Highway Traffic Safety Administration. (PB 270107)

Cross, K.D., \& Fisher, G. A Study of Bicycle/Motor-Vehicle Accidents: Identification of Problem Types and Countermeasure Approaches. Volume 1. Final Report, DOT-HS-803 315, September 1977. Washington, DC: National Highway Traffic Safety Administration. (PB 282280)

Cross, K.D., \& Fisher, G. A Study of Bicycle/Motor-Vehicle Accidents: Identification of Problem Types and Countermeasure Approaches.. Volume 2 - Appendices A-D. Final Report, DOT HS 803 316, September 1977. Washington, DC: National Highway Traffic Safety Administration. (PB 282281)

Cross, K.D., \& Fisher, G. A Study of Bicycle/Motor-Vehicle Accidents: Identification of Problem Types and Countermeasure Approaches. Volume 3 - Coding Index - Appendices E-G. Final Report, DOT HS 803 317, September 1977. Washington, DC: National Highway Traffic Safety Administration. (PB 282572)

DeBartolo, K.B., Preusser, D.F., \& Blomberg, R.D. Enforcement Frequency, Sanctions and Compliance Level for Pedestrian Safety. Final Report, DOT HS-803 650, April 1978. Washington, DC: National Highway Traffic Safety Administration. (PB 288792)

Hale, A., Blomberg, R.D., \& Preusser, D.F. Experimental Field Test of the Model Ice Cream Truck Ordinance in Detroit. Final Report, DOT HS-803 410, April 1978. Washington, DC: National Highway Traffic Safety Administration. (PB 283419)

Blomberg, R.D., Preusser, D.F., Hale, A., \& Ulmer, R.G. A Comparison of Alcohol Involvement in Pedestrians and Pedestrian Casualties. Final Report, DOT HS-805-249, October 1979. Washington, DC: National Highway Traffic Safety Administration. (PB 80166275)

Casey, S.M., Cross, K.D., Leaf, W.A., \& Blomberg, R.D. Bicyclists' Inclination and Ability to Search Behind Before Turning Left. Interim Report, DOT HS-805 893, February 1980. Washington, DC: National Highway Traffic Safety Administration. (PB $81216608)$

Hale, A., Blomberg, R.D., \& Kearney, E.F. Model Regulations and Public Education for Rural-Suburban Pedestrian Safety. Final Report, DOT HS-805 639, August 1980. Washington, DC: National Highway Traffic Safety Administration. (PB 81137762)

Dueker, R.L., \& Chiplock, L.W. Identification and Feasibility Test of Specialized Rural Pedestrian Safety Training. Volume. 1 - Program Development and Training. Final Report, DOT HS 806 256, March 1981. Washington, DC: National Highway Traffic Safety Administration. (PB 85155653)

Chiplock, L.W., \& Dueker, R.L. Identification and Feasibility Test of Specialized Rural Pedestrian Safety Training. Vol. II - Pedsafe Elementary Materials. Final Report, DOT 
HS 805 964, March 1981. Washington, DC: National Highway Traffic Safety Administration. (PB 82109638)

Bittner, S.R., Chiplock, L.W., \& Dueker, R.L. Identification and Feasibility Test of Specialized Rural Pedestrian Safety Training. Vol. III - Pedsafe Junior/Senior High School Materials. Final Report, DOT HS 805 965, March 1981. Washington, DC:

National Highway Traffic Safety Administration. (PB 82109646)

Chiplock, L.W., Dueker, R.L., \& Bittner, S.R. Identification and Feasibility Test of Specialized Rural Pedestrian Safety Training. Vol. IV - Pedsafe Audiovisual Scripts. Final Report, DOT HS 805 966, March 1981. Washington, DC: National Highway Traffic Safety Administration. (PB 82109653)

Fell, J.C., \& Toth, G.R. Pedestrian Accidents: A State-of-the-Art 1970-1980. Technical Report, DOT HS 806 270, September 1981. Washington, DC: National Highway Traffic Safety Administration. (PB 83157511)

Dueker, R.L. Experimental Field Test of Proposed Anti-Dart-Out Training Programs. Volume 1: Conduct and Results. Final Report, DOT HS-806-195, December 1981. Washington, DC: National Highway Traffic Safety Administration. (PB 83112656)

Dueker, R.L., \& Berger, S.S. Experimental Field Test of Proposed Anti-Dart-Out Training Programs. Volume 2 - Implementation Guidelines and Program Materials. Final Report, DOT HS-806-196, December 1981. Washington, DC: National Highway Traffic Safety Administration. (PB 83112664)

Dueker, R.L., \& Berger, S.S. Experimental Field Test of Proposed Anti-Dart-Out Training Programs. Volume 3 - Program Staff Training Materials and Videotape/Film. Final Report, DOT HS-806-197, December 1981. Washington, DC: National Highway Traffic Safety Administration. (PB 84158419)

Kane, J.N. Famous First Facts. H. W. Wilson Co., New York, 4th Ed., 1981.

Leaf, W.A., \& Blomberg, R.D. Development and Test of Selected Model Pedestrian Safety Regulations. Final Report, DOT HS-805 901, April 1981. Washington, DC: National Highway Traffic Safety Administration. (PB 81219297)

Vayda, A., \& Crespi, I. Public Acceptability of Highway Safety Countermeasures. Volume IV: Pedestrian Safety. Final Report, DOT HS-805-973, June 1981. Washington, DC: National Highway Traffic Safety Administration. (PB 82110446)

Preusser, D.F., Leaf, W.A., DeBartolo, K.B., \& Blomberg, R.D. The Effect of RightTurn-on-Red on Pedestrian and Bicyclist Accidents. Final Report, DOT-HS-806-182, October 1981. Washington, DC: National Highway Traffic Safety Administration. (PB $82238445)$ 
Ehrlich, P., Farina, A., Pavlinski, L., \& Tarrants, W.E. Effectiveness and Efficiencies in Pedestrian Safety. Evaluation Summary Report 1966-1981, DOT HS-806 131, March 1982. Washington, DC: National Highway Traffic Safety Administration. (PB 83110429)

Blomberg, R.D., Leaf, W.A., Hale, A., Farrell, M.L., \& Cross, K.D. Identification and Development of Countermeasures for Bicyclists/Motor-Vehicle Problem Types. Volume I - Methods and Training Program Descriptions. Final Report DOT-HS 806 326, August 1982. Washington, DC: National Highway Traffic Safety Administration. (PB 83223917)

Blomberg, R.D., Leaf, W.A., Hale, A., Farrell, M.L., \& Cross, K.D. Identification and Development of Countermeasures for Bicyclists/Motor-Vehicle Problem Types. Volume II - Public Information and Education Messages. Final Report, DOT HS-806-327, August 1982. Washington, DC: National Highway Traffic Safety Administration. (PB 83223925)

Blomberg, R.D., Leaf, W.A., Hale, A., Farrell, M.L., \& Cross, K.D. Identification and Development of Countermeasures for Bicyclists/Motor-Vehicle Problem Types. Volume III - Model Regulations. Final Report, DOT HS-806-328, August 1982. Washington, DC: National Highway Traffic Safety Administration. (PB 83223933)

Ulmer, R.G., Leaf, W.A., \& Blomberg, R.D. Analysis of the Dismounted Motorist and Road-Worker Model Pedestrian Safety Regulations. Final Report, DOT HS-806-445, August 1982. Washington, DC: National Highway Traffic Safety Administration. (PB $84121250)$

National Highway Traffic Safety Administration. Manual Accident Typing for Pedestrian Accidents: Coder's Handbook. DOT HS 806 354, January 1983. Washington, DC: National Highway Traffic Safety Administration.

Thackray, Jr., R.M., \& Dueker, R.L. Child Pedestrian Supervision/Guidance. Final Report, DOT-HS-806-519, January 1983. Washington, DC: National Highway Traffic Safety Administration. (PB 84186436)

Blatt, J., \& Dueker, R.L. Assessment of the Safety-Relevance of Pedestrian and Bicyclist Programs. Volume One: Conduct and Results. Final Report, DOT HS-806-436, April 1983. Washington, DC: National Highway Traffic Safety Administration. (PB 83251660)

Blomberg, R.D., Preusser, D.F., Hale, A., \& Leaf, W.A. Experimental Field Test of Proposed Pedestrian Safety Messages. Volume I - Methods and Materials Development. Final Report, DOT HS-806-521, November 1983. Washington, DC: National Highway Traffic Safety Administration. (PB 84211572)

Blomberg, R.D., Preusser, D.F., Hale, A., \& Leaf, W.A. Experimental Field Test of Proposed Pedestrian Safety Messages. Volume II - Child Messages. Final Report, DOT HS-806-522, November 1983. Washington, DC: National Highway Traffic Safety Administration. (PB 84211580)

Blomberg, R.D., Preusser, D.F., Hale, A., \& Leaf, W.A. Experimental Field Test of Proposed Pedestrian Safety Messages.. Volume III - Adult Messages. Final Report, DOT 
HS-806-523, November 1983. Washington, DC: National Highway Traffic Safety Administration. (PB 84211598)

Hale, A., Shapiro, R.G., Blomberg, R.D., \& Kearney, E.F. Development and Test of Rural Pedestrian Safety Countermeasures. Final Report, DOT HS-806-518, December 1983. Washington, DC: National Highway Traffic Safety Administration. (PB 84186410)

Blomberg, R.D., Hale, A., and Preusser, D.F. Conspicuity for Pedestrians and Bicyclists: Definition of the Problem, Development and Test of Countermeasures. Final Report, DOT HS-806 563, April 1984. Washington, DC: National Highway Traffic Safety Administration. (PB 84240985)

Hale, A., \& Zeidler, P. Review of the Literature and Programs for Pedestrian and Bicyclist Conspicuity. Final Report, DOT HS-806 564, April 1984. Washington, DC: National Highway Traffic Safety Administration. (PB 85161511)

Applied Management Sciences, Inc. Development of a Preschool Child Pedestrian Traffic Safety Program, The Walking in Traffic Safely (WITS) Program for Preschoolers. Final Report, DOT-HS-806-678, January 1985. Washington, DC: National Highway Traffic Safety Administration. (PB 85166536)

Phinney, J., Colker, L., \& Cosgrove, M. Literature Review on the Preschool Pedestrian. Final Report, DOT HS-806-679, January 1985. Washington, DC: National Highway Traffic Safety Administration. (PB 85170009)

Preusser, D.F., Blomberg, R.D., Edwards, J.M., Farrell, M.L., \& Preusser, C.W. The Development and Test of Urban and Rural Pedestrian Safety Messages. Final Report, DOT-HS-806-682, January 1985. Washington, DC: National Highway Traffic Safety Administration. (PB 85160711)

Preusser, D.F., \& Lund, A.K. And Keep on Looking: A Film to Reduce Pedestrian Crashes Among 9- to 12-Year-Olds. Journal of Safety Research, (1988), 19(4), 177-195.

Stutts, J.C., Hunter, W.W., Tracy, L., \& Wilkinson,W.C., III. Pedestrian and Bicyclist Safety: A Review of Key Program and Countermeasure Development During the 1980's. Final Report, DOT HS 808 108, March 1992. Washington, DC: National Highway Traffic Safety Administration.

Tracy, L. Procedures and Resource Guide for Bicycle Helmet Promotions: A Review of Bicycle Helmet Promotions in the United States. Final Report, DOT HS 807 963, September 1992. Washington, DC: National Highway Traffic Safety Administration. (PB 93184307)

Blomberg, R.D., Cleven, A. M., \& Edwards, J. M. Development of Safety Information Materials and Media Plans for Elderly Pedestrians. Final Report, DOT HS 808 132, June 1993. Washington, DC: National Highway Traffic Safety Administration. 
National Highway Traffic Safety Administration. Pedestrian Injury Reduction Research. Report to the Congress, DOT HS 808 026, June 1993. Washington, DC: National Highway Traffic Safety Administration. (PB 94109568)

Cleven, A.M., \& Blomberg, R.D. Development and Evaluation of a Pedestrian Safety Training Program for Elementary School Bus Riders. Final Report, DOT HS 808 267, December 1994. Washington, DC: National Highway Traffic Safety Administration.

Compton, R.P., \& Milton, E.V. Safety Impact of Permitting Right-Turn-on-Red: A Report to Congress by the National Highway Traffic Safety Administration. Report to Congress, DOT HS 808 200, December 1994. Washington, DC: National Highway Traffic Safety Administration. (PB 95197463)

National Highway Traffic Safety Administration. Walk Alert - A National Pedestrian Safety Program Guide. 1994. Washington, DC: National Highway Traffic Safety Administration.

Hunter, W. W., Pein, W. E.., \& Stutts, J. C. Bicycle Crash Types: A 1990's Informational Guide, Research Report, Contract No. DTFH61-92-Y-30048, December 1995.

Washington, DC: Federal Highway Administration.

Hunter, W.W., Stutts, J.C., Pein, W.E., \& Cox, C.L. Pedestrian and Bicycle Crash Types of the Early 1990's. Publication No. FHWA-RD-95-163, 1995. Washington, DC: Federal Highway Administration.

National Highway Traffic Safety Administration. Compendium of Traffic Safety Projects: A Decade and Beyond. Annotated Bibliography, DOT HS 808 379, April 1996. Washington, DC: National Highway Traffic Safety Administration. (PB 96178330)

Leaf, W.A., \& Preusser, D.F. Identification of Alcohol-Pedestrian Crash Problems Among Culturally Diverse Groups. Final Report, DOT HS 808 641, 1997. Washington, DC: National Highway Traffic Safety Administration. (PB 98110653)

Blomberg, R.D., \& Cleven, A.M. Development, Implementation and Evaluation of a Pedestrian Safety Zone for Elderly Pedestrians. Final Report, DOT HS 808 692, February 1998. Washington, DC: National Highway Traffic Safety Administration. (PB 98132442)

Dunlap and Associates, Inc. Bicycle Safety Resource Guide, 1998. Washington, DC: National Highway Traffic Safety Administration and Federal Highway Administration.

Leaf, W.A., \& Preusser, D.F. Literature Review on Vehicle Travel Speeds and Pedestrian Injuries. Final Report, DOT HS 809 021, October 1999. Washington, DC: National Highway Traffic Safety Administration. (PB 94109568)

Harkey, D.L., Mekemson, J., Chen, M., \& Krull, K.A. Pedestrian and Bicycle Crash Analysis Tool (PBCAT) Software and User's Manual. FHWA-RD-99-192, June 2000. Washington, DC: Federal Highway Administration. 
Blomberg, R.D., \& Cleven, A.M. Development, Implementation, and Evaluation of a Countermeasure Program for Alcohol-Involved Pedestrian Crashes. Final Report, DOT HS 809 067, July 2000. Washington, DC: National Highway Traffic Safety Administration. (PB 2000106890)

Blomberg, R.D., \& Cleven, A.M.. Resource Guide on Laws Related to Pedestrian and Bicycle Safety. DOT HS 809 368, 2000. Washington, DC: National Highway Traffic Safety Administration.

National Highway Traffic Safety Administration. Traffic Safety Facts, 2004 DataPedalcyclists, DOT HS 809 912. Washington, DC: National Highway Traffic Safety Administration.

National Highway Traffic Safety Administration. Traffic Safety Facts, 2004 DataPedestrians, DOT HS 809 913. Washington, DC: National Highway Traffic Safety Administration.

Blomberg, R.D., \& Cleven, A.M. Pilot Test of Heed the Speed, a Program to Reduce Speeds in Residential Neighborhoods. Final Report, DOT HS 810 648, August 2006. Washington, DC: National Highway Traffic Safety Administration.

Dunlap and Associates, Inc. Bicycle and Pedestrian Safety Resource Guide, DOT HS 809 977, 2006. Available for download at:

http://www.nhtsa.dot.gov/people/injury/pedbimot/bike/BikePedestrian/index.htm. Washington, DC: National Highway Traffic Safety Administration.

Hanowski, R.J., Spaulding, J.M., Gaskins, C., Schaudt, W.A., Miller, S., Holbrook, T., Olson, R.L., Dingus, T.A., Hickman, J.S., Huey, R., \& Llaneras, E.E. Field Evaluation of Alternative Automated Systems for Reducing Illegal Passing of School Buses. Final Report, awaiting release from NHTSA. Washington, DC: National Highway Traffic Safety Administration. 


\section{INDEX}

AAA, 25, 49, 109

Adults, 14, 19, 22, 27, 58, 71, 72, 73, 78, 90, 92, 97, 99, 109, 126, 128, 137

Alcohol, 7, 13, 14, 23, 27, 35, 38, 51, 56, 58, $65,89,90,91,92,122,127,128,131$, 133, 135, 138, 139

American Automobile Association, 25, 49, 109

Anticipatory phase, 11

Applied Management Sciences, 20, 96, 97, 99, 127, 137

Awareness, 26, 27, 35, 62

BAC, 14, 51, 90, 92

Baker, J.S., 9, 127, 133

Behavior measurement, 15

Behavioral approach, 5, 9

Berger, S.S., 100, 102, 103, 130, 136

Berger, W.G., 15, 21, 67, 88, 98, 100, 102, 103, 127, 130, 134, 136

Bicycle crashes, 19, 35, 40, 42, 46, 50, 53, 57, 68, 75, 83, 131, 138

Bicycle safety, 18, 26, 27, 42, 45, 51, 52, 53, $83,128,130,138,139$

Bicyclist crash type

Bicyclist drive-out, 123

Bicyclist drive-through, 123

Bicyclist intentionally caused, 126

Bicyclist lost control, 122

Bicyclist overtaking, 121, 124, 125

Bicyclist ride-out, 12, 120, 123, 124, 125

Bicyclist turn/merge, 124

Bicyclist turning error, 122

Bicyclist unexpected turn/swerve, 12, 120

Motorist drive-out, 123, 125, 126

Motorist drive-through, 123

Motorist intentionally caused, 126

Motorist lost control, 122

Motorist overtaking, 12, 64, 120, 124

Motorist turn/drive-out, 12, 120

Motorist turn/merge, 124

Motorist turning error, 122

Motorist unexpected turn, 121

Bicyclist crashes, 5, 12, 19, 41, 50, 57, 60, 64
Bittner, S.R., 19, 70, 104, 105, 106, 107, $127,129,135$

Blatt, J., 22, 41, 45, 67, 127, 137

Blomberg, R.D., 14, 16, 17, 18, 19, 20, 21, $22,24,25,27,28,31,40,41,42,53,57$, $60,63,64,65,68,69,71,72,73,75,76$, $77,78,79,80,81,82,83,84,87,89,90$, 91, 92, 93, 94, 98, 100, 101, 102, 103, $105,108,109,127,128,129,130,131$, 132, 133, 134, 135, 136, 137, 138, 139

Brown, R., 17, 44, 129, 134

Bus, 8, 14, 15, 17, 20, 21, 24, 29, 47, 54, 69, $70,71,73,79,80,84,88,104,105,106$, $107,113,117,124,129,130,138,139$

Bus stop, 14, 15, 17, 47, 69, 71, 79, 88, 113, 117

Casey, S.M., 18, 41, 42, 129, 135

CDC, 26

Center for Applied Research, 26, 62

Centers for Disease Control and Prevention, 26

Chen, M., 50, 131, 138

Children, 8, 14, 15, 16, 17, 19, 20, 21, 22, $24,25,35,38,42,45,54,57,58,69,71$, $72,73,76,77,78,87,96,97,98,99,100$, $101,102,103,104,105,106,107,113$, $116,126,127,128,129,132,134,136$, 137

Elementary school, 16, 19, 21, 69, 70, 104, 105, 106, 107, 129, 135, 138 Junior/senior high school, 21, 104, 105, $106,107,127,135$

Preschool, 19, 20, 35, 58, 96, 97, 99, 127, 132, 137

Chiplock, L.W., 19, 21, 24, 69, 70, 104, 105, $106,107,127,129,130,135$

Cleven, A.M., 20, 24, 25, 27, 28, 53, 65, 69, $89,90,91,92,93,94,105,108,109,127$, $128,129,138,139$

Colker, L., 20, 96, 97, 99, 132, 137

Collision point, 11

Compton, R.P., 24, 57, 60, 129, 138

Conspicuity, 13, 19, 20, 23, 24, 27, 35, 58, $63,64,65,82,83,108,128,130,137$

Cosgrove, M., 20, 96, 97, 99, 132, 137 
Countermeasure, $3,5,7,8,9,10,12,13,14$, $15,17,18,19,20,21,22,23,24,25,26$, $27,28,31,35,36,37,38,39,40,41,42$, $43,45,46,47,50,51,52,56,57,58,61$, $63,64,65,66,67,68,69,71,72,73,75$, $76,77,78,79,80,81,82,83,84,85,86$, $87,88,89,90,91,92,93,96,97,98,99$, $101,102,103,108,109,127,128,129$, $130,131,132,133,134,135,136,137$, 139

Countermeasure evaluation, 13, 47

Cox, C.L., 23

Crash data, 15, 22, 24, 37, 56, 68, 69, 72, 75, 76, 78, 80, 83, 97, 108

Crash generation process, 9, 10

Crespi. I., 22, 38, 133, 136

Critical actions, 9, 10

Cross, K.D., 3, 5, 9, 10, 11, 12, 13, 18, 19, $32,33,40,41,42,43,46,51,68,75,83$, $128,129,134,135,136$

Dart-out, 15

DeBartolo, K.B., 17, 19, 57, 60, 79, 84, 87, $129,132,135,136$

Decibel, 44

Dingus, T.A., 54, 130, 139

Disabled vehicle, 20, 21, 39, 73, 80, 82, 113, 116

Dismounted motorist, 20, 79, 80, 82, 84, 133,136

Diversity, 23

Drivers, 7, 8, 14, 21, 22, 24, 27, 29, 38, 49, $54,69,71,73,82,95$

Dueker, R.L., 16, 19, 21, 22, 24, 41, 45, 67, 69, 70, 99, 100, 101, 102, 103, 104, 105, $106,107,127,129,130,132,134,135$, 136,137

Dunlap and Associates, 26, 27, 51, 52, 53, 62, 130, 138, 139

Edwards, J.M., 21, 24, 65, 72, 73, 76, 77, 78, 108, 109, 128, 132, 137, 138

Ehrlich, P., 22, 58, 130, 136

Eisner, E.J., 15, 47, 132, 134

Enforcement, 14, 16, 23, 25, 27, 28, 31, 35, $49,54,58,61,79,84,86,87,93,94,129$, $132,133,135$

Engineering, 23, 25, 27, 31, 35, 49, 57, 58, $61,88,90,93,94,109$

Environmental factors, 9
Farina, A., 22, 58, 130, 136

Farrell, M.L., 18, 21, 40, 41, 68, 72, 73, 75, $76,77,78,83,128,132,136,137$

Federal Highway Administration, 1, 14, 15, $19,25,26,27,28,31,33,49,50,51,52$, $58,60,62,109,131,138$

Fell, J.C., 9, 22, 56, 130, 133, 135

FHWA, 1, 14, 15, 19, 25, 26, 27, 28, 31, 33, $49,50,51,52,58,60,62,109,131,138$

Fisher, G., 3, 5, 9, 10, 11, 12, 13, 19, 32, 33, $40,42,43,46,51,68,75,83,129,134$, 135

Fluorescence, 20, 64, 82

Function failures, 9, 10

Function/event sequence, 5, 6, 7

Fusees, 20, 82

Gaskins, C., 54, 130, 139

Gender, 50

Males, 23, 56, 90, 91, 92

Hale, A., 14, 17, 18, 19, 20, 22, 40, 41, 63, $64,65,68,71,72,73,75,76,77,78,79$, $80,81,83,84,87,89,90,91,92,98,101$, $102,103,128,130,133,135,136,137$

Hanowski, R.J., 29, 54, 130, 139

Harkey, D.L., 50, 131, 138

Heed the Speed, 28, 93, 94, 127, 139

Helmet, 35, 85, 133, 138

Hickman, J.S., 54, 130, 139

Highway Safety Research Center, 26, 50, $51,52,62$

Holbrook, T., 54, 130, 139

HSRC, 26, 50, 51, 52, 62

Huey, R., 54, 130, 139

Hunter, W.W., 23, 25, 31, 61, 67, 131, 132, 137,138

Injury, 23, 25, 49, 50, 53, 56, 59, 89, 90, 93, 101, 102, 103, 109, 131, 133, 138

Institute of Transportation Engineers, 26

Intersection, 8, 12, 14, 15, 19, 21, 24, 36, 38, $39,47,50,56,57,69,71,73,77,79,88$, $104,105,106,107,108,114,116,118$, $120,121,123,124,125,126$

ITE, 26

Kane, J.N., 131, 136

Kearney, E.F., 17, 20, 79, 80, 81, 84, 87, 98, $128,130,133,135,137$ 
Knoblauch, R.L., 3, 5, 7, 8, 9, 11, 13, 14, 15, $16,19,20,31,32,33,36,37,39,43,45$, $46,73,80,131,132,133,134$

Krull, K.A., 50, 131, 138

Laws, 18, 19, 23, 24, 27, 38, 49, 53, 60, 61, 69, 81, 83, 86, 128, 131, 133, 139

Laws, F.D., 9, 131, 133

Leaf, W.A., 18, 19, 20, 21, 22, 23, 33, 40, $41,42,57,60,68,71,72,73,75,76,77$, $78,79,81,82,83,84,89,90,91,92,93$, $101,102,103,128,129,131,132,133$, $135,136,137,138$

Left turn on red, 24, 57, 60

Left-right-left, 16

Levine, J.M., 15, 47, 132, 134

Llaneras, E.E., 54, 130, 139

LTOR, 24

Lund, A.K., 22, 72, 73, 76, 77, 78, 132, 137

Mail box, 21, 73

McGlade, F., 9, 131, 133

Mekemson, J., 50, 131, 138

Messages, 16, 17, 18, 22, 24, 35, 40, 49, 58, $68,71,72,73,74,75,76,77,78,83,100$, 101, 102, 103, 108, 128, 132, 134, 136, 137

Miami-Dade Project, 28

MICTO, 17

Midblock, 8, 12, 16

Miller, S., 54, 130, 139

Milton, E.V., 24, 57, 60, 129, 138

Model Ice Cream Truck Ordinance, 17, 98

Model regulations, 14, 16, 17, 20, 21, 41, $58,68,75,79,80,81,82,83,84,87,98$, $128,130,133,135,136$

Moore, Jr, W, 20, 36, 39, 80, 131, 134

Motorist unexpected left turn, 12

National Association for the Education of Young Children, 20, 97

National Center for Statistics and Analysis, 1

National Committee on Uniform Traffic Laws and Ordinances, 27, 53

National Highway Traffic Safety Administration, iii, 1, 2, 9, 13, 14, 15, 16, $17,18,19,20,23,24,25,26,27,28,29$, $31,33,35,36,37,39,40,42,45,49,50$, $51,52,54,57,58,59,60,61,62,63,65$, $66,67,68,69,71,72,73,75,76,77,78$,
$79,80,81,82,83,84,87,89,90,91,92$, 93, 95, 96, 97, 98, 99, 100, 101, 102, 103, $104,105,106,107,108,109,129,130$, $131,132,133,134,138,139$

National Safety Council, 20, 24, 25, 49, 69, 105

National Technical Information Service, 1, 2 NCSA, 1

NCUTLO, 27, 53

Neighborhoods, 28, 94

New technology/tools, 35, 45, 47, 49, 50, $51,52,53,54$

NHTSA, iii, 1, 2, 5, 9, 13, 14, 15, 16, 17, 18, $19,20,23,24,25,26,27,28,29,31,33$, $35,36,37,39,40,42,45,49,50,51,52$, $54,57,58,59,60,61,62,63,65,66,67$, $68,69,71,72,73,75,76,77,78,79,80$, $81,82,83,84,87,89,90,91,92,93,95$, $96,97,98,99,100,101,102,103,104$, $105,106,107,108,109,129,130,131$, $132,133,134,138,139$

Non-intersection, 56, 118, 125, 126

NTIS, 1, 2

Older adult, 21, 24, 35, 58, 65, 73, 108, 109, $127,128,138$

Olson, R.L., 54, 130, 139

Operator factors, 9

PAK, 22, 45

Parental supervision, 99

Parents, 20, 21, 24, 58, 68, 69, 71, 73, 97, 99, 105

Parking, 8, 15, 16, 17, 21, 38, 44, 77, 79, 81, $83,87,88,114,116,119,121,124$

Partnership for a Walkable America, 26, 62, 132

Pavlinski, L., 22, 58, 130, 136

PBCAT, 23, 26, 34, 50, 111, 122, 131, 138

Pedestrian and Bicycle Information Center, 26, 62

Pedestrian crash type

Backing vehicle, 8, 17, 21, 24, 44, 47, 69, 73, 77, 79, 97, 108, 113, 116, 120, 126

Bus stop related, 14, 15, 17, 47, 69, 71, 79, 88, 113, 117

Commercial bus related, 8, 113, 117

Dart-out, 8, 15, 16, 21, 22, 36, 38, 39, 47, $69,71,72,73,76,79,80,81,82,87$, 
$88,96,97,99,100,101,102,103,104$, $105,106,107,118,129,130,135,136$

Disabled vehicle, 20, 21, 39, 73, 80, 82, 113, 116

Driverless vehicle, 113, 116

Emergency vehicle-related, 113, 116

Exiting/entering parked vehicle, 113, 117

Failed to yield, 118

Freeway crossing, 47

Intersection dash, 8, 21, 36, 38, 39, 47, $69,71,73,79,88,104,105,106,107$, 114

Mailbox related, 80, 104, 105, 106, 107, 113, 117

Multiple threat, 8, 15, 21, 22, 36, 47, 69, $71,72,76,78,79,81,88,104,105$, $106,107,114,115,118,123$

Off roadway, 119

Playing in roadway, 99, 113, 116

Play-vehicle related, 99, 113, 116, 126

School bus related, 17, 20, 21, 24, 29, 54, $69,70,73,80,84,104,105,106,107$, $113,117,129,130,138,139$

Trapped, 114, 118, 123

Vehicle turn/merge, 8, 22, 24, 36, 47, 71, $72,76,78,88,108,114$

Vendor/ice cream truck, $8,15,17,38,47$, $71,79,88,98,113$

Waiting to cross, 114,117

Walking along roadway, 64, 104, 105, 106, 107, 117

Working in road, 47, 116

Pedestrian crashes, $7,11,12,13,14,15,16$, $17,20,21,22,23,25,27,35,36,37,38$, $39,43,44,46,47,49,50,53,56,57,58$, $59,66,67,71,72,73,76,77,78,79,80$, $81,84,86,89,90,91,92,93,96,98,99$, $104,105,106,107,109,127,131,132$, $137,138,139$

Pedestrian fatalities, 14, 58, 59, 90, 108

Pedestrian injuries, 14

PedSafe, 19, 21, 70, 104, 105, 106, 107, $127,129,135$

Pein, W.E., 23, 131, 138

Perchonok, K., 9, 132, 134

Phinney, J., 20, 96, 97, 99, 132, 137

PI\&E, 14, 15, 16, 17, 18, 21, 22, 24, 25, 28, $31,40,68,72,75,76,78,80,83,84$
Precipitating factors, $7,10,26,36,37,39$, 43, 45, 73, 82, 132, 133

Predisposing factors, $7,10,14,19,37,39$, 99

Preparatory phase, 11

Preusser, C.W., 72, 73, 76, 77, 78, 132, 137

Preusser, D.F., 14, 16, 17, 18, 19, 21, 22, 23, 24, 33, 57, 60, 63, 64, 71, 72, 73, 76, 77, $78,79,84,87,89,90,91,92,93,98,100$, $101,102,103,128,129,130,131,132$, $133,134,135,136,137,138$

Problem identification, 13, 14, 19, 23, 35, $36,37,38,39,40,42,43$

Program assessment kit, 22, 45

Program review, 25, 35, 56, 57, 58, 59, 60, 61

Public information and education, 72, 75, $76,78,80,84$

Racial/ethnic groups, 23, 89, 90, 91, 92

Radio, 18, 21, 22, 73, 75

Reactive phase, 11

Regulations, 14, 15, 16, 17, 20, 21, 24, 35, $38,41,49,58,68,69,75,79,80,81,82$, $83,84,86,87,98,128,130,131,132$, $133,135,136$

Regulatory countermeasures, 14, 17

Relative risk due to alcohol, 14

Resource guide, 26, 27, 28, 51, 52, 53, 85, $128,130,133,138,139$

Right turn on red, 19, 24, 57, 60, 120, 123, $129,132,136,138$

Road worker, 20, 79, 80, 82

Robert Wood Johnson Foundation, 26

Rose, A.M., 15, 47, 132, 134

Ross, H.L., 9, 127, 133

RTOR, 19, 24

Rural areas, 13, 19, 20, 21, 24, 35, 36, 39, $46,56,69,70,72,73,76,77,78,79,80$, $84,104,105,106,107,127,129,130$, $131,132,134,135,137$

Safe Routes to School, 34

Safety messages, 16, 17, 18, 22, 24, 49, 58, $71,72,73,76,77,78,100,101,102,103$, $108,128,132,134,137$

Schaudt, W.A., 54, 130, 139

Schmitz, P.R., 20, 36, 39, 80, 131, 134

School age children, 35, 98, 99, 100, 101, 102, 103, 104, 105, 106, 107 
School bus, 17, 20, 21, 24, 29, 54, 69, 70, $73,80,84,104,105,106,107,113,117$, $129,130,138,139$

Search, 6, 7, 14, 15, 16, 18, 19, 23, 33, 35, $36,37,39,41,42,45,47,51,52,56,57$, $60,63,65,66,67,68,69,71,72,73,75$, $77,78,79,80,81,84,87,89,90,91,92$, 93, 95, 96, 97, 98, 99, 100, 101, 102, 103, 104, 105, 106, 107, 108, 109, 129, 135

Shapiro, R.G., 84, 130, 137

Singer, S., 16, 86, 132, 133

Snyder, M.B., 3, 5, 7, 8, 9, 11, 13, 14, 15, $16,19,31,32,33,36,37,39,43,45,73$, 132,133

Sommers, B.J., 36, 39, 131, 134

Spanish, 22, 78

Spaulding, J.M., 54, 130, 139

Speed, 20, 23, 27, 28, 35, 47, 56, 82, 88, 93, $94,95,98,122,131,138$

SRTS, 34

Stop signs, 93, 120

STOP swing arm, 17

Stutts, J.C., 23, 25, 61, 67, 131, 132, 137, 138

Suburban areas, 13, 19, 20, 21, 24, 35, 36, $39,46,69,70,79,80,81,104,105,106$, $107,130,131,134,135$

Supervision, 19, 21, 73, 99, 132, 136

Sutherland, L.C., 17, 44, 129, 134

Swing arm, 20, 98

Synthesis, 35, 56, 57, 58, 59, 60, 61

Tarrants, W.E., 22, 58, 130, 136

Television, 18, 21, 22, 48, 73, 75, 100
Terminal event, 9, 10

Thackray, Jr. R.M., 19, 99, 132, 136

Toth, G.R., 22, 56, 130, 135

Tracy, L., 25, 61, 67, 85, 132, 133, 137, 138

Traffic engineering, $15,49,57,58$

Training, 16, 19, 20, 21, 24, 31, 35, 40, 66, $67,68,69,70,75,83,100,101,102,103$, $104,105,106,107,127,128,129,130$, $134,135,136,138$

Ulmer, R.G., 14, 20, 79, 82, 84, 89, 90, 91, $92,128,133,135,136$

Urban areas, 13, 15, 24, 35, 36, 37, 38, 39, $46,58,67,69,72,73,76,77,78,80,86$, $88,98,99,100,101,102,103,127,131$, $132,134,137$

Vayda, A., 22, 38, 133, 136

Vehicle factors, 9

Vehicle turn/merge, 8, 22, 24, 36, 47, 71, 72, $76,78,88,108,114$

Video, 24, 54, 69, 109

Virginia Tech Transportation Institute, 29, 54

Visibility, 19, 35, 58, 63, 64

Walk Alert, 25, 49, 132, 138

Walk to School Day, 26, 62

Warning device, 44, 82, 129, 134

WESTAT, 29, 54

Wilkinson, W.C., 25, 61, 67, 132, 137

Zeidler, P, 19, 63, 65, 130, 137

Zone Guide, 25

Zones, 25, 27, 44, 65, 91, 108, 109, 127, 138

Zylman, R., 14, 89, 90, 133 

DOT HS 810793

July 2007 